\title{
SCHRÖDINGER SEMIGROUPS
}

\author{
BY BARRY SIMON
}

\begin{abstract}
Let $H=-\frac{1}{2} \Delta+V$ be a general Schrödinger operator on $R^{\nu}$ $(\nu \geqslant 1)$, where $\Delta$ is the Laplace differential operator and $V$ is a potential function on which we assume minimal hypotheses of growth and regularity, and in particular allow $V$ which are unbounded below. We give a general survey of the properties of $e^{-t H}, t>0$, and related mappings given in terms of solutions of initial value problems for the differential equation $d u / d t+$ $H u=0$. Among the subjects treated are $L^{p}$-properties of these maps, existence of continuous integral kernels for them, and regularity properties of eigenfunctions, including Harnack's inequality.
\end{abstract}

\section{CONTENTS}

A. Introduction
A1. Overview

A2. The class $K_{\nu}$

A3. Literature on larger classes

B. $L^{p}$-properties

B1. $L^{P}$-smoothing of semigroups

B2. Sobolev estimates

B3. Continuity and derivative estimates

B4. Localization

B5. Growth of $L^{p}$-semigroup norms as $t \rightarrow \infty$

B6. Weighted $L^{2}$-spaces

B7. Integral kernels: General potentials

B8. Integral kernels: Some special operators for some special potentials

B9. Trace ideal properties

B10. Continuity in $V$

B11. Hypercontractive semigroups and all that

B12. Some remarks on the case when $H$ is unbounded below

B13. The magnetic case

C. Eigenfunctions

C1. Harnack's inequality and subsolution estimates

C2. Local estimates on $\nabla \varphi$

C3. Decay of eigenfunctions

C4. Eigenfunctions and spectrum

C5. Eigenfunction expansions

Received by the editors March 4, 1982.

1980 Mathematics Subject Classification. Primary 81-02, 35-02; Secondary 47F05, 35P05.

() 1982 American Mathematical Society 0273-0979/82/0000-0350/821.00 
C6. The local spectral density and its classical limit

C7. The integrated density of states

C8. Allegretto-Piepenbrink theory

C9. Unique continuation

\section{A. INTRODUCTION}

A1. Overview. By a Schrödinger operator, we mean a partial differential operator on $R^{\nu}$ of the form

$$
H=H_{0}+V ; \quad H_{0}=-\frac{1}{2} \Delta ; \quad V=V(x)
$$

where $\Delta$ is the $\nu$-dimensional Laplace operator $\Delta=\sum_{j=1}^{\nu} \partial^{2} / \partial x_{j}^{2}$ (the reason for the convention $-\frac{1}{2} \Delta$ rather than $-\Delta$ will become clear later). The name comes from the form of Schrödinger's equation which, in units with $\mathbf{h}=m=1$ reads

$$
i \partial \psi / \partial t=H \psi \text {. }
$$

$H$ is thus the Hamiltonian operator of a nonrelativistic particle; $H_{0}$ is the kinetic energy and $V$ the potential energy. The function, $V$, will not be supposed smooth, continuous or even bounded, and indeed the Coulomb potentials present in atomic and molecular Hamiltonians are unbounded as one approaches certain codimension 3 planes. One of our goals will be to discuss fairly general $V$ 's.

In $\S \mathrm{B} 13$ we will discuss operators of the form

$$
H=\frac{1}{2}(-i \nabla-a)^{2}+V
$$

which describes the Hamiltonian in the presence of a potential, $V$, and magnetic field $B=\nabla \times a$.

By a Schrödinger semigroup, we mean the semigroup

$$
e^{-t H}
$$

generated by $H$. Our purpose in this article is to reveiw a variety of properties of these operators, especially within and between $L^{p}$-spaces. The symbol $\|A\|_{p, q}$ denotes the norm of an operator $A$ from $L^{p}$ to $L^{q}$. This appears to be a narrow and probably uninteresting subject. In fact, it impinges on a considerable number of different aspects of the study of $H$ and on the general theory of semigroups, of elliptic equations and of stochastic processes.

(1) There occur examples of interest to the general theory of semigroups. For instance, we will see (§B5) that for suitable $V$ in $C_{0}^{\infty}\left(R^{4}\right)$, the semigroup $e^{-t H}$ on $L^{\infty}\left(R^{4}\right)$ has an operator norm behaving as $C t / \ln t$ as $t \rightarrow \infty$.

(2) At first sight, it appears that (A3) is the wrong operator to look at; the Schrödinger equation (A2) is solved by $\psi(t)=e^{-i t H} \psi(0)$, so that for quantum mechanics the correct object to look at is the unitary group

$$
e^{-i t H} \text {. }
$$

While this is to some extent true, one is especially interested in eigenfunctions $H \psi=E \psi ; \psi \in L^{2}$ (called stationary states, since then $(\psi(t), A \psi(t))=(\psi, A \psi)$ so that the probability distributions of observables is time-independent). Of course, such an eigenfunction obeys $e^{-t H} \psi=e^{-t E} \psi$ so $\psi$ is in $e^{-t H}\left[L^{2}\right]$. Thus, for example, results that say $e^{-t H}$ maps $L^{2}$ to $L^{\infty}$ ensure us that eigenfunctions 
are in $L^{\infty}$ (since $V$ may be unbounded, this is not trivial). Moreover, $e^{-i t H}\left[L^{2}\right]$ is all of $L^{2}$ so $e^{-i t H}$ cannot be used for this purpose. In fact, a major element (Chapter C) of our study here will be to study eigenfunctions.

(3) The Schrödinger semigroup is useful for studying any eigenfunction but particularly so for eigenfunctions at the bottom of the spectrum. The quantity inf $\sigma(H)$ is called the ground state energy and if it is an eigenvalue, the corresponding eigenvector is called the ground state. The ground state is always unique if it exists, i.e. if the ground state energy is an eigenvalue, it has multiplicity one. Indeed, this is most easily proven using the semigroup (see e.g. [162, §XIII.12]); explicitly, the fact that $e^{-t H} f$ is pointwise positive if $f$ is pointwise nonnegative. The ground state energy is given by

$$
\text { inf } \sigma(H)=-\lim _{t \rightarrow \infty} t^{-1} \ln \left\|e^{-t H}\right\| \text {. }
$$

In (A5), the norm is for an operator on $L^{2}$, but we will see later (§B5) that one can use an $L^{p}$-operator norm instead. An alternative formula is

$$
\text { inf } \sigma(H)=-\lim _{t \rightarrow \infty} t^{-1} \ln \left(f, e^{-t H} f\right)
$$

for any $f$ which is pointwise nonnegative. Finally, if $H$ has a ground state $\psi$, then

$$
\psi=s-\lim _{t \rightarrow \infty} e^{-t H} f /\left(f, e^{-2 t H} f\right)^{1 / 2}
$$

where again, $f$ is any nonnegative function.

(A7) is particularly important in quantum field theory, where the use of semigroups has become a standard tool. Because relativistic invariance becomes Euclidean invariance if $t i$ is replaced by $t$, the associated theory is called Euclidean quantum field theory. These ideas are especially powerful in a path integration context; see (8) below. For further discussion of these things, see $[76,193,204]$.

(4) Sobolev estimates are among the most celebrated and useful estimates in analysis. Since $\Delta f, f \in L^{p}$, if and only if $f \in[-\Delta+1]^{-1}\left[L^{p}\right]$, the Sobolev estimates for $-\Delta$ are statements about the resolvent of $-\Delta$ mapping $L^{p}$ to $L^{q}$. Analogous estimates for $H$ will be proven here $(\S \mathrm{B} 2)$ by using the semigroup $L^{p}$ estimates and the formula

$$
(H+E)^{-1}=\int_{0}^{\infty} e^{-t H} e^{-t E} d t
$$

The limitation on $q$ will come from the divergence of $\left\|e^{-t H}\right\|_{p ; q}$ as $t \downarrow 0$. We can also study $(H+E)^{-\alpha}$ by

$$
(H+E)^{-\alpha}=c_{\alpha} \int_{0}^{\infty} e^{-t H} e^{-t E} t^{\alpha-1} d t
$$

so long as $\alpha>0$. [ $c_{\alpha}$ is a constant expressible as a $\Gamma$ function.] The net result will be that we recover "inhomogeneous" but not "homogeneous" Sobolev estimates; i.e. $(-\Delta+1)^{-\alpha}$ maps $L^{p}$ to $L^{q}$ if $p \leqslant q \leqslant f(\alpha, p)$ for an explicit function $f$. We show $(H+E)^{-\alpha}$ maps $L^{p}$ to $L^{q}$ if $p \leqslant q<f(\alpha, p)$ (i.e. we lose the case $q=f(\alpha, p))$ and if $E$ is sufficiently large. 
(5) It is a comforting fact to learn that some operator one is interested in has an integral kernel. This is especially beloved by physicists, in part because the asymptotics of this kernel at infinity are sometimes related to physically significant quantities like scattering amplitudes. The theorem of Dunford and Pettis (see Trèves [202]) asserts that

THEOREM A.1.1. Let $(M, \mu)$ be a separable measure space and $E$ a separable Banach space. Let $A$ be a bounded operator from $E$ to $L^{\infty}(M, d \mu)$. Then there exists a unique (up to sets of $\mu$-measure zero) weakly measurable function, $a$, from $M$ to $E^{*}$ so that for each $f \in E$ and a.e. $x \in M$

$$
(A f)(x)=\langle a(x), f\rangle \text {. }
$$

Moreover $\|a\|_{\infty}=\|A\|$.

In particular, choosing $E=L^{p}(M, d \mu) ; 1 \leqslant p<\infty$, so that $E^{*}=$ $L^{q}(M, d \mu)$ with $q^{-1}+p^{-1}=1$, and noting the trivial converse of Theorem A.1.1, we have

COROLlARY A.1.2. If $A$ is a bounded operator on $L^{p}(M, d \mu)$ and $A$ is bounded also from $L^{p}$ to $L^{\infty}$ then there is a measurable function $a$ on $M \times M$ obeying

$$
\sup _{x}\left[\int|a(x, y)|^{q}\right]^{1 / q}\left(=\|A\|_{p ; \infty}\right)<\infty
$$

so that, for any $f \in L^{p}$,

$$
(A f)(x)=\int a(x, y) f(y) d \mu(y) .
$$

Conversely, if $A: L^{p} \rightarrow L^{p}$ has an internal kernel, $a$, in the sense of (A11) obeying (A10), then $A$ is a bounded map from $L^{p}$ to $L^{\infty}$.

Of especial interest is the case $p=2$. Operators on $L^{2}$ obeying (A10) are called Carleman (integral) operators and are of classical interest. Corollary A.1.2 is often attributed to Korotkov [120] especially in the Russian literature. Although it is a special case of the much older theorem of Dunford and Pettis [202], Korotkov was the first to emphasize its usefulness in the context of Carleman operators.

One can ask about continuity of the integral kernels. Let $X$ be a separable locally compact metric space and let $C(X), \mathfrak{N}_{f}(X)$ denote the bounded continuous functions on $X$ and its dual, the Baire measures of finite total variation. By $\sigma(C, \mathfrak{T})$ we mean the weak topology on $C$ determined by the functions $f \rightarrow \int f d \mu$ and by $\sigma(\Re, C)$ the weak * topology on $\Re$. Given any Baire measure, $\nu$, finite or not, on $X, L^{1}(X, d \nu)$ is imbedded in $\Re$ by associating $f$ to $f d \nu$ and if $\operatorname{supp} \nu=X$, then $L^{1}$ is $\sigma(\Re, C)$ dense. One can prove quite easily that

TheOREM A.1.3. Let $X$ be a separable locally compact space and $\nu$ a Baire measure. Let $T$ be bounded map from $L^{1}(X, d \nu)$ to $L^{\infty}(X, d \nu)$. Then $T$ has an integral kernel which is separately continuous in $x, y$ if and only if $\operatorname{Ran} T \subset C(X)$ and $T$ has an extension $\tilde{T}$ from $\mathfrak{T}(X)$ to $C(X)$ which is continuous in norm and also when $\Re$ is given the $\sigma(\mathfrak{N}, C)$ topology and $C$ the $\sigma(C, \mathfrak{T})$ topology. If $\operatorname{supp} \nu=X$, then such $a \tilde{T}$ is unique. 
The integral kernel is given by $K(x, y)=\tilde{T}\left(\delta_{y}\right)(x)$ with $\delta_{y}$ the point measure at $y$.

We will not use the above theorem in this paper because it only yields separate continuity in $x, y$; by other methods, we will be able to prove that the various operators of interest have jointly continuous kernels. It does not seem easy to get such joint continuity from Banach space continuity properties of $T$.

(6) Carleman operators are of especial interest because it is precisely for them that there exist eigenfunction expansions of the type associated with the names of Berezanskii, Browder, Garding, Gel'fand and Kac (we dub this a BGK expansion). In §C5, we discuss such expansions and compare them with the more powerful expansion of Povzner and Ikebe (IP expansions). The BGK expansions are of minimal interest in the study of " $N$-body potentials" (see A2) where one knows or expects there is an IP expansion. But with the recent interest in random (e.g. [148]) and almost periodic potentials (e.g. [192]), general results which hold for potentials with no special decay properties at infinity have become very significant. In this article we primarily emphasize results that hold for potentials without any decay assumptions. The BGK expansions are exactly of this type and have played an important role in the study of random and almost periodic potentials.

(7) Properties of integral kernels are important in establishing trace ideal [195] properties of operators of the form $f(H) g(x)$. Such properties are often technically very useful and are discussed in §B9.

(8) Thus far we have emphasized connections of the subject of this article with functional analysis and partial differential equations. There is also a deep and powerful connection with the theory of stochastic processes, especially Brownian motion and certain Markovian perturbations of it. In fact, the proofs we sketch in $\S \S \mathrm{B} 1$ and $\mathrm{Cl}$ of the basic results whose consequence we derive later in the corresponding chapters, are probabilistic in nature. We emphasize that most of the proofs of consequences are not probabilistic. Moreover, as we will discuss (and briefly describe for the results of B1), there are nonprobabilistic proofs of these results. We prefer the probabilistic proofs because we find them natural and moreover, because they set up a close connection between $L^{p}$ bounds on semigroups and $L^{p}$ bounds on the Poisson kernel for $H$, the key object in $\S \mathrm{Cl}$. From an analytic point of view, this connection is far from transparent and indeed the analytic proofs seem to be totally unrelated. But, in fairness, I should emphasize that I know experts in PDE's who regard the probabilistic proofs as highly unnatural: Chaqu'un son gout! We emphasize that because of the probabilistic proofs we are restricted to second order equations. Occasionally, in this article, we will suppose a familiarity with Brownian motion and the Feynman-Kac formula; see [194] for the necessary background.

This completes our overview of reasons why $L^{p}$ properties of semigroups are interesting. Other than the indications above, we will not bother to sketch the contents of Chapters B and C; the table of contents lists section titles which give considerable information.

We have already indicated that it is important to prove results without any decay assumptions on $V$ and obviously one wants to allow local singularities to be able to include Coulomb potentials. Virtually all our results will be stated 
for potentials in a class, $K_{\nu}$, discussed in $\S \mathrm{A} 2$; to be more precise, we will normally require that $V_{-} \equiv \max (-V, 0)$ lie in $K_{\nu}$ but that $V_{+} \equiv \max (V, 0)$ only lies in $K_{\nu}^{\text {loc }}$. In a sense we describe in $\S \mathrm{A} 2, K_{\nu}$ is a maximal space for $e^{-t H}$ to have reasonable $L^{\infty} \rightarrow L^{\infty}$ properties, at least if $V$ doesn't have severe oscillations. Nevertheless, there are a few papers on larger classes and these are described in §A3.

We should emphasize that while we are careful to give our results for this big class, $K_{\nu}$, it is my opinion, that for most cases one could be quite happy with results that included Coulomb singularities and all continuous functions. The only legitimate point of studying larger classes is naturally of results or methods.

When $V$ is unbounded, the definition of the sum $-\Delta+V$ is not completely trivial. We briefly discuss the definition of $H$ if $V_{-} \in K_{\nu}, V_{+} \in K_{\nu}^{\text {loc }}$ in $\S \mathrm{A} 2$.

While we have emphasized that our results generally require no decay hypotheses on $V$, many are interesting even for $V$ 's with some decay, like $N$-body potentials. Of particular interest are results on properties of eigenfunctions and on certain operators having integral kernels.

There is one severe defect in the breadth of our class of potentials. Because of our central use of $e^{-t H}$, we cannot directly treat the case where $H$ is not bounded from below (except for the local results of $\S \mathrm{Cl}$ which are still applicable). One can reasonably define $H$ as long as $H+c|x|^{2}$ is bounded below for some $c$ even if $H$ is not bounded below. The important case of constant electric field, i.e. $V=W+\vec{a} \cdot \vec{x}$ with $\vec{a}$ fixed and $W$ in $K_{\nu}$ is in this class. In $\S B 12$ we will prove such operators have BGK eigenfunction expansions.

Having mentioned my book [194], I should deal with the question of overlap with that book and this article. $\$ 25$ of that book deals with the subject matter of $\S \S B 1$, B2, B7 and B9 of this paper. While most of [194] involves well developed subjects and therefore is to some extent in definitive form, $\$ 25$ represents results obtained in late 1977 when the book was being completed. There have been considerable developments since then, and this article subsumes and improves most of $\$ 25$ of [194].

While the present article is of "review" nature in the sense of giving a comprehensive overview of a subject, there are numerous new results. We will not generally bother to note those cases where the result stated here are proven for $K_{\nu}$ while previous results are for smaller classes. We will indicate results which are "substantially new"; many in the area of integral kernels of various operators.

The results and worldview of this paper have been developed during the past five years, and I owe a debt to many colleagues for useful discussions, suggestions and arguments during that period. I would like to thank them all, including S. Agmon, M. Aizenman, A. Berthier, H. Brezis, R. Carmona, E. B. Davies, P. Deift, I. Herbst, M. Hoffman-Ostenhof, T. Hoffman-Ostenhof, M. Klaus, E. Lieb, T. Osborne, J. Piepenbrink, M. Perelmuter, Y. Semenov, I. Sigal, B. Souillard and S. Zelditch. 
A2. The class $K_{\nu}$. The basic spaces of potentials we will consider are given by

Definition. A real-valued measurable function $V$ on $R^{\nu}$ is said to lie in $K_{\nu}$ if and only if

(a) If $\nu \geqslant 3$

$$
\lim _{\alpha \downarrow 0}\left[\sup _{x} \int_{|x-y| \leqslant \alpha}|x-y|^{-(\nu-2)}|V(y)| d^{\nu} y\right]=0 .
$$

(b) If $\nu=2$

$$
\lim _{\alpha \downarrow 0}\left[\sup _{x} \int_{|x-y|<\alpha} \ln \left\{|x-y|^{-1}\right\}|V(y)| d^{2} y\right]=0 .
$$

(c) If $\nu=1$

$$
\sup _{x} \int_{|x-y| \leqslant 1}|V(y)| d y<\infty
$$

We say $V$ is in $K_{\nu}^{\text {loc }}$ if and only if $V \mathscr{X}_{R} \in K^{\nu}$ for all $R$, where $\mathcal{X}_{R}$ is the characteristic function of $\{x|| x \mid \leqslant R\}$.

The earliest discussion of classes related to $K_{\nu}$ is that of Stummel [200] who defined the space $M_{\beta, 2}$ where $M_{\beta, p}$ is given by

Definition (SCHECHTER [172]). $V \in M_{\beta, p}$ if and only if

$$
\sup _{x} \int_{|x-y| \leqslant 1}|x-y|^{\beta-\nu}|V(y)|^{p} d^{\nu} y \equiv\|V\|_{\beta, p}^{p}<\infty .
$$

If $\nu \geqslant 3$, we will put the norm $\|\cdot\|_{2,1}$ on $K_{\nu}$ (in which $K_{\nu}$ is not a complete space) when we discuss continuity in $V$ in $\S \mathrm{B} 10$. For $\nu=2$, we use the norm

$$
\sup _{x} \int_{|x-y| \leqslant 1 / 2} \ln \left\{|x-y|^{-1}\right\}|V(y)| d^{\nu} y
$$

and in $\nu=1$, the uniform $L^{1}$ norm, i.e. $\|\cdot\|_{1,1}$. In all these cases, we use the symbol $\|\cdot\|_{k}$ for this norm. As we shall see, if $\nu \geqslant 3, K_{\nu}$ is not complete in this norm.

In his book, Schechter [172] does not explicitly single out the space $K_{\nu}$ but if one chases through several definitions, one discovers that one of his theorems has as its hypothesis $V \in K_{\nu}$. The classes appear explicitly first in Kato's paper [112]. Ironically, in the context of the problems studied by Schechter and Kato (form sum and selfadjointness questions respectively), $K_{\nu}$ is not the natural class; e.g. if $\nu \geqslant 3$ and $V(x)=-|x|^{-2}\{\ln (|x|+2)\}^{-\alpha}$, then $V \in K_{\nu}$ if and only if $\alpha>1$ (see Example B below), but $V$ is $-\Delta$-form bounded with relative bound zero so long as $\nu \geqslant 3, \alpha>0$ (and $-\Delta$-operator is bounded if $\nu \geqslant 5, \alpha>0$ ). Schechter [170] subsequently realized that for form problems, the maximal classes are larger than $K_{\nu}$. [In $\S \mathrm{B} 1$, we will mention one explanation of why, in Kato's proof of selfadjointness, $K_{\nu}$ enters naturally; that is, while $K_{\nu}$ is not maximal for the selfadjointness, it is for one proof.]

The naturalness of the class $K_{\nu}$ for $L^{p}$ properties was discovered by Aizenman and Simon [9] in the context of path integral methods, and by 
Agmon [3] using PDE methods. This is illustrated by the following result which we will prove in $\S \mathrm{B} 1$.

TheOREM A.2.1 [9]. Let $V \leqslant 0$. Then $V \in K_{\nu}$ if and only if $e^{-t H}$ is bounded from $L^{\infty}$ to $L^{\infty}$ with $\lim _{t \downarrow 0}\left\|e^{-t H}\right\|_{\infty, \infty}=1$.

If $V$ has both signs, cancellations can take place for highly oscillatory potentials [9].

Aizenman and Simon also show that $V \in K_{\nu}^{\text {loc }}$ is equivalent when $V \leqslant 0$ to a strong version of Harnak's inequality [9, Theorems 5.1 and 5.2].

We should point out one interesting aspect of Theorem A.2.1. If $L^{\infty}$ to $L^{\infty}$ is replaced by $L^{2}$ to $L^{2}$, then the condition $\lim _{t \downarrow 0}\left\|e^{-t H}\right\|_{2,2}=1$ follows by the spectral theorem once $e^{-t H}$ is bounded. Thus the analogous question is whether $V$ is bounded below. In the $L^{2}$ case, one has the phenomena that there are potentials (like $-r^{-2}$ ) so that $V$ is in the class but $\lambda V$ is not for $\lambda$ large. Since $K_{\nu}$ is a vector space, we have the remarkable fact that if $V \leqslant 0$, then once $e^{-t\left(H_{0}+V\right)}$ is bounded on $L^{\infty}$ with the norm condition, automatically, the same is true for $e^{-t\left(H_{0}+\lambda V\right)}$ for all $\lambda$.

The function $|x-y|^{-(\nu-2)}$ arises in the definition of $K_{\nu}$ because it is (up to a constant) the integral kernel of $(-\Delta)^{-1}$. More generally, the integral kernel $G_{0}(x-y ; E)$ of $(-\Delta+E)^{-1}$ obeys (if $\nu \geqslant 3 ; E>0$ )

(a) $G_{0}(x-y ; E) \leqslant c_{\nu}|x-y|^{-(\nu-2)}$.

(b) For each $r>0$,

$$
\lim _{E \rightarrow \infty} \sup _{|x-y| \geqslant r} e^{|x-y|} G_{0}(x-y ; E)=0 .
$$

From these facts and the elementary

LEMMA A.2.2. $K_{\nu} \subset L_{\text {unif,loc }}^{1} \equiv\left\{V\left|\sup _{x} \int_{|x-y|<1}\right| V(y) \mid d^{\nu} y<\infty\right\}$.

One obtains the result (i) $\Rightarrow$ (ii) in the following

Proposition A.2.3 [9]. The following are equivalent:

(i) $V \in K_{\nu}$.

(ii) $\lim _{E \rightarrow \infty}\left\|(-\Delta+E)^{-1}|V|\right\|_{\infty}=0$.

(iii) $\lim _{E \rightarrow \infty}\left\|(-\Delta+E)^{-1} V\right\|_{\infty, \infty}=0$.

(iv) $\lim _{E \rightarrow \infty}\left\|V(-\Delta+E)^{-1}\right\|_{1,1}^{\infty}=0$.

(v) For all $\varepsilon>0$, there exist $a>0$ so that for all $\psi$ in $D_{L^{1}}(\Delta)$ (the domain of the generators of the semigroup $e^{\Delta t}$ on $L^{1}$ ), we have

$$
\|V \psi\|_{1} \leqslant \varepsilon\left\|H_{0} \psi\right\|_{1}+b\|\psi\|_{1} .
$$

In (ii), we intend the operator $(-\Delta+E)^{-1}$ applied to the function $|V|$ and take the $L^{\infty}$-norm. In (iii), (iv), $V$ is intended as a multiplication operator and we use an operator norm.

SKetch ( $\nu \geqslant 3$; THE PROOF IN $\nu=1,2$ IS SIMILAR). We have shown (i) $\Rightarrow$ (ii) and (ii) $\Rightarrow$ (i) is similar using lower bounds on $G_{0}$. Since $A=(-\Delta+E)^{-1}|V|$ takes positive functions to positive functions, we have that $|A f| \leqslant\|f\|_{\infty}$ (A1) pointwise ( 1 is the function which is identically 1$)$. Thus

$$
\left\|(-\Delta+E)^{-1}|V|\right\|_{\infty, \infty} \equiv\left\|(-\Delta+E)^{-1}|V| 1\right\|_{\infty}
$$


so (ii) is equivalent to (iii). (iv) is equivalent to (iii) because $|V|$ and $(-\Delta+E)^{-1}$ are selfadjoint and $L^{2}$ and $L^{\infty}$ are dual. (iv) $\Rightarrow$ (v) since

$$
\|V \psi\|_{1} \leqslant\left\||V|\left(H_{0}+E\right)^{-1}\right\|_{1,1}\left[\left\|H_{0} \psi\right\|_{1}+E\|\psi\|_{1}\right]
$$

and (v) implies (iv) since (A16) says that

$$
\left\|V\left(H_{0}+E\right)^{-1}\right\|_{1,1} \leqslant \varepsilon\left\|H_{0}\left(H_{0}+E\right)^{-1}\right\|_{1,1}+b\left\|\left(H_{0}+E\right)^{-1}\right\|_{1,1}
$$

and one knows that, because $H_{0}$ is the generator of a contraction semigroup on $L^{1}$

$$
\left\|H_{0}\left(H_{0}+E\right)^{-1}\right\|_{1,1} \leqslant 2 \quad\left\|\left(H_{0}+E\right)^{-1}\right\|_{1,1} \leqslant|E|^{-1} .
$$

The understanding of why one takes $\lim _{\alpha \downarrow 0}$ in the definition comes in part from the requirement that one can take $\varepsilon \rightarrow 0$ (if $b \rightarrow \infty$ ) in (A16), in part from the results on the path integral formulation of $K_{\nu}$, and in part from some continuity conditions. These conditions are responsible for the fact that $e^{-t H}$ will have only continuous functions in its range (§B3) and the result says that $K_{\nu}$ is more or less maximal for this.

PROPOSITION A.2.4. The following are equivalent:

(a) $V \in K_{\nu}^{\text {loc }}$.

(b) $f(x)=\int_{|x-y|<1}|x-y|^{-(\nu-2)}|V(y)| d^{\nu} y$ is a continuous function.

(c) If $g$ is any bounded function of compact support, then $(-\Delta+E)^{-1}[V g]$ is a continuous function.

(d) For the characteristic function $\mathcal{X}$ of any ball, $(-\Delta+E)^{-1} V \mathcal{X}_{R}$ is a compact operator from $L^{\infty}$ to $L^{\infty}$.

This result is proven in [9]. In proving $(a) \Rightarrow(b)$, a useful preliminary is to prove that if $V$ in $K_{\nu}$ has compact support, then $V$ is the limit in $K_{\nu}$-norm of bounded functions. This is done in [9] using Dini's Lemma. There is an alternative and transparent way which will be useful below. If $V$ has compact support and $\nu \geqslant 3$, let

$$
\tilde{f}(x)=\int|x-y|^{-(\nu-2)}|V(y)| d^{\nu} y
$$

i.e. if $g$ is the Coulomb potential, $|x|^{-(\nu-2)}$, then $\tilde{f}=g *(V)$. Let $h_{\delta}$ be a positive spherically symmetric $C^{\infty}$ function with $\int h_{\delta}(x) d^{\nu} x=1$ and with supp $h_{\delta} \subset\{x|| x \mid<\delta\}$. Then, by Gauss' law for Coulomb potentials, (i) $h_{\delta} * g \leqslant g$; (ii) $\left(h_{\delta} * g\right)(x)=g(x)$ if $|x|>\delta$. Thus

$$
\left\|g * h_{\delta} *|V|-g *|V|\right\|_{\infty} \leqslant \sup _{x} \int_{|x-y| \leqslant \delta}|x-y|^{-(\nu-2)}|V(y)| d^{\nu} y
$$

so $h_{\delta} *|V|$ converges to $V$ in $K_{\nu}$ norm. Since $V \in L^{1}, h_{\delta} *|V|$ is a $C^{\infty}$-function.

We are now ready to discuss some examples of functions, $V$, in $K_{\nu}$.

EXAmPLE A. Let $\mu<\nu$, let $f \in K_{\mu}$ and let $T: R^{\nu} \rightarrow R^{\mu}$ be linear and surjective. Then, we claim that $f(T x) \supseteqq V(x)$ lies in $K_{\nu}$. This is because if we integrate $|x-y|^{-(\nu-2)}$ cutoff at large $|x-y|$ over $\nu-\mu$ variables we get 
something behaving as $|T x-T y|^{-(\mu-2)}$. See [9] for a formal proof. Thus, e.g. if $f \in L^{1}, V(x) \equiv f\left(x_{1}\right)$ is in $K_{\nu}$. We summarize the moral of this example by saying that $K_{\mu} \subset K_{\nu}$.

EXAMPLE B (CENTRAL POTENTIALS). Let $V(x)=f(|x|)$ be a positive spherically symmetric function. Using the fact that such a charge distribution always produces an electrostatic potential which is maximum at $x=0$, one can see that [9]

Proposition A.2.5. If $\nu \geqslant 3$ and $V(x)=f(|x|)$, then $V \in K_{\nu}$ if and only if (i) $\sup _{y>2} \int_{|x-y|<1}|f(x)| d x<\infty$ and (ii) $\int_{0}^{2} r|f(r)| d r<\infty$.

The condition $\int_{0}^{a} r|V(r)| d r<\infty$ is very popular in analyzing the (ODE) reduced radial Schrödinger equation, see e.g. [12]. Because of the maximum at $\vec{x}=0$ fact, the finiteness of $\|V\|_{K_{\nu}}$ implies $V \in K_{\nu}$ if $V$ is spherically symmetric. In particular, if $V$ is symmetric, if $V$ is $-\Delta$-bounded in $L^{1}$ sense, then the relative bound must be 0 .

EXAMPLE C. Let $V^{*}$ be the spherical decreasing rearrangement of $V[30]$. Then, by general principles [30]

$$
\begin{aligned}
\sup _{x} \int_{|x-y|<\alpha}|x-y|^{-(\nu-2)}|V(y)| d^{\nu} y \\
\quad \leqslant \sup _{x} \int_{|x-y| \leqslant \alpha}|x-y|^{-(\nu-2)}\left|V^{*}(y)\right| d^{\nu} y .
\end{aligned}
$$

Since we have just studied when the right side goes to zero with $\alpha$ and since we can describe $V^{*}$ in terms of the distribution function $m_{V}(\lambda)=\mid\{x|| V(x) \mid \geqslant$ $\lambda\} \mid\left(|\cdot|=\right.$ Lebesgue measure), we find [9] that if $\nu \geqslant 3$ and $\int_{1}^{\infty}\left[m_{V}(\lambda)\right]^{2 / \nu} d \lambda$ $<\infty$ then $V \in K_{\nu}$.

EXAmple D (STUMmel AND Schechter SPACeS). The Schechter spaces $M_{\beta, p}$ (and their special case $M_{\beta, 2}$, the Stummel spaces) are defined in (A15). Clearly, if $\nu \geqslant 3$,

$$
M_{\beta, 1} \subset K_{\nu} \text { if } \beta>2 .
$$

Also $M_{\alpha, p} \subset M_{\beta, 1}$ if $\alpha<p \beta$ by Hölder's inequality and the fact that

$$
\int_{|x-y|<1}|x-y|^{-(1-\varepsilon) \nu} d^{\nu} x<\infty
$$

Thus

$$
M_{\alpha, p} \subset K_{\nu} \text { if } \alpha>2 p
$$

and in particular

$$
M_{\alpha, 2} \subset K_{\nu} \text { if } \alpha>4
$$

( $\alpha>4$ is precisely the condition of Stummel if $\nu \geqslant 4$ ).

EXAMPLE E ( $L^{p}$ SPACES). By (A18) and Hölder's inequality $L_{u}^{p} \subset M_{\beta, 1}$ so long as $\beta>\nu / p$ where

$$
L_{u}^{p}=\left\{\left.f\left|\sup _{x} \int_{|x-y| \leqslant 1}\right| f(x)\right|^{p} d^{\nu} x<\infty\right\} .
$$


Thus, by (A17)

$$
L_{u}^{p}\left(R^{\nu}\right) \subset K_{\nu} \text { if } p>\frac{1}{2} \nu(\nu \geqslant 2) .
$$

At the borderline $L_{u}^{\nu / 2} \not \subset K_{\nu}$; for example, a spherically symmetric function behaving as $r^{-2}(\log r)^{-\alpha}$ at $r \sim 0$ is in $L^{\nu / 2}$ if and only if $\alpha\left(\frac{1}{2} \nu\right)>1$ while $\alpha>1$ is required for it to be in $K_{\nu}$ (see Example B). As we will discuss below, for $\nu \geqslant 5 L^{\nu / 2}$ is within the regime where $-\frac{1}{2} \Delta+V$ is essentially selfadjoint but potentially not in $K_{\nu}$.

EXAMPLE F ( $N$-BODY OPERATORS). To describe $N$ particles of mass $m_{i}$ moving in $\nu$ dimensions, one studies a Hamiltonian $H$ on $R^{\nu(N-1)}$ describing motion with center of mass motion removed. It is useful to think of $X \equiv R^{\nu(N-1)}$ as points $\left(r_{1}, \ldots, r_{N}\right) \subset R^{\nu N}$ with $\Sigma m_{i} r_{i}=0 . H_{0}$ is then $\frac{1}{2}$ times the LaplaceBeltrami operator associated to the metric $m_{i}\left(d r_{i}\right)^{2}$, i.e. if $e^{(1)}, \ldots, e^{(N-1) \nu}$ are vectors in $X$ with $\left(e^{(i)}=\left(\vec{e}_{1}^{(i)}, \ldots, \vec{e}_{N}^{(i)}\right)\right)$,

$$
\sum m_{j} \vec{e}_{j}^{(i)} \cdot \vec{e}_{j}^{(k)}=\delta_{i k}
$$

and $\vec{x} \in X$ is written $\vec{x}=\Sigma x_{j} e^{(j)}$, then $H_{0}=-\frac{1}{2} \sum_{l=i}^{(n-1) \nu} \partial^{2} / \partial x_{j}^{2} . H=H_{0}+V$ with $V=\Sigma_{i<j} V_{i j}\left(r_{i}-r_{j}\right)$ and $V_{i j}$ a function on $R^{\nu}$. By Example A, $V \in K_{\nu(N-1)}$ if each $V_{i j}$ lies in $K_{\nu}$, e.g. if $V_{i j} \in L_{u}^{p}, p>\nu / 2$. For example, if $\nu=3$ and $V_{i j}(x)=e_{i j} /|x|$ (Coulomb potentials), then $V \in K_{\nu}$. Indeed $|x|^{-1}$ could be replaced by $|x|^{-\alpha}$ for any $\alpha<2$.

EXAMPle G (PERIOdic, Almost PERIOdic AND RANDOM POTENTIALS). Considerable interest has recently arisen in the study of almost periodic (see e.g. [192]) and random (see e.g. [148]) potentials. For a periodic potential to lie in $K_{\nu}$, it is obviously sufficient that it lie in $K_{\nu}^{\text {loc }}$. Almost periodic functions in the sense of Bohr are by definition continuous. For the machinery of this paper to be relevant, it is natural to extend the class and to call a function $V K_{\nu}$-almost periodic if and only if $\{V(\cdot-t)\}$ is precompact in $K_{\nu}$ norm. If one defines $L_{u}^{p}$-almost periodic in the analogous way, then for $p>\nu / 2$, any $L^{p}$-a.p. function is $K_{\nu}$-a.p. Random potentials of the type discussed in [77], i.e. continuous functions of Brownian motion on a compact manifold are bounded and so, of course, in $K_{\nu}$. But many random potentials will lead to Hamiltonians which are not bounded below and therefore the corresponding potentials will not be in $K_{\nu}$. For example, the Poisson model of [81] has this property as does various Gaussian process potentials. Typically these potentials will at least lie in $K_{\nu}^{\text {loc }}$ with probability 1.

EXAMPLE H (TRUDINGER CONDITION). In his work on Harnack inequality, Trudinger [203] (see also [72]) used the hypothesis

$$
(\varphi,|V| \varphi) \leqslant \varepsilon\left(\varphi, H_{0} \varphi\right)+C(\varepsilon)(\varphi, \varphi)
$$

for all $\varepsilon>0, \varphi \in C_{0}^{\infty}$ with

$$
C(\varepsilon) \leqslant C \varepsilon^{-M}
$$

for some $M$. It is proven in [9], that (A22) implies $V \in K_{\nu}$ (by picking $\varphi$ to be the square root of the integral kernel of $e^{-t H_{0}}$ and using Proposition A.2.6 below). It is remarkable that a purely $L^{2}$-condition on $V$ like (A22) has $L^{\infty}$ consequences (like $e^{-t H}$ maps $L^{2}$ to $L^{\infty}$ ). 
EXAMPLE I (MEASURES IN THE COMPLETION OF $K_{\nu}$ ). Let $\nu=3$. Let $\sigma$ be the measure $\mathcal{X}(x, y) \delta(z)$ with $\mathcal{X}$ the characteristic function of $\left\{x^{2}+y^{2} \leqslant 1\right\}$. Let $h_{\delta}$ be the functions discussed immediately before Example A. Let $\sigma_{\delta}=h_{\delta} * \sigma$. $\sigma_{\delta}$ is a $C^{\infty}$ function of compact support, so clearly $\sigma_{\delta} \in K_{\nu}$. We will prove that $\sigma_{\delta}$ is Cauchy, converging in a real sense to $\sigma$. Among other things this implies that $K_{\nu}$ is not complete. We begin, by noting that by a simple argument

$$
f_{\alpha}(x) \equiv \int_{|x-y| \leqslant \alpha}|x-y|^{-1} d \sigma(y)
$$

takes its maximum at $x=0$ and

$$
f_{\alpha}(0)=2 \pi \int_{0}^{\alpha} d r=2 \pi \alpha .
$$

But as in the argument before Example A, by Gauss's law, if $\delta<\delta^{\prime}$, then

$$
\left\|\sigma_{\delta}-\sigma_{\delta^{\prime}}\right\|_{K_{3}} \leqslant \sup _{x} f_{\delta^{\prime}}(x)=2 \pi \delta^{\prime}
$$

proving the desired result.

Probably, one could develop a more systematic theory by allowing $V$ to be a measure obeying the proper condition, but we do not see the purpose of the extra complication. In some sense, the point is that $K_{\nu}^{\text {loc }}$ is the set of functions which are distributional Laplacians of continuous functions. Of course, not every such distribution is a function, in fact, not every such distribution is a measure (e.g. if $\nu=1$, the principal part is such a distribution).

Next, we turn to the critical connection of $K_{\nu}$ with functional integrals. $E_{x}$ will denote expectation for the Brownian motion starting at $x \in R^{\nu}$, i.e. $b(t)$ is an $R^{\nu}$ valued Gaussian process with

$$
E_{x}\left(b_{j}(t)\right)=x_{j}, \quad E_{x}\left(\left[b_{j}(t)-x_{j}\right]\left[b_{k}(s)-x_{k}\right]\right)=\delta_{j k} \min (s, t) .
$$

See [194] for discussion of the construction of this process. The real point of the definition is that for $0<s_{1}<s_{2}<\cdots<s_{n}$,

$$
E_{x}\left(f_{1}\left(b\left(s_{1}\right)\right) \cdots f_{n}\left(b\left(s_{n}\right)\right)\right)=\left\{e^{-t_{1} H_{0}}\left[f_{1} \cdots e^{-t_{n} H_{0}} f_{n}\right]\right\}(x)
$$

where $t_{i}=s_{i}-s_{i-1}\left(s_{0}=0\right)$ and the first $f_{n}$ is viewed as a function to which $e^{-t_{n} H_{0}}$ is applied and the remaining $f_{i}$ 's are treated as multiplication operators. In (A24), $H_{0}=-\frac{1}{2} \Delta$ and the reason for the $\frac{1}{2}$ in our definition is that (A24) should hold with the conventional normalization (A23) of $E_{x}$.

$K_{\nu}$ has a natural expression in terms of $E_{x}$ :

Proposition A.2.6 [9]. $V \in K_{\nu}$ if and only if

$$
\lim _{t \downarrow 0} \sup _{x} E_{x}\left(\int_{0}^{t}|V(b(s))| d s\right)=0 \text {. }
$$

The proof [9] depends on the fact that

$$
E_{x}\left(\int_{0}^{t}|V(b(s))| d s\right)=\int Q_{t}(x-y)|V(y)| d^{\nu} y
$$


with $Q_{t}(x)=\int_{0}^{t}(2 \pi s)^{-\nu / 2} \exp \left(-x^{2} / 2 s\right)$. Moreover $Q_{t}(x)$ for fixed $t$ looks like $c|x|^{-(\nu-2)}$ for $|x|$ small and $Q_{t}(x)$ is very small outside the region $|x| \gg \sqrt{t}$ so $\int Q_{t}(x-y)|V(y)| d^{\nu} y$ behaves as $t \rightarrow 0$ like

$$
\int_{|x-y|<\sqrt{t}}|x-y|^{-(\nu-2)}|V(y)| d^{\nu} y .
$$

One nice feature of (A25) is that it makes the assertion in Example A particularly transparent, since if $V$ is a function on $R^{\nu}$ depending only on an $R^{\mu}$ in $R^{\nu}$, the $R^{\mu}$-restriction of $\nu$-dimensional Brownian motion is precisely $\mu$-dimensional Brownian motion.

Brownian motion is important in the study of the semigroup $e^{-t H}$ because of the Feynman-Kac formula

$$
\left(e^{-t H} f\right)(x)=E_{x}\left(\exp \left(-\int_{0}^{t} V(b(s)) d s\right) f(b(t))\right) .
$$

The question of justifying this formula leads us to the final question of this section, namely the definition of $H$ and the proof of (A26). There are basically three approaches:

(1) [The approach used by McKean [133].] Prove the integrability of the quantity $\exp \left(-\int_{0}^{t} V(b(s)) d s\right) f(b(s))$ for $f \in C_{0}^{\infty}$ and that the right side of (A26) defines a bounded operator $A_{t}$ on $L^{2}$ (we essentially do this in §B1), and then show that $A_{t}$ is a strongly continuous semigroup by exploiting the Markov property of Brownian motion (see [133] for details). Thus $A_{t}=e^{-t H}$ for some selfadjoint $H$. That is one uses (A26) to define $H$.

(2) If $V_{-} \in K_{\nu}$, then by Proposition A.2.3, $\left\|\left|V_{-}\right|\left(H_{0}+E\right)^{-1}\right\|_{1,1} \rightarrow 0$ as $E \rightarrow \infty$. Taking adjoints $\left\|\left(H_{0}-E\right)^{-1}\left|V_{-}\right|\right\|_{\infty, \infty} \rightarrow 0$ as $E \rightarrow \infty$. Thus, by the Stein interpolation theorem

$$
\left\|\left|V_{-}\right|^{1 / 2}\left(H_{0}+E\right)^{-1 / 2}\right\|_{2,2}^{2} \equiv\left\|\left|V_{-}\right|^{1 / 2}\left(H_{0}+E\right)^{-1}\left|V_{-}\right|^{1 / 2}\right\|_{2,2} \rightarrow 0
$$

as $E \rightarrow \infty$, i.e. $V_{-}$is the $H_{0}$-form bounded with relative bound zero, so if $V_{+} \in K_{\nu}^{\text {loc }}, H_{0}+V$ defines a closed form on $Q\left(H_{0}\right) \cap Q\left(V_{+}\right)$. (A26) can then be proven, initially for nice $V$ and then by a limiting argument; see [194] for details.

(3) Use the final steps of (2), but prove that $V_{-}$is $H_{0}$-form bounded by using the arguments in $\S \mathrm{B} 1$. To be more explicit: if $V$ is bounded and continuous, one proves (A26) by various methods (see [194]); use the arguments of $\S \mathrm{B} 1$ to get a bound on $\left\|\exp \left[-t\left(H_{0}+\alpha V\right)\right]\right\|_{2,2}$ depending only on $\left\|V_{-}\right\|_{K_{v}}$ and so a lower bound on $H_{0}+\alpha V$ depending only on this norm. Then by a limiting argument using the fact that the bounded continuous functions are dense in $K_{\nu}$, one gets (A26) and simultaneously the form bound for all $V_{-}$ in $K_{v}$.

The point, of course, is that all the above lead to the same $H$ since all obey (A26). We summarize with

TheOREM A.2.7. Let $V_{-} \in K_{v}, V_{+} \in K_{v}^{\text {loc }}$. Then the quadratic form $H_{0}+V$ is closed on $Q\left(V_{+}\right) \cap Q(-\Delta)$ and defines a semibounded operator $H$. The semigroup $e^{-t H}$ obeys (A26). 
In $\S \mathrm{B} 1$ we sketch the proof of the following facts about form and operator cores for $H$.

TheOREM A.2.8 (三 THEOREM B.1.5) (Simon [187]). Under the hypothesis of Theorem A.2.7, $C_{0}^{\infty}$ is a form core for $H$, i.e. given $\varphi \in Q(H)$, we can find $\varphi_{n} \in C_{0}^{\infty}$ so that $\left\|\varphi-\varphi_{n}\right\|_{2} \rightarrow 0,\left\langle\left(\varphi-\varphi_{n}\right), H\left(\varphi-\varphi_{n}\right)\right\rangle \rightarrow 0$.

TheOrem A.2.9 (= TheOREM B.1.6) (KATo [112]). Under the hypotheses of Theorem A.2.9, if $V$ is also in $L_{\text {loc }}^{2}$, then $C_{0}^{\infty}$ is an operator core for $H$, i.e. given $\varphi \in D(H)$, we can find $\varphi_{n} \in C_{0}^{\infty}$ so that $\left\|\varphi_{n}-\varphi\right\|_{2} \rightarrow 0$ and $\left\|H \varphi-H \varphi_{n}\right\|_{2} \rightarrow$ 0 .

REMARKS. 1. The condition $V_{-} \in K_{\nu}$ is explicitly given in Kato's paper [112] ( $V_{+} \in K_{\nu}^{\text {loc }}$ is not given, only $V_{+} \in L_{\text {loc }}^{2}$ and indeed, it is not needed). Simon [187] does not give the condition explicitly and indeed requires $V_{-}=0$ but his method works for $V_{-} \in K_{\nu}$ as he noted in [190], see $\S B .1$.

2. See $\S B .13$ for the analogous theorem with magnetic field.

A3. Literature on larger classes. As we have already indicated, the class of potentials, even negative ones, for which $H$ has a reasonable definition, is larger than the class $K_{\nu}$; e.g. for $V(x) \sim-c|x|^{-2}[-\log |x|]^{-\alpha}$ for $|x|$ small, we have (if $\nu \geqslant 3$ ), that $V$ is in $K_{\nu}$ only if $\alpha>1$ but that $V$ is form bounded with relative bound zero if $\alpha>0$. $K_{\nu}$ is exactly the border for bounded eigenfunctions; indeed $V$ 's with $\alpha=1$ can have eigenfunctions diverging as $\left[\log |x|^{-1}\right]^{\beta}$ as $|x| \sim 0$. Of course for potentials not in $K_{\nu}$ where eigenfunctions may not be in $L^{\infty}$, one can ask about $L^{p}$-properties. Because the class of potentials involved is so "thin" and includes none of physical interest, I regard results of this genre as having rather limited significance but in a comprehensive review, we should give appropriate references.

For definition of $H$ in wider classes, see e.g. [46, 108, 109, 161].

For potentials where $V$ is $L^{1}-\Delta$-bounded with nonzero relative bound (there are no such potentials among the spherically symmetric ones, but one can construct pathological examples) and where eigenfunctions can be bounded, see $[123,150]$.

As for $L^{p}$-properties of eigenfunctions, there is a general result of Brezis-Kato [31].

THEOREM A.3.1 [31]. If $V_{-}$is $\left(L^{2}\right)-\Delta$-bounded with relative bound zero, then any eigenfunction is locally in $\bigcap_{p<\infty} L^{p}\left(R^{\nu}\right)$.

See [122] for discussion of eigenfunctions when $V \in L_{w}^{\nu / 2}\left(R^{\nu}\right)$.

\section{B. $L^{p}$-PROPERTIES}

B1. $L^{p}$-smoothing of semigroups. In this section we prove the following basic results:

THEOREM B.1.1 $[34,123,194]$. Let $V_{-} \in K_{\nu}, V_{+} \in K_{\nu}^{\text {loc }}$. Then for every $t>0$ and $p \leqslant q, e^{-t H}$ is bounded from $L^{p}$ to $L^{q}$.

REMARKS. 1. Since $e^{-t H}|f|$ is pointwise monotone decreasing as $V_{+}$increases, $V_{+}$can be more singular without affecting the result. 
2. As far as local singularities are concerned this is an expression that $e^{-t H}$ is "smoothing".

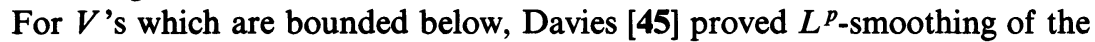
semigroup in 1973. The first general results on $L^{p}$-smoothing were obtained by Herbst and Sloan [83] but with different hypothesis on $V$ and often weaker conclusions. They also noted the importance to the existence of integral kernels and to the existence of BGK eigenfunction expansions. Their germinal paper motivated later work. The method of Kovelenko and Semenov [34] exploits semigroup analytical methods and will be briefly discussed later. Carmona [123] and Simon [194] independently found the proof we give here. They relied on a lemma which goes back at least to a paper of Khasmin'skii [115] and which was later rediscovered by Portenko [156] and by Berthier and Gaveau [27]. The last paper was the direct motivation of $[123,194]$.

LEMMA B.1.2 (KhASMIN'SKII's LEMMA [115]). Let $f \geqslant 0$ be a function on $R^{\nu}$ with

$$
\alpha \equiv \sup _{x} E_{x}\left(\int_{0}^{t} f(b(s)) d s\right)<1
$$

for some $t$. Then

$$
\sup _{x} E_{x}\left(\exp \left(\int_{0}^{t} f(b(s)) d s\right)\right) \leqslant(1-\alpha)^{-1} .
$$

PROof [115]. By expanding the exponential, it suffices to prove that

$$
\sup _{x} E_{x}\left(\int_{0<s_{1}<\cdots<s_{n}<t} d s_{1} \cdots d s_{n} f\left(b\left(s_{1}\right)\right) \cdots f\left(b\left(s_{n}\right)\right)\right) \leqslant \alpha^{n} .
$$

Fixing $s_{1}, \ldots, s_{n-1}$, it obviously suffices, by induction, to show that

$$
E_{x}\left(\int_{s_{n-1}}^{t} d s_{n} f\left(b\left(s_{1}\right)\right) \cdots f\left(b\left(s_{n}\right)\right)\right) \leqslant \alpha E_{x}\left(f\left(b\left(s_{1}\right)\right) \cdots f\left(b\left(s_{n-1}\right)\right)\right) .
$$

By the Markov property, and the starting afresh of Brownian motion, we can condition on the path up to time $s_{n-1}$ and find that the left side of (B4) equals

$$
E_{x}\left(f\left(b\left(s_{1}\right)\right) \cdots f\left(b\left(s_{n-1}\right)\right) E_{b\left(s_{n-1}\right)}\left(\int_{0}^{t-s_{n-1}} f(b(u)) d u\right)\right) .
$$

Since $f$ is positive, using (B1), we can bound the last function by $\alpha$ and so obtain (B4).

RemarKs. 1. There is an analytic version of this argument, due to Kato [111], who introduced it in a different context.

2. (B1) and Proposition A.2.6 show why $K_{\nu}$ enters naturally.

The above proof does not require the equality of the functions $f$ in (B3) and we note the following immediate extension which will be very useful later (§B10).

LEMMA B.1.3. Let

$$
\alpha_{i}=\sup _{x} E_{x}\left(\int_{0}^{t}\left|f_{i}(b(s))\right| d s\right) ; \quad i=1, \ldots, n .
$$


Then

(B5) $\sup _{x} E_{x}\left(\int_{0<s_{1}<\cdots<s_{n}<t}\left|f_{1}\left(b\left(s_{1}\right)\right) \cdots f_{n}\left(b\left(s_{n}\right)\right)\right| d s_{1} \cdots d s_{n}\right) \leqslant \prod_{1}^{n} \alpha_{i}$.

Proof of Theorem B.1.1 [123, 194]. As a preliminary, we note that by duality and interpolation, it suffices to show that $e^{-t H}$ is bounded from $L^{\infty}$ to $L^{\infty}$ and $L^{1}$ to $L^{\infty}$.

Step $1\left(L^{\infty}\right.$ to $\left.L^{\infty}\right)$. By the Feynman-Kac formula

$$
\begin{aligned}
\left\|e^{-t H} f\right\|_{\infty} & =\sup _{x} E_{x}\left(\exp \left(-\int_{0}^{t} V(b(s)) d s\right) f(b(t))\right) \\
& \leqslant\|f\|_{\infty} \sup _{x} E_{x}\left(\exp \left(\int_{0}^{t}\left|V_{-}(b(s))\right| d s\right)\right) \leqslant C\|f\|_{\infty}
\end{aligned}
$$

if $t$ is sufficiently small by using Proposition A.2.6, the hypothesis $V_{-} \in K_{\nu}$ and Lemma B.1.2. Thus for some $T$,

$$
\sup _{0<t<T}\left\|e^{-t H}\right\|_{\infty, \infty} \leqslant C .
$$

Letting $A=T^{-1} \ln C$, we obtain by using the semigroup property

$$
\left\|e^{-t H}\right\|_{\infty, \infty} \leqslant C \exp (A t)
$$

By the same argument, we see that

$$
\left\|e^{-t\left(H_{0}+2 V\right)}\right\|_{\infty, \infty} \leqslant \tilde{C} \exp (\tilde{A} t)
$$

Step $2\left(L^{2}\right.$ to $\left.L^{\infty}\right)$. By using the Schwarz inequality in the Feynman-Kac formula, we obtain

$$
\left|\left(e^{-t H} f\right)(x)\right| \leqslant E_{x}\left(\exp \left(-2 \int_{0}^{t} V(b(s)) d s\right)\right)^{1 / 2} E_{x}\left(|f(b(t))|^{2}\right)^{1 / 2}
$$

so using the Feynman-Kac formula again, we find that

$$
\left|\left(e^{-t H} f\right)(x)\right| \leqslant\left[\left(e^{-t\left(H_{0}+2 V\right)} 1\right)(x)\right]^{1 / 2}\left[e^{-t H_{0}}|f|^{2}\right]^{1 / 2} \text {. }
$$

But $e^{-t H_{0}}$ is convolution by an explicit function $(2 \pi t)^{-\nu / 2} \exp \left(-|x|^{2} / 2 t\right)$. Since this function is in $L^{\infty}$, we have that

$$
\left\|e^{-t H_{0}} g\right\|_{\infty} \leqslant(2 \pi t)^{-\nu / 2}\|g\|_{1}
$$

so by (B7)

$$
\left\|e^{-t H} f\right\|_{\infty} \leqslant \tilde{C}^{1 / 2} \exp \left(\frac{1}{2} \tilde{A} t\right)(2 \pi t)^{-\nu / 4}\|f\|_{2} .
$$

Step 3 ( $L^{1}$ to $L^{2}$ ). By selfadjointness of $e^{-t H}$, we have that

$$
\left\|e^{-t H}\right\|_{1,2}=\left\|e^{-t H}\right\|_{2, \infty} .
$$

Step $4\left(L^{1}\right.$ to $\left.L^{\infty}\right)$. By the semigroup property,

$$
\left\|e^{-t H}\right\|_{1, \infty} \leqslant\left\|e^{-t H / 2}\right\|_{1,2}\left\|e^{-t H / 2}\right\|_{2, \infty}
$$

so by (B9)

$$
\left\|e^{-t H}\right\|_{1, \infty} \leqslant \tilde{C} \exp \left(\frac{1}{2} \tilde{A} t\right)(\pi t)^{-\nu / 2}
$$


Making explicit the result of exploiting interpolation, from (B6) and (B10), we find that for $p \leqslant q$

$$
\left\|e^{-t H}\right\|_{p, q} \leqslant C^{\prime} t^{-\gamma} \exp \left(A^{\prime} t\right)
$$

where

$$
\gamma=\frac{1}{2} \nu\left\{p^{-1}-q^{-1}\right\}
$$

Later (\$B5), we will show that one can take $A^{\prime}$ to be any number larger than -inf $\operatorname{spec}_{L^{2}}(H)$ where $\operatorname{spec}_{L^{2}}$ is the $L^{2}$-spectrum of $H$. We will use this fact when we exploit (B11) in the next section.

The above clearly proves half of Theorem A.2.1. There is the other half:

Proposition B.1.4. If $V \leqslant 0$ and $\lim _{t \downarrow 0}\left\|e^{-t H}\right\|_{\infty, \infty}=1$, then $V \in K_{\nu}$.

Proof. By Jensen's inequality, since $V \leqslant 0$,

$$
\begin{aligned}
1 & \leqslant \sup _{x} \exp \left\{E_{x}\left(\int_{0}^{t}|V(b(s))| d s\right)\right\} \leqslant \sup _{x} E_{x}\left(\exp \left(-\int_{0}^{t} V(b(s)) d s\right)\right) \\
& =\left\|e^{-t H} 1\right\|_{\infty}=\left\|e^{-t H}\right\|_{\infty, \infty}
\end{aligned}
$$

so, by the hypothesis

$$
\lim _{t \downarrow 0} \sup _{x} E_{x}\left(\int_{0}^{t}|V(b(s))| d s\right)=0 .
$$

By Proposition A.2.6, $V \in K_{\nu}$.

Theorem B.1.1 has at least two distinct analytic proofs:

(1) There is an analytic translation of the probabilistic proof (see e.g. [50]). Khasmin'skii's lemma as already noted has a "Kato-smoothness" analytic version. The key inequality (B8) can be proven without path integrals by a "complex interpolation argument" (see e.g. [50]).

(2) There is a semigroup proof [34]. $V$ in $K_{\nu}$ implies that $V$ is an $L^{2}$-relatively bounded perturbation of $H_{0}$ with relative bound 0 (see Proposition A.2.3). Since $e^{-t H_{0}}$ is an analytic semigroup on $L^{1}$ (using the explicit integral kernel $e^{-z H_{0}}$ is defined as a map from $L^{1}$ to $L^{1}$ so long as $\operatorname{Re} z>0$ and for each $\left.\theta<\pi / 2, \sup _{|\arg z|<\theta}\left\|e^{-z H_{0}}\right\|_{1,1}<\infty\right)$, the same is true of $e^{-t H}$. It follows that $\left\|e^{-t H}\right\|_{1,1} \leqslant C \exp (A t)$ and $\operatorname{Ran}_{L^{1}}\left(e^{-t H}\right) \subset D_{L^{1}}(H)=D_{L^{1}}\left(H_{0}\right)$; the latter equality is a consequence of the relative bound result and the inclusion is a general property of analytic semigroups. By a Sobolev estimate, $D_{L^{1}}\left(H_{0}\right) \subset L^{p}$ if $p<\nu /(\nu-2)(p<\infty$ if $\nu=1,2)$. Thus, $e^{-t H}$ maps $L^{1}$ to $L^{p_{0}}$ for some $p_{0}>1$. Without loss, we suppose that $p_{0}^{-1}=1-1 / n$ for an integer $n$. By duality and interpolation $e^{-t H}$ maps $L^{p}$ to $L^{q}$ if $p^{-1}-q^{-1}=n^{-1}$ so $e^{-n t H}$ maps $L^{1}$ to $L^{\infty}$. This completes our sketch of the proof of [34].

As a final result in this section, we sketch the proofs of Theorems A.2.8 and A.2.9 following [187].

THEOREM B.1.5. Let $V_{-} \in K_{\nu}, V_{+} \in K_{\nu}^{\text {loc }}$ and let $H$ be the semigroup defined by the quadratic form $H_{0}+V$ on $Q(V) \cap Q\left(H_{0}\right)$. Then $C_{0}^{\infty}$ is a form core for $H$. 
REMARK. As the proof will show, only $V_{+}$in $L_{\text {loc }}^{1}$ is needed.

SKETCH (FOLLOWING [187]). $e^{-H}\left[L^{2}\right]$ is certainly a form core for $H$, but by Theorem B.1.1 this is in $L^{\infty}$, i.e. (since $Q\left(H_{0}\right) \supset Q(H)$ ) we see that $\mathscr{D} \equiv L^{\infty} \cap$ $L^{2} \cap Q\left(H_{0}\right) \cap Q\left(V_{+}\right)$is a form core for $H$. Thus, given $\varphi$ in this set we need only approximate $\varphi$ by functions in $C_{0}^{\infty}$ in form norm. Pick any $j \in C_{0}^{\infty}$ with $j(0)=1$ and let $j_{n}(x)=j(x / n)$ and $\varphi_{n}=j_{n} \varphi$. By dominated convergence $\varphi_{n} \rightarrow \varphi$ in $L^{2}$ and $V_{+}^{1 / 2} \varphi_{n} \rightarrow V_{+}^{1 / 2} \varphi$ in $L^{2}$. Moreover, $\nabla \varphi_{n}=j_{n}(\nabla \varphi)+$ $\varphi n^{-1}(\nabla j)(x / n)$ converges to $\nabla \varphi$ by dominated convergence again. Thus we need only prove that the functions in $\mathscr{D}$ which have compact support can be approximated by functions in $C_{0}^{\infty}$. Given $\psi$ in this set, pick $k \in C^{\infty}$ with $k \geqslant 0$ and $\int k(x) d x=1$. Let $k^{(n)}(x)=n k(x n)$ and $\psi^{(n)}=k^{(n)} * \psi$. Clearly $\psi^{(n)} \in$ $C_{0}^{\infty}$ and since $\psi \in L^{\infty}$ (this is where Theorem B.1.1 enters critically) and $V \in L_{\mathrm{loc}}^{1}, \psi^{(n)} \rightarrow \psi$ in form norm.

TheOREM B.1.6 (TheOREM A.2.9). Let $V_{-} \in K_{\nu}, V_{+} \in K_{\nu}^{\text {loc }}$ and $V$ in $L_{\text {loc }}^{2}$ and let $H$ be as defined in the previous theorem. Then $C_{0}^{\infty}$ is an operator core for $\boldsymbol{H}$.

REMARK. As the proof shows only $V_{+}$in $L_{\text {loc }}^{2}$ is required.

Proof. The proof is identical to that of the last theorem with one preliminary step. Namely, to prove that $\varphi_{n} \rightarrow \varphi$ in operator graph norm, one needs

$$
H\left(j_{n} \varphi\right)=j_{n} H \varphi+\nabla j_{n} \cdot \nabla \varphi-\frac{1}{2}\left(\nabla j_{n}\right) \varphi
$$

and this is proven by showing that both sides have the same inner product with a function in $C_{0}^{\infty}$ and the already proven density of $C_{0}^{\infty}$ in form norm.

REMARKS. 1. We emphasize that the conditions $V$ in $L_{\text {loc }}^{1}$ (resp. $V$ in $L_{\text {loc }}^{2}$ ) are necessary for $C_{0}^{\infty}$ to lie in $Q(H)$ (resp. $D(H)$ ). Thus, as far as $V_{+}$is concerned the above results with $K_{\nu}^{\text {loc }}$ dropped are "maximal".

2. As mentioned already, $K_{\nu}$ is not the maximal condition for $V_{-}$as far as form cores and operator cores are concerned. However, since the above proof uses the $L^{2}$ to $L^{\infty}$ result, $V_{-} \in K_{\nu}$ is the maximal class for the above proof. To the extent that Kato's inequality has a "semigroup nature" (see [184, 84, 185]), one understands why Kato was led to introduce $K_{\nu}$.

B2. Sobolev estimates. Sobolev estimates for Schrödinger operators, by which we mean $L^{p}$-bounds for the resolvent of $H$, are an immediate consequence of (B11).

TheOREM B.2.1. Let $H=H_{0}+V$ with $V_{+} \in K_{\nu}^{\text {loc }}, V_{-} \in K_{\nu}$. Let $\alpha>0$ and let $p, q$ obey $p \leqslant q$ and

$$
p^{-1}-q^{-1}<(2 \alpha / \nu)
$$

Let $z$ be a complex number obeying $\operatorname{Re} z<\inf \operatorname{spec}(H)$. Then $(H-z)^{-\alpha}$ is bounded from $L^{p}$ to $L^{q}$.

REMARKS. 1. If $\alpha$ is an integer, $(H-z)^{-\alpha}$ means a power of the conventional resolvent. For nonintegral $\alpha$, one can define $(H-z)^{-\alpha}$ either by (A9) or, equivalently, by defining it on $L^{2} \cap L^{p}$ for $z$ real by the usual $L^{2}$ functional calculus and analytic continuation.

2. As emphasized already inf $\operatorname{spec}(H)$ (more properly inf $\operatorname{Re}[\operatorname{spec}(H)]$ since we do not know that $\operatorname{spec}(H)$ is real for $p \neq 2$ ) is independent of $p$; see $\S \mathrm{B} 5$. 
Proof. For the $\|\cdot\|_{p, q}$ of the integrand of (A9) to converge, one uses (B11) and finds that $\alpha>\gamma$ is needed. This is precisely (B12).

Open questions. Does the result remain true if $p^{-1}-q^{-1}=(2 \alpha / \nu) ; 1<p<$ $q<\infty$ ? Since it is true if $V=0$, one would expect that the answer is yes. If it is yes, under what circumstances can one take $z$ to inf $\operatorname{spec}(H)$ and remain bounded from $L^{p}$ to $L^{q}$ (as one can when $V=0$ )? Since inf $\operatorname{spec}(H)$ may be an eigenvalue, one cannot always do that.

As for $z$ not obeying $\operatorname{Re} z<\inf \operatorname{spec}(H)$, the resolvent equation (with $w=\inf \operatorname{spec}(H)-1)$

$$
(H-z)^{-1}=(H-w)^{-1}+(z-w)(H-w)^{-1}(H-z)^{-1}
$$

immediately implies (a) and (b) below; (c) is a corollary of Theorem B.2.3 below.

THEOREM B.2.2. (a) Suppose that $H, p, q$ obey the hypotheses of Theorem B.1.1 with $\alpha=1$. Suppose that $z \notin \operatorname{spec}_{L^{p}}(H)$. Then $(H-z)^{-1}$ is bounded from $L^{p}$ to $L^{q}$.

(b) Suppose that $H, p, q$ obey the hypotheses of Theorem B.1.1 with $\alpha$ an integer, $m$. Suppose $z \notin \cup_{r} \operatorname{spec}_{L^{r}}(H)$. Then $(H-z)^{-m}$ is bounded from $L^{p}$ to $L^{q}$.

(c) (b) is true only if $z \notin \operatorname{spec}_{L^{2}}(H)$, so long as $p \leqslant 2 \leqslant q$.

REMARK. We do not know that $\operatorname{spec}_{L^{r}}(H)$ is $r$ independent.

Open question. What can one say about $\alpha$ nonintegral (where $(H-z)^{-\alpha}$ will have to be defined by some kind of analytic continuation)?

Thus far we have discussed $f(H)$ for $f(x)$ equals $e^{-t H}$ and $(x-z)^{-\alpha}$. One result for general $f$ is easy.

TheOREM B.2.3. Suppose $1 \leqslant p \leqslant 2 \leqslant q \leqslant \infty$ obey (B12) for some $\alpha$. Let $f$ be a Borel function on $L^{2}-\operatorname{spec}(H)$ obeying

$$
|f(x)| \leqslant C(|x|+1)^{-\alpha} ; \quad x \in \operatorname{spec}(H) .
$$

Then $f(H)$ is bounded from $L^{p}$ to $L^{q}$.

REMARK. Since $f(H)$ is only a priori defined on $L^{2}$, one should really say that for any $\psi \in L^{p} \cap L^{2}$, the $L^{2}$-function $f(H) \psi$ lies in $L^{q}$ and $\|f(H) \psi\|_{q} \leqslant$ $c\|\psi\|_{p}$ whence $f(H)$ can be defined from $L^{p}$ to $L^{q}$.

Proof. Let $g(x)=(x-w)^{\alpha} f(x)$ with $w=\inf \operatorname{spec}(H)-1$. Pick $\alpha^{\prime}, \alpha^{\prime \prime}$ so $\alpha=\alpha^{\prime}+\alpha^{\prime \prime}$ and $p^{-1}-\frac{1}{2}<2 \alpha^{\prime} / \nu$ and $\frac{1}{2}-q^{-1}<2 \alpha^{\prime \prime} / \nu$. Write $f(H)=$ $(H-w)^{-\alpha^{\prime \prime}} g(H)(H-w)^{-\alpha^{\prime}}$ and use the facts that $g(H)$ is bounded on $L^{2}$ and $(H-w)^{-\alpha^{\prime}}$ (resp. $\left.(H-w)^{-\alpha^{\prime \prime}}\right)$ is bounded from $L^{p}$ to $L^{2}$ (resp. $L^{2}$ to $L^{q}$ ).

The requirement $p \leqslant 2 \leqslant q$ is disappointing, but at least the case $p=1$, $q=\infty$ needed to get bounded integral kernels is included.

Open question. What can one say if $p<q<2$ or $2<p<q$ ?

B3. Continuity and derivative estimates. Thus far we have discussed the $L^{p}$-properties of $\operatorname{Ran}\left(e^{-t H}\right)$. In this section, we want to show that $\operatorname{Ran}\left(e^{-t H}\right)$ lies in the continuous functions and in those functions whose distributional derivative is locally in $L^{2}$. These results are new. 
TheOREM B.3.1. Let $V_{-} \in K_{\nu} ; V_{+} \in K_{\nu}^{\text {loc }}$. Let $f \in L^{\infty}$. Then $e^{-t H} f$ is $a$ continuous function.

PROOF. In $\S \mathrm{B} 10$, we will prove that for any $V$ with these properties, one can find $V_{n} \in C_{0}^{\infty}$ so that for any compact set $K$,

$$
\lim _{n \rightarrow \infty}\left\|\mathscr{X}_{K}\left[e^{-t H}-e^{-t H_{n}}\right]\right\|_{\infty, \infty}=0
$$

where $H_{n}=H_{0}+V_{n}$ and $\mathscr{X}_{K}$ is the characteristic function of $K$. Thus $e^{-t H_{n}} f$ converges to $e^{-t H} f$ uniformly on compacts, so we can suppose $V \in C_{0}^{\infty}$ without loss of generality.

The result in this case can be proven in many ways. Here is one: Since $e^{-t H}$ maps $L^{\infty}$ to $L^{\infty}$, we can suppose without loss that $t$ is so small that

$$
\sup _{x} E_{x}\left(\int_{0}^{t}|V(b(s))| d s\right)=\alpha<1 .
$$

By the argument in $§ B .1$, it suffices to prove that

$$
E_{x}\left(\int_{0<s_{1}<\cdots<s_{n}<t} V\left(b\left(s_{1}\right)\right) \cdots V\left(b\left(s_{n}\right)\right) f(b(t))\right) d s_{1} \cdots d s_{n}
$$

is continuous in $x$. Let $x_{n} \rightarrow x$ and let

$$
G(y, b)=V\left(y+b\left(s_{1}\right)\right) \cdots V\left(y+b\left(s_{n}\right)\right), \quad F(y, b)=f(y+b(t)) .
$$

We want to prove that $E_{0}\left(G\left(x_{n}, b\right) F\left(x_{n}, b\right)\right) \rightarrow E_{0}(G(x, b) F(x, b))$. Since $F$ and $G$ are uniformly bounded, it suffices that

$$
\begin{aligned}
& E_{0}\left(\left|G\left(x_{n}, b\right)-G(x, b)\right|\right) \rightarrow 0, \\
& E_{0}\left(\left|F\left(x_{n}, b\right)-F(x, b)\right|\right) \rightarrow 0 .
\end{aligned}
$$

(B13) follows from dominated convergence. (B14) says that

$$
\int(2 \pi t)^{-\nu / 2} \exp \left(-y^{2} / 2 t\right)\left|f(x+y)-f\left(x_{n}+y\right)\right| d y
$$

goes to zero if $f \in L^{\infty}$. This is easy to prove, for example, by writing $f$ as the sum of a continuous function, a function in $L^{1}$ with very small $L^{1}$-norm and a function in $L^{\infty}$ supported very far from $-x$.

By the semigroup property and Theorem B.1.1, we have

Corollary B.3.2. If $V_{+} \in K_{\nu}^{\text {loc }}, V_{-} \in K_{\nu}$ and $f \in L^{p}$ for any $p \in[1, \infty]$, then $e^{-t H} f$ is a continuous function.

Since $\int_{t}^{\infty} g(s) e^{-s H} d s=e^{-t H} \int_{0}^{\infty} g(s+t) e^{-s H} d s$ and $\left\|\int_{0}^{t} g(s) e^{-s H} d s\right\| \rightarrow 0$ as $t \downarrow 0$ if $\int_{0}^{\infty}|g(s)|\left\|e^{-s H}\right\| d s<\infty$, we immediately have

TheOREM B.3.3. Let $V_{+} \in K_{\nu}^{\text {loc }}, V_{-} \in K_{\nu}$ and let $p>\nu / 2 \alpha$. Then for any $z$ with $\operatorname{Re} z<\inf \operatorname{spec}(H)$, we have that $(H-z)^{-\alpha}$ maps $L^{p}$ to the bounded continuous functions.

Next, we want to turn to properties of $\nabla \varphi$. It is useful to begin with an example. 
EXAMPLE $(\nu=2)$. Let $f(x)$ be a function on $[0, \infty]$ which is positive and $C^{\infty}$ on $(0, \infty)$, equal to $r^{-1 / 2} e^{-r}$ for $r>1$ and which equals $1-r^{\gamma}$ for $r<\frac{1}{2}$. Let $\psi(x)=f(|x|)$ and $V(x)=\frac{1}{2}+\frac{1}{2} \Delta \psi / \psi$, so $V$ is $C^{\infty}$ away from 0 , has compact support and $V(x)=\frac{1}{2}-\frac{1}{2} \gamma^{2}\left(1-r^{\gamma}\right)^{-1} r^{\gamma-2}$ for $|x|=r$ small. Thus $V \in K_{\nu}$ for any $\gamma>0$. But, when $\gamma<1, \nabla \psi \in L^{p}$ only if $p<2 /(1-\gamma)$. As $\gamma \downarrow 0$, the critical $p$ goes to 2 so we can only hope for $L_{\text {loc }}^{2}$ results for $\nabla \psi$ when $\nu=2$. Since $K_{2} \subset K_{\nu}$ if $\nu>2$, the same is true for any $\nu \geqslant 2$. For $\nu=1$, easy methods show that $e^{-t H} f$ is $C^{1}$ in the classical sense.

That $\nabla \varphi \in L_{\text {loc }}^{2}$ if $\varphi=e^{-t H} f$ is certainly to be expected. After all if $f \in L^{2}$, then $\varphi \in Q(H) \subset Q\left(H_{0}\right)=\left\{\varphi \in L^{2} \mid \nabla \varphi \in L^{2}\right\}$.

Proposition B.3.2. Let $V \in K_{\nu}^{\mathrm{loc}}$. Let $f \in L_{\mathrm{loc}}^{\infty}$ so that $-\Delta f$ and $V f$ can be defined as distributions. Suppose $-\frac{1}{2} \Delta f+V f$ is in $L_{\mathrm{loc}}^{\infty}$. Then $\nabla f \in L_{\mathrm{loc}}^{2}$.

Proof. Since $V \in L_{\text {loc }}^{1}, \Delta f \in L_{\text {loc }}^{1}$. It is an easy result (see Lemma C.2.1), that $f \in L_{\text {loc }}^{\infty}, \Delta f \in L_{\text {loc }}^{1}$ implies $\nabla f \in L_{\text {loc }}^{2}$.

In $\S \mathrm{B} .6$, we will discuss the semigroup $e^{-t H}$ on the weighted $L^{2}$-spaces $L_{\delta}^{2}=\left\{f \mid\left(1+x^{2}\right)^{\delta / 2} f \in L^{2}\right\}$ for any $\delta$, positive or negative. We will prove then that

LemMA B.3.3. If $f \in L_{\delta}^{2}$, then $g \equiv e^{-t H} f$ is in $L_{\mathrm{loc}}^{\infty}$ and the distributional sum $-\frac{1}{2} \Delta g+V g$ lies in $L_{\text {loc }}^{\infty}$.

Theorem B.3.4. Let $V_{+} \in K_{\nu}^{\mathrm{loc}}, V_{-} \in K_{\nu}$. Let $f \in L^{p}$ for some $p \in[1, \infty]$. Then for any $t>0, e^{-t H} f$ has a distributional gradient in $L_{\text {loc }}^{2}$.

REMARKS. 1. The same proof actually shows that $f$ need only lie in $L f$ for some $\delta$.

2. In §B.6, we will show that if $f \in L^{\infty}$, then $(H-z)^{-1} f=g$ has a distributional gradient in $L_{\text {loc }}^{2}$.

ProOF. Since $e^{-t H} f=\left(e^{-t H / 2}\right)\left(e^{-t H / 2} f\right)$, we may suppose $p=\infty$. But $L^{\infty}$ $\subset L_{\delta}^{2}$ for any $\delta<\nu / 2$. Now use the last lemma and proposition.

Open problem. One would guess that if $\alpha>\frac{1}{2}+\nu(2 p)^{-1}$ then, for any $f \in L^{p},(H-z)^{-\alpha} f$ has a distributional gradient in $L_{\text {loc }}^{2}$. Prove it!

The example above also shows that eigenfunctions need not be Hölder continuous of any prescribed order. Thus, to obtain results on Hölder continuity of $\operatorname{Ran}\left(e^{-t H}\right)$ we need stronger hypotheses on $V$, a theme going back to a basic paper of Kato [110]; see also Simon [180].

Definition. Let $0<\alpha<2$. We define $K_{\nu}^{(\alpha)}$ as follows:

(1) When $\nu=1$ and $\alpha \leqslant 1, K_{\nu}^{(\alpha)} \equiv K_{\nu}$.

(2) When $\alpha<1$ and $\nu \geqslant 2$ or $\alpha>1, V \in K_{\nu}^{(\alpha)}$ if and only if

$$
\sup _{x} \int_{|x-y| \leqslant 1}|x-y|^{-(\nu-2+\alpha)}|V(y)| d y<\infty \text {. }
$$

(3) When $\alpha=1, \nu \geqslant 2, V \in K_{\nu}^{(\alpha)}$ if and only if

$$
\lim _{r \downarrow 0} \sup _{x} \int_{|x-y| \leqslant r}|x-y|^{-(\nu-1)}|V(y)| d y=0 .
$$


REMARKS. 1. Thus for $\alpha \neq 1, \nu \geqslant 2, K_{\nu}^{(\alpha)}$ is identical to the Schechter space $M_{2-\alpha, 1}$.

2. By arguments similar to those in Chapter A, $K_{\nu}^{(\alpha)} \subset K_{\mu}^{(\alpha)}$ if $\nu \leqslant \mu$; $L_{u}^{p} \subset K_{\nu}^{(\alpha)}$ so long as $p>\nu /(2-\alpha)$. Coulomb potentials lie in $K_{\nu}^{(\alpha)}$ if $\alpha<1$ but not in $K_{\nu}^{(1)}$. Since eigenfunctions are not in $C^{1}$ in that case (see $[110,89]$ for detailed analysis) this is not coincidental.

Definition. Let $0<\alpha<2$. $f \in C_{\alpha}(\Omega), \Omega$ is an open set of $R^{\nu}$ if and only if

(1) if $\alpha<1$, for every compact $K$ in $\Omega$, there is a $C$ with $|f(x)-f(y)| \leqslant C$ $|x-y|^{\alpha}$ for all $x, y \in K$;

(2) if $\alpha=1, f$ is $C^{1}$ on $\Omega$;

(3) if $1<\alpha<2, f$ is $C^{1}$ and $\nabla f$ is in $C_{\alpha-1}$.

In the remainder of this section, we will prove

THEOREM B.3.5. Let $V_{-} \in K_{\nu}, V_{+} \in K_{\nu}^{\text {loc }}$. Suppose that the restriction of $V$ to some bounded open set $\Omega$ lies in $K_{\nu}^{(\alpha)}$. Let $f \in \cup_{p} L^{p}\left(R^{\nu}\right)$. Then, for each $t>0$, $e^{-t H_{f}}$ lies in $C_{\alpha}(\Omega)$.

LEMMA B.3.6. Let $\nu \geqslant 3$. Let $\mathfrak{X}$ be the characteristic function of a bounded open set and suppose $\mathfrak{X} V \in K_{\nu}^{(\alpha)}$. Then $(\Delta)^{-1} \mathfrak{X V}$ is a bounded map from $L^{\infty}$ to $C_{\alpha}\left(R^{\nu}\right)$.

Proof. First we consider $\alpha<1$. By interpolating between

$$
\left|a^{-(\nu-2)}-b^{-(\nu-2)}\right| \leqslant a^{-(\nu-2)}+b^{-(\nu-2)}
$$

and

$$
\left|a^{-(\nu-2)}-b^{-(\nu-2)}\right| \leqslant C|a-b|\left[a^{-(\nu-1)}+b^{-(\nu-1)}\right]
$$

for $a, b>0$ and using

$$
|| x-y|-| z-y|| \leqslant|x-z|
$$

for $x, y, z \in R^{\nu}$ we see that for $0<\alpha<1$

$$
\begin{aligned}
|| x-\left.y\right|^{-(\nu-2)} & -|z-y|^{-(\nu-2)} \mid \\
& \leqslant C|x-z|^{\alpha}\left[|x-y|^{-(\nu-2+\alpha)}+|y-z|^{-(\nu-2+\alpha)}\right] .
\end{aligned}
$$

This inequality immediately yields the required result.

The $\alpha=1$ case follows by computing the integral kernel of $\nabla(\Delta)^{-1} \mathcal{X V}$ and following the argument which proves Proposition A.2.4(c) [9]. Given the formula for $\nabla(\Delta)^{-1} \mathcal{X} V$, the fact that this map is into $C_{\alpha-1}$ when $\alpha>1$ is identical to the above proof.

Proof OF TheORem B.3.5. Since $e^{-t H / 2}$ maps $L^{p}$ to $L^{\infty}$, we can suppose $f \in L^{\infty}$ without loss. Since $K_{\nu}^{(\alpha)} \subset K_{\mu}^{(\alpha)}$ if $\mu>\nu$, we can suppose $\nu \geqslant 3$. Let $g=e^{-t H} f$ and let $h$ be the distributional sum $-\Delta g+2 V g$ which lies in $L_{\text {loc }}^{\infty}$ by Lemma B.3.3. Let $\mathcal{X}$ be the characteristic function of $\Omega$, and let

$$
\tilde{g}=(-\Delta)^{-1} \mathfrak{X}\{h-2 V g\} \text {. }
$$

By Lemma B.3.6, $\tilde{g}$ lies in $C_{\alpha}$. Moreover, on $\Omega, \Delta(\tilde{g}-g)=0$ so $\tilde{g}-g$ is harmonic on $\Omega$ and thus $C^{\infty}$ there (see e.g. [161, §IX.6]). 
B4. Localization. It is no accident that we could not prove that $(H-z)^{-\alpha}$ is bounded from $L^{1}$ to $L^{\infty}$ when $\alpha<\nu / 2$; even when $V=0$, it is not-for if it were $\left(H_{0}-z\right)^{-\alpha}$ would have a bounded integral kernel. But the integral kernel $G_{0}^{(\alpha)}(x, y ; z)$ diverges as $c|x-y|^{-\lambda}$ near $x=y$ where

$$
\lambda=\nu-2 \alpha \text {. }
$$

But, at least if $V=0$, the divergence is only on diagonal and so $f\left(H_{0}-z\right)^{-\alpha} g$ is bounded from $L^{2}$ to $L^{\infty}$ if $f$ and $g$ have disjoint supports. In this section, we will prove this for $V \neq 0$ with estimates so good later they will control the divergence on the diagonal by the right power.

The estimate which will yield all others is

LEMMA B.4.1. Let $f, g$ be bounded functions. Then for any $p \leqslant q$

$$
\left\|f e^{-t H} g\right\|_{p, q} \leqslant\left\|f e^{-t\left(H_{0}+2 V\right)} g\right\|_{p, q}^{1 / 2}\left\|f e^{-t H_{0}} g\right\|_{p, q}^{1 / 2} \text {. }
$$

Proof. By the Schwarz inequality in function space (similar to (B8))

$$
\left|\left(e^{-t H} h\right)(x)\right| \leqslant\left[\left(e^{-t\left(H_{0}+2 V\right)}|h|\right)(x)\right]^{1 / 2}\left[\left(e^{-t H_{0}}|h|\right)(x)\right]^{1 / 2} .
$$

Multiplying by $f(x)$, setting $h=g \psi$ and maximizing over $\psi$, we obtain (B16).

Proposition B.4.2. Fix $V$ with $V_{+} \in K_{\nu}^{\text {loc }}, V_{-} \in K_{\nu}$. Let $\mathfrak{X}_{1}$ and $\mathfrak{X}_{2}$ be the characteristic function of two disjoint sets a distance $d>0$ apart. Then for all $0<t<1$

$$
\left\|X_{1} e^{-t H} X_{2}\right\|_{1, \infty} \leqslant C t^{-\nu / 2} e^{-d^{2} / 4 t}
$$

where $C$ depends only on $V$.

Proof. Use (B16) with $p=1, q=\infty$. The first factor is bounded by $\left(\tilde{C} t^{-\nu / 2}\right)^{1 / 2}$ by using (B11). The second is bounded by

$$
\left[(2 \pi t)^{-\nu / 2} \exp \left(-d^{2} / 2 t\right)\right]^{1 / 2}
$$

by the explicit kernel for $e^{-t H_{0}}$.

REMARK. By using the Riesz-Thorin theorem and the boundedness of $\left\|e^{-t H}\right\|_{p, p}$ uniformly in small time, we find that for $p \leqslant q$

$$
\left\|X_{1} e^{-t H} \mathcal{X}_{2}\right\|_{p, q} \leqslant C\left(t^{-\nu / 2} e^{-d^{2} / 4 t}\right)^{K}
$$

with $K=p^{-1}-q^{-1}$.

TheOREM B.4.3. Fix $V$ with $V_{+} \in K_{\nu}^{\text {loc }}, V_{-} \in K_{\nu}$ and $0<\alpha<\nu / 2$. Let $\mathcal{X}_{1}, \mathcal{X}_{2}$ be the characteristic function of two sets a distance $d>0$ apart. Let $\operatorname{Re} z<\inf \operatorname{spec}(H)$. Then $\mathcal{X}_{1}(H-z)^{-\alpha} \mathcal{X}_{2}$ is bounded from $L^{1}$ to $L^{\infty}$ with

$$
\left\|\mathscr{X}_{1}(H-z)^{-\alpha} \mathscr{X}_{2}\right\|_{1, \infty} \leqslant C[\min (d, 1)]^{-\lambda}
$$

where $C$ only depends on $V, z, \alpha$ and $\lambda$ is given by (B15). 
PROOF. $\int_{1}^{\infty} t^{\alpha-1} e^{2 t}\left\|e^{-t H}\right\|_{1, \infty}$ is bounded by $\S \mathrm{B} 1$. We bound

$$
\int_{0}^{1} t^{\alpha-1} e^{z t}\left\|\mathcal{X}_{1} e^{-t H} X_{2}\right\|_{1, \infty} d t
$$

by (using Proposition B.4.2)

$$
D \int_{0}^{\infty} t^{-\lambda / 2-1} e^{-d^{2} / 2 t} d t=C d^{-\lambda}
$$

by scaling and the fact that

$$
\int_{0}^{\infty} s^{-\lambda / 2-1} e^{-1 / 2 s} d s<\infty \quad \text { if } \lambda>0 .
$$

B5. Growth of $L^{p}$-semigroup norms as $t \rightarrow \infty$. In this section, we briefly summarize results on the large $t$ behavior of $\left\|e^{-t H}\right\|_{p, p}$ obtained in [68, 189, 191]. The first result implies the $p$ independence of

$$
\inf \operatorname{spec}(H)\left(=-\lim _{t \rightarrow \infty} t^{-1} \ln \left\|e^{-t H}\right\|_{p, p}\right) .
$$

ThEOREM B.5.1 [189]. $\lim _{t \rightarrow \infty} t^{-1} \ln \left\|e^{-t H}\right\|_{p, p}$ is $p$ independent. In fact, for any $p$

$$
\left\|e^{-t H}\right\|_{2,2} \leqslant\left\|e^{-t H}\right\|_{p, p} \leqslant\left\|e^{-t H}\right\|_{\infty, \infty}
$$

and

$$
\left\|e^{-t H}\right\|_{\infty, \infty} \leqslant C(1+t)^{\nu / 2}\left\|e^{-t H}\right\|_{2,2}
$$

where $C$ depends on $V$.

SKETCH. (B19) is immediate from duality and interpolation and (B19)-(B20) imply the first assertion. Thus we only need (B20). To prove this, let $f_{x, R}$ be the characteristic function of the ball of radius $R$ about $x$ and let $g_{x, R}=1-f_{x, R}$. Then

$$
\left|\left(e^{-t H} 1\right)(x)\right| \leqslant\left|\left(e^{-t H} f_{x, R}\right)(x)\right|+\left|\left(e^{-t H} g_{x, R}\right)(x)\right| .
$$

Let $\alpha_{2}=-\ln \left\|e^{-H}\right\|_{2,2}$ so, by the spectral theorem, $\left\|e^{-t H}\right\|_{2,2}=e^{t \alpha_{2}}$. Clearly for $t \geqslant 1$

$$
\left|\left(e^{-t H} f_{x, R}\right)(x)\right| \leqslant\left\|e^{-t H} f_{x, R}\right\|_{\infty} \leqslant\left\|e^{-H}\right\|_{2, \infty} e^{-t\left(\alpha_{2}-1\right)}\left\|f_{x, R}\right\|_{2}=C_{1} e^{-t \alpha_{2}} R^{\nu / 2} .
$$

By the estimate in Lemma B.4.3, for $t \geqslant 1$,

$$
\begin{aligned}
& \left|\left(e^{-t H} g_{x, R}\right)(x)\right| \leqslant\left\|e^{-t\left(H_{0}+2 V\right)}\right\|_{1,1}^{1 / 2}\left|\left(e^{-t H_{0}} g_{x, R}\right)(x)\right|^{1 / 2} \\
& \leqslant C e^{A t} e^{-D R^{2} / t}
\end{aligned}
$$

for suitable $A, D$ (using Theorem B.1.1 and the explicit kernel for $e^{-t H_{0}}$ ). Choosing $R=K t$ with $K$ large, we have (B20).

REMARKS. 1. In [189], (B21) is proven using more involved path integral properties.

2. In [189], it is proven that for any $p \leqslant q, \lim _{t \rightarrow \infty} t^{-1} \ln \left\|e^{-t H}\right\|_{p, q}$ is independent of $p, q$ and in [191], that one gets the same limit by taking any nonnegative $f \neq 0$ and looking at $\lim _{t \rightarrow \infty} t^{-1} \ln \left\|e^{-t H} f\right\|_{\infty}$ or even $\lim _{t \rightarrow \infty} t^{-1} \ln$ $\left|\left(e^{-t H} f\right)(x)\right|$ at any $x$. 
The $p$-independence of inf $\sigma(H)$ raises an

Open question. Prove that the $L^{p}$-spectrum of $H$ is $p$ independent.

By interpolation and duality one has that for $2 \leqslant p \leqslant r, \operatorname{spec}_{L^{2}}(H) \subset$ $\operatorname{spec}_{L^{p}}(H) \subset \operatorname{spec}_{L^{r}}(H)$.

One question of detailed interest is whether $e^{-\alpha_{2} t}\left\|e^{-t H}\right\|_{\infty, \infty}$ is bounded as $t \rightarrow \infty$. By (B20) the growth cannot be worse than $t^{\nu / 2}$. The main result known is for a very restricted class.

TheOREM B.5.2 $[189,191]$. Let $\nu \geqslant 3$. Fix $V \leqslant 0$ with $V \in L^{p} \cap L^{q}$ for some $p<\nu / 2<q$. Let $\alpha_{2}(\lambda)$ denote $\alpha_{2}$ for $H_{0}+\lambda V$. Then

(i) If $\alpha_{2}(1)>0$, then

$$
\lim _{t \rightarrow \infty} e^{-\alpha_{2} t}\left\|e^{-t H}\right\|_{\infty, \infty}
$$

exists and is a nonzero finite number.

(ii) If $\alpha_{2}(\lambda)=0$ for some $\lambda>1$, then

$$
\lim _{t \rightarrow \infty}\left\|e^{-t H}\right\|_{\infty, \infty}
$$

exists and is a nonzero finite number.

(iii) If $\alpha_{2}(1)=0$, but $\alpha_{2}(\lambda)>0$ for every $\lambda>1$, then

$$
\lim _{t \rightarrow \infty} g(t)^{-1}\left\|e^{-t H}\right\|_{\infty, \infty}
$$

exists and is a nonzero, finite number, where

$$
\begin{aligned}
g(t) & =t^{1 / 2} & & (\nu=3) \\
& =t / \ln t & & (\nu=4) \\
& =t & & (\nu=5) .
\end{aligned}
$$

There are a host of open questions. $t$ ?

Open questions. 1. Is the growth of $e^{-\alpha_{2} t}\left\|e^{-t H}\right\|_{\infty, \infty}$ ever worse than linear in

2. If so, is it as bad as $t^{\nu / 2}$ ?

3. If not, is there a dimension independent bound on the growth?

4. Find some examples which are not covered by Theorem B.5.2 where the explicit large $t$ behavior is computable. $\S \mathrm{C} 8$.

One theme of [189] is to connect divergence of $\left\|e^{-t H}\right\|_{\infty, \infty}$ to the subject of

THEOREM B.5.3. Let $V \in K_{\nu}, V \leqslant 0$. Then $\sup _{t}\left\|e^{-t H}\right\|_{\infty, \infty}<\infty$ if and only if there exists $\eta \in L^{\infty}$ with (i) $e^{-t H} \eta=\eta$; (ii) $\inf _{x} \eta(x)>0$.

Remarks. 1. Because $V \leqslant 0$, if $\nu=1,2, \alpha_{2}$ is never 0 so this result is of minimal interest there. Similarly, if $V$ falls off any slower than $|x|^{-2}, \alpha_{2}$ is not zero. Thus, the result is mainly of interest when $V \in L^{p} \cap L^{q}$ as it is stated in [189]. If $V \leqslant 0$ is dropped, it can happen that $\alpha_{2}=0$.

2. Let $\eta_{+}=\sup _{x} \eta(x) ; \eta_{-}=\inf _{x} \eta(x)$. Then [189] $\sup _{t}\left\|e^{-t H}\right\|_{\infty, \infty}=$ $\eta_{+} / \eta_{-}$.

Open question. What is the general relation between $\sup _{t} e^{-\alpha_{2} t}\left\|e^{-t H}\right\|_{\infty, \infty}<$ $\infty$ and existence of solutions of $H \eta=-\alpha_{2} \eta$ in $L^{\infty}$ ?

For $\nu=1$, this problem is partially addressed in [191]. 
B6. Weighted $L^{2}$-spaces. Here we will study $L^{p}$-properties of $w e^{-t H} w^{-1}$ for suitable weight functions. We concentrate on the polynomial weight case, $w=\left(1+x^{2}\right)^{\delta / 2}$ with $\delta$ real (positive or negative). Almost identical results (except for Theorem B.6.4) hold for the exponential weight case $w=e^{a|x|}(a$ real), with very similar proofs.

These results have two consequences. First, when $w$ is chosen to go to infinity at infinity (e.g. $\delta>0$ ), then we can define $e^{-t H} f$ for $f$ growing at infinity, e.g. if $w^{-1} f \in L^{\infty}$. When $w$ is chosen to decay at infinity (e.g. $\delta<0$ ), we obtain information telling us that if $f$ vanishes at infinity at some rate, then so does $e^{-t H} f$.

Rather than explicitly say that $w e^{-t H} w^{-1}$ is bounded from $L^{p}$ to $L^{q}$, it is more usual to introduce the spaces $w^{-1} L^{p}$ and $w^{-1} L^{q}$ and say that $e^{-t H}$ is bounded from $w^{-1} L^{p}$ to $w^{-1} L^{q}$. With this in mind, we define

Definition. $L p=\left\{f \mid\left(1+x^{2}\right)^{\delta / 2} f \in L^{p}\right\}$ with $\|f\|_{p, \delta}=\left\|\left(1+x^{2}\right)^{\delta / 2} f\right\|_{p}$. $\|A\|_{p, \delta ; q, \delta^{\prime}}$ is the norm of a map $A$ from $L_{\delta}^{p}$ to $L_{\delta}^{q}$.

LEMMA B.6.1. For any $p \leqslant q, e^{-t H_{0}}$ is bounded from $L_{\delta}^{p}$ to $L_{\delta}^{q}$ and

$$
\left\|e^{-t H_{0}}\right\|_{p, \delta ; q, \delta} \leqslant C_{p, q, \delta}\left[1+t^{|\delta| / 2}\right] t^{-\gamma}
$$

with $\gamma$ given by $\left(\mathrm{B} 11^{\prime}\right)$.

Proof. Let $K_{\delta}(x, y ; t)$ be the integral kernel of $\left(1+x^{2}\right)^{\delta / 2} e^{-t H_{0}}$ $\left(1+x^{2}\right)^{-\delta / 2}$

$$
K_{\delta}(x, y ; t)=\left(1+x^{2}\right)^{\delta / 2}(2 \pi t)^{-\nu / 2} e^{-(x-y)^{2} / 2 t}\left(1+y^{2}\right)^{-\delta / 2} .
$$

If $\delta \geqslant 0$, write

$$
\left(1+x^{2}\right)^{\delta / 2} \leqslant C\left[\left(1+y^{2}\right)^{\delta / 2}+|y-x|^{\delta}\right]
$$

and find

$$
\left|K_{\delta}(x, y ; t)\right| \leqslant C K_{0}(x, y ; t)+C|y-x|^{|\delta|} K_{0}(x, y ; t) .
$$

An identical estimate holds if $\delta<0$ (note the $|\delta|$ ). Since each term on the right of (B21) is a convolution kernel, one can estimate norms by Young's inequality.

LEMMA B.6.2. (a) Let $f \in L f$ for $q \geqslant p \geqslant 2$. Then

$$
\left\|e^{-t H} f\right\|_{q, \delta} \leqslant\left\|e^{-t\left(H_{0}+2 V\right)} 1\right\|_{\infty}^{1 / 2}\left\|e^{-t H_{0}}\right\|_{q / 2,2 \delta ; p / 2,2 \delta}^{1 / 2}\|f\|_{p, \delta} .
$$

(b) For any $p, q$

$$
\left\|e^{-t H}\right\|_{p, \delta ; q, \delta} \leqslant\left\|e^{-t\left(H_{0}+2 V\right)}\right\|_{p, q}^{1 / 2}\left\|e^{-t H_{0}}\right\|_{p, 2 \delta ; q, 2 \delta}^{1 / 2}
$$

Proof. (a) Follows from (B8).

(b) Follows from the extension of (B17) where the first $h$ on the right is replaced by $\left(1+x^{2}\right)^{\delta / 2}|h|$ and the second by $\left(1+x^{2}\right)^{-\delta / 2}|h|$. 
TheOREM B.6.3. Fix $1 \leqslant p<q \leqslant \infty$ and $\delta$ real. Then $e^{-t H}$ is bounded from $L_{\delta}^{p}$ to $L_{\delta}^{q}$ and

$$
\left\|e^{-t H}\right\|_{p, \delta ; q, \delta} \leqslant C t^{-\gamma} e^{A t}
$$

with $\gamma$ given by $\left(\mathrm{B} 11^{\prime}\right)$. In particular, if $\delta$ is fixed and if $w$ is sufficiently negative $(H-w)^{-\alpha}$ maps $L p$ to $L_{\delta}^{q}$ if $p^{-1}-q^{-1}<2 \alpha / \nu$.

First Proof. By Lemmas B.6.1 and B.6.2(a), we obtain the result for $q \geqslant p \geqslant 2$. By duality (since we have the result for $\delta$ and $-\delta$ ), we get the result for $p \leqslant q \leqslant 2$. Use the semigroup property if $p \leqslant 2 \leqslant q$.

SeCond Proof. Use Lemma B.6.1, Lemma 6.2(b) and Theorem B.1.1.

The following is proven in [190]; an alternate proof appears at the end of the section.

THEOREM B.6.4 [190]. Let $H_{\delta}$ denote the generator of the semigroup $e^{-t H}$ on $L_{\delta}^{2}$. Then the spectrum of $H_{\delta}$ is independent of $\delta$.

REMARK. Even if $V=0$, this result does not extend to the exponentially weighted spaces.

The following will be needed in $\S \mathrm{B} 12$; since $L^{\infty} \subset L_{-\delta}^{2}$ for some $\delta>0$ it also shows that if $f \in L^{\infty}$, then $(H-z)^{-1} f$ has a distributional gradient in $L_{\text {loc }}^{2}$.

TheOREM B.6.5. Let $V_{+} \in K_{\nu}^{\text {loc }}, V_{-} \in K_{\nu}$. Then

$$
p\left(1+x^{2}\right)^{\delta / 2}(H-z)^{-1}\left(1+x^{2}\right)^{-\delta / 2}
$$

and

$$
\left(1+x^{2}\right)^{\delta / 2} p(H-z)^{-1}\left(1+x^{2}\right)^{-\delta / 2}
$$

are bounded on $L^{2}$ for any real $\delta$ where $p=i \nabla$. Moreover, for any $\delta$,

$$
\lim _{M \rightarrow \infty} \sup _{\operatorname{Re} z<-M}\left\|p\left(1+x^{2}\right)^{\delta / 2}(H-z)^{-1}\left(1+x^{2}\right)^{-\delta / 2}\right\|_{2,2}=0 .
$$

Proof. Since $\left[p,\left(1+\mathrm{x}^{2}\right)^{\delta / 2}\right.$ ] is a multiplication operator bounded by $\left(1+x^{2}\right)^{\delta / 2}$, the boundedness of the second operator follows from that of the first and Theorem B.6.3. By commuting $\left(1+x^{2}\right)^{\delta / 2}$ and $(H-z)^{-1}$ we see that

$$
p\left(1+x^{2}\right)^{\delta / 2}(H-z)^{-1}\left(1+x^{2}\right)^{-\delta / 2}=A+B+C
$$

where

$$
\begin{aligned}
& A=p(H-z)^{-1} \\
& B=-i p(H-z)^{-1} p \cdot\left\{\nabla\left(1+x^{2}\right)^{\delta / 2}\right\}(H-z)^{-1}\left(1+x^{2}\right)^{-\delta / 2}, \\
& C=\frac{1}{2} p(H-z)^{-1}\left[\Delta\left(1+x^{2}\right)^{\delta / 2}\right](H-z)^{-1}\left(1+x^{2}\right)^{-\delta / 2} .
\end{aligned}
$$

Since $\nabla\left(1+x^{2}\right)^{\delta / 2}$ and $\Delta\left(1+x^{2}\right)^{\delta / 2}$ are bounded by $c\left(1+x^{2}\right)^{\delta / 2}$ and $(H-z)^{-1}$ and $p(H-z)^{-1} p$ are bounded (since $\left.Q(H)=Q\left(H_{0}\right)\right), A, B, C$ are bounded by Theorem B.6.3. The proof of that theorem shows that

$$
\lim _{M \rightarrow \infty} \sup _{\operatorname{Re} z<-M}\left\|(H-z)^{-1}\right\|_{2, \delta ; 2, \delta}=0 .
$$


Also $p(H-z)^{-1}, p(H-z)^{-1} p$ are bounded uniformly in a suitable left half plane. This implies the last assertion.

We needed the following result in $\S \mathrm{B} 3$.

LEMMA B.6.6 (三 LEMMA B.3.3). If $f \in L_{\delta}^{2}$, then $g \equiv e^{-t H} f$ is in $L_{\mathrm{loc}}^{\infty}$ and the distributional sum $-\frac{1}{2} \Delta g+V g$ lies in $L_{\text {loc }}^{\infty}$.

Proof. First, we will find a function $h \in L_{\mathrm{loc}}^{\infty}$ which is formally $\mathrm{Hg}$ and then we will show that $h$ is the required distributional sum. Since $e^{-t H}$ is bounded and holomorphic in $z$ on $L^{2}$ for $\operatorname{Re} z>0$, and on all $L_{\delta}^{2}$ for $z>0$, the Stein interpolation theorem (see e.g. [161, §X.8]) implies that $e^{-t H}$ is bounded and holomorphic in $z$ on each $L_{\delta}^{2}$. Thus, $H e^{-t H / 2} f \in L_{\delta}^{2}$ so we can define $h=$ $e^{-t H / 2} H e^{-t H / 2} f$ in $L_{\delta}^{\infty} \subset L_{\mathrm{loc}}^{\infty}$. Now, pick $V_{n} \in C_{0}^{\infty}$ so that $e^{-t H_{n}} \rightarrow e^{-t H}$ strongly on each $L_{\delta}(p<\infty)$ and $V_{n} \rightarrow V$ in $K_{\nu}^{\text {loc }}$ (see $\left.\S \mathrm{B} 10\right)$. Let $h_{n}=e^{-t H_{n} / 2} H_{n} e^{-t H_{n} / 2}$ and $g_{n}=e^{-t H_{n}} f$. Since $g_{n} \rightarrow g$ in $L_{\delta}^{\infty}$ (see $\left.\S \mathrm{B} 10\right)$ for any $\varphi \in C_{0}^{\infty},((-\Delta+$ $\left.\left.V_{n}\right) \varphi, g_{n}\right) \rightarrow((-\Delta+V) \varphi, g)$. But since $V_{n} \in C_{0}^{\infty}$, it is easy to see that $((-\Delta+$ $\left.\left.V_{n}\right) \varphi, g_{n}\right)=\left(\varphi, h_{n}\right)$. Since $h_{n} \rightarrow h$ (use Vitali's theorem to deduce convergence of $H_{n} e^{-t H_{n}}$ from that of $\left.e^{-t H_{n}}\right)$, we have the required $((-\Delta+V) \varphi, g)=(\varphi, h)$.

As the next idea, we want to note that $e^{-t H} f$ can be defined for $f$ growing rather quickly at infinity.

Proposition B.6.7. Let $e^{-t H}(x, y)$ be the integral kernel of $e^{-t H}$. Then for any $\varepsilon>0$

$$
\left|e^{-t H}(x, y)\right| \leqslant C_{\varepsilon} t^{-\nu / 2} e^{A t} \exp \left[-(x-y)^{2} / 2(1+\varepsilon) t\right] .
$$

Proof. By Hölder's inequality in the Feynman-Kac formula

$$
\left|\left(e^{-t H} f\right)(x)\right| \leqslant\left[\left(e^{-\left[t H_{0}+p V\right)}|f|\right)(x)\right]^{1 / p}\left[e^{-t H_{0}}|f|(x)\right]^{1 / q},
$$

so letting $f$ approach a $\delta$ function (and exploiting continuity of the integral kernels; see the next section):

$$
e^{-t H}(x, y) \leqslant\left[e^{-t\left(H_{0}+p V\right)}(x, y)\right]^{1 / p}\left[e^{-t H_{0}}(x, y)\right]^{1 / q} .
$$

The proposition now follows from control of the $L^{1} \rightarrow L^{\infty}$ norm of $e^{-t\left(H_{0}+p V\right)}$ (Theorem B.1.1) and the explicit form of $e^{-t H_{0}}(x, y)$.

The following are immediate from this proposition:

COROLLARY B.6.8. $e^{-t H} f$ can be defined (by an absolutely convergent integral or by requiring $\left(\varphi, e^{-t H} f\right)=\left(e^{-t H} \varphi, f\right)$ for all $\left.\varphi \in C_{0}^{\infty}\right)$ for all $t$ so long as

$$
|f(x)| \leqslant C_{a} e^{a x^{2}} \quad \text { for all } a>0 \text {. }
$$

COROLlARY B.6.9. If $f \in L^{1}$ has compact support, then

$$
\left|\left(e^{-t H} f\right)(x)\right| \leqslant C_{\varepsilon, f} t^{-v / 2} \exp \left(-x^{2} /(2+\varepsilon) t\right)
$$

for all $t>0$.

Here is a sketch of an alternate proof of Theorem B.6.4.

Lemma B.6.10. Let $V_{-} \in K_{\nu}, V_{+} \in K_{\nu}^{\text {loc }}$. Let $f \in C_{0}^{\infty}$. Then $p f(H-i)^{-1}$ is compact. 
Proof. Since $p(H+E)^{-1 / 2}$ is bounded, it suffices to show that

$$
(H+E)^{1 / 2} f(H-i)^{-1}
$$

is compact. This follows by interpolation from the fact that $f(H-i)^{-1}$ is compact (since $f\left(p^{2}+1\right)^{-1 / 2}$ is compact and $p(H-i)^{-1}$ is bounded) and that $H f(H-i)^{-1}=f H(H-i)^{-1}+2[p, f] p(H-i)^{-1}+[p,[p, f]](H-i)^{-1}$ is bounded.

Sketch of A Proof of Theorem B.6.4. Let $\rho=\left(1+x^{2}\right)^{1 / 2}$. Let $\vec{g}=\rho^{-1} \nabla \rho$ and

$$
H(\alpha)=\frac{1}{2}(\vec{p}-i \alpha \vec{g})^{2}+V .
$$

By the above lemma and a limiting argument $[H(\alpha)-H(0)](H+i)^{-1}$ is compact. Thus $\sigma_{\text {ess }}(H(\alpha))=\sigma_{\text {ess }}(H)$. For $\alpha$ purely imaginary $H(\alpha)=\rho^{\alpha} H \rho^{-\alpha}$ and so by the Combes-Thomas argument [43], $\sigma_{\text {disc }}(H(\alpha))=\sigma_{\text {disc }}(H)$. Note that $H(\alpha)$ is unitarily equivalent to $H$ on $L_{\alpha}^{2}$.

B7. Integral kernels: General potentials. Our goal in this section is to prove the following theorems, each of which is partly new.

THEOREM B.7.1. Let $V_{+} \in K_{\nu}^{\text {loc }}, V_{-} \in K_{\nu}$. Then the following operators are integral operators with jointly continuous, uniformly bounded integral kernels:

(a) $e^{-t H}$;

(b) $(H-z)^{-\alpha}$ where $\alpha>\nu / 2 ; \operatorname{Re} z<\inf \operatorname{spec}(H)$;

(c) $(H-z)^{-\alpha}$ where $\alpha>\nu / 2, \alpha$ an integer; $z \notin \operatorname{spec}_{L^{2}}(H)$;

(d) $f(H)$; f a Borel function on $\operatorname{spec}_{L^{2}}(H)$, obeying

$$
|f(x)| \leqslant C(1+|x|)^{-\alpha} ; \quad \alpha>\nu / 2 ; x \in \operatorname{spec}_{L^{2}}(H) .
$$

Moreover,

(a') For all $\varepsilon>0$

$$
\left|e^{-t H}(x, y)\right| \leqslant C_{\varepsilon}(t) \exp \left(-(x-y)^{2} / 2(1+\varepsilon) t\right) ;
$$

$\left(\mathrm{b}^{\prime}, \mathrm{c}^{\prime}\right)\left|(H-z)^{-\alpha}(x, y)\right| \leqslant C_{\alpha, \delta} \exp (-\delta|x-y|)$ for some $\delta>0$ and if $\operatorname{Re} z$ $<\Sigma=\inf \operatorname{spec}(H)$, for all $\delta$ obeying $\frac{1}{2} \delta^{2}<\Sigma-\operatorname{Re} z$.

$\left(\mathrm{a}^{\prime \prime}\right)\left|e^{-t H}(x, y)\right| \geqslant \tilde{C}_{\varepsilon}(t) \exp \left(-(x-y)^{2} / 2(1-\varepsilon) t\right)$ if $V_{+} \in K_{\nu}$;

$\left(\mathrm{a}^{\prime \prime \prime}\right) e^{-t H}(x, y)$ is jointly continuous in $x, y, t$ in the region $t>0$.

TheOREM B.7.2. Suppose $V_{+} \in K_{\nu}^{\text {loc }} V_{-} \in K_{\nu}$. Let $0<\alpha<\nu / 2$ and either $\operatorname{Re} z<\Sigma=\inf \operatorname{spec}(H)$ or $z \notin \operatorname{spec}_{L^{2}}(H)$, $\alpha$ integral. Then $(H-z)^{-\alpha}$ is an integral operator with integral kernel, $G^{(\alpha)}(x, y ; z)$, obeying

(1) $G$ is continuous away from $x=y$ and bounded uniformly in each region $\{(x, y)|| x-y \mid \geqslant d\}$.

(2) $\left|G^{(\alpha)}(x, y ; z)\right| \leqslant C|x-y|^{-\lambda} ; \lambda$ given by $(\mathrm{B} 15)$.

(3) For $|x-y|$ sufficiently small and $|x|<R$

$$
\left|G^{(\alpha)}(x, y ; z)\right| \geqslant \tilde{C}_{R}|x-y|^{-\lambda} ; \quad \lambda \text { given by }(\mathrm{B} 15) .
$$

If $V \in K_{\nu}, \tilde{C}_{R}$ can be chosen independent of $R$.

(4) For $|x-y| \geqslant 1$

$$
\left|G^{(\alpha)}(x, y ; z)\right| \leqslant C_{\delta, \alpha, z} \exp (-\delta|x-y|)
$$

for some $\delta>0$ and if $\operatorname{Re} z<\Sigma$, for all $\delta$ with $\frac{1}{2} \delta^{2}<\Sigma-\operatorname{Re} z$. 
REMARKs. 1. There are previous results (e.g. [83, 194]) implying that $e^{-t H}$ has a bounded integral kernel in great generality.

2. Among functions $f$ for Theorem B.7.1, of especial interest is the fact that the spectral projections of $H$ are integral operators.

3. Looking at the case $V=0$ shows that the integral kernel of $f(H)$ will not have rapid decay as $|x-y| \rightarrow \infty$ if $f$ is not smooth. If $f$ is in $C_{0}^{\infty}$, one can show that $|f(H)(x, y)| \leqslant C(|y|+1) /(|x|+1)$ as follows: one need only show $f$ is bounded from $L_{\delta=1}^{1}$ to $L_{\delta=1}^{\infty}$. By writing $f(H)=(H+i)^{-n} g(H)(H+i)^{-n}$ as usual, we only need boundedness from $L_{1}^{2}$ to $L_{1}^{2}$. Thus $L^{2}$-boundedness of $(|x|+1)^{-1}[x, f(H)]$ will suffice. But (following [151]) we have that

$$
[x, f(H)]=(2 \pi)^{-1 / 2} \int \hat{f}(\lambda)\left[x, e^{i \lambda H}\right]
$$

and

$$
e^{i \lambda H} x e^{-i \lambda H}=x+\int_{0}^{\lambda} d \mu e^{i \mu H} p e^{-i \mu H}
$$

so

$$
\left\|(|x|+1)^{-1}\left[x, e^{i \lambda H}\right](H+i)^{-1}\right\| \leqslant C(1+|\lambda|)
$$

from which

$$
(|x|+1)^{-1}[x, f(H)](H+i)^{-1}
$$

is bounded on $L^{2}$. Borrowing extra factors from $f$ (see [1511), one can complete the proof.

Open question. For $f \in C_{0}^{\infty}$, show that

$$
|f(H)(x, y)| \leqslant C_{n}(|x-y|+1)^{-n} .
$$

To begin the proof of these theorems, we note that we already know the following

LEMMA B.7.3. $e^{-t H}$ is an integral operator with integral kernel in $L^{\infty}$ and Theorem B.7.1( $\left.\mathrm{a}^{\prime}\right)$ holds.

Proof. The first assertion follows from Theorem B.1.1 and Corollary A.1.2. The second is Proposition B.6.6.

We will prove continuity of this integral kernel first for $V$ in $C_{0}^{\infty}$ and then for general $V$ by an approximation argument.

Lemma B.7.4. Let $V \in C_{0}^{\infty}$. Then $e^{-t H}(x, y)$ is jointly continuous in $x, y, t$.

Proof. Because of the bound $\left(\mathrm{a}^{\prime}\right)$ and the semigroup property, it suffices to prove the result for small $t$. We can choose $t$ so small that the series expansion in $t$ converges uniformly in $x, y$ and $t$ small by exploiting (B3). It thus suffices to prove joint continuity of $T_{n}(x, y ; t)$ where

$$
\begin{aligned}
T_{n}\left(x_{0}, x_{n} ; t\right)= & \int_{s_{1}+\cdots+s_{n}=t} d^{\nu} x_{1} \cdots d^{\nu} x_{n-1} d s_{1} \cdots d s_{n-1} \prod_{j=1}^{n-1} V\left(x_{j}\right) \\
& \cdot \prod_{j=1}^{n}\left(2 \pi s_{j}\right)^{-\nu / 2} \exp \left(-\left(x_{j}-x_{j-1}\right)^{2} / 2 s_{j}\right) .
\end{aligned}
$$


The change of variables

$$
\begin{aligned}
z_{j} & =\left[x_{j}-y_{j}\left(w_{i}\right)\right] / \sqrt{t}, \quad w_{i}=s_{i} / t ; \\
y_{j}\left(w_{i}\right) & =\left(w_{1}+\cdots+w_{j}\right)\left(x_{n}-x_{0}\right)+x_{0}
\end{aligned}
$$

(so $z_{0}=z_{n}=0$ ) yields

$$
\begin{aligned}
T_{n}\left(x_{0}, x_{n} ; t\right)= & t^{n} t^{-\nu / 2} \exp \left(-\left(x_{n}-x_{0}\right)^{2} / 2 t\right) \\
& \cdot \int_{w_{1}+\cdots+w_{n}=1} d^{\nu} z_{1} \cdots d^{\nu} z_{n-1} d w_{1} \cdots d w_{n-1} \\
& \cdot \prod_{j=1}^{n-1} V\left(z_{j} \sqrt{t}+y_{j}(w)\right) \prod_{j=1}^{n}\left(2 \pi w_{j}\right)^{-\nu / 2} \exp \left(-\left(z_{j}-z_{j-1}\right)^{2} / 2 w_{j}\right) .
\end{aligned}
$$

If $V$ is replaced by 1 , the integral converges (to $(2 \pi)^{-\nu / 2} / n$ !) so the joint continuity is an immediate consequence of the continuity of $V$ and the dominated convergence theorem.

REMARK. The change of variables (B23) which works so well is motivated by the fact [194] that if $b(s)$ is Brownian motion on $(0, t)$ conditioned to start at $x$ and end at $y$, then $t^{-1 / 2}\left(b(s / t)-t^{-1} s y-\left(1-s t^{-1}\right) x\right)$ is a process independent of $x, y, t$. Zelditch [207] uses a similar change of variables.

LEMMA B.7.5. $e^{-t H}(x, y)$ is jointly continuous in $x, y, t$ for general $V$ with $V_{+} \in K_{\nu}^{\text {loc }}, V_{-} \in K_{\nu}$.

PRoof. In $\S \mathrm{B} 10$, we will prove that for any such $V$, we can find $V_{n}$ in $C_{0}^{\infty}$ so that for any compact $K$,

$$
\lim _{n \rightarrow \infty}\left\|\mathscr{X}_{K}\left[e^{-t H}-e^{-t H_{n}}\right] \mathscr{X}_{K}\right\|_{1, \infty}=0
$$

uniformly as $t$ runs through compacts of $(0, \infty)$. It follows, by Theorem A.1.1, that $e^{-t H_{n}}(x, y)$ is uniformly convergent as $n \rightarrow \infty$ on compacts of $(x, y, t) \in$ $\mathbf{R}^{2 \nu} \times(0, \infty)$. Since uniform limits of continuous functions are continuous, we are done.

REMARK. There is another way of proving joint continuity in $(x, y)$. Let $s=$ $\frac{1}{3} t$ and note

$$
e^{-t H}(x, y)=\int e^{-s H}(x, z) e^{-s H}(y, w) e^{-s H}(z, w)
$$

since $e^{-s H}(y, w)=e^{-s H}(w, y)$. Let $\tilde{H}$ be the operator on $L^{2}\left(\mathbf{R}^{2 v}\right)$ given by $-\frac{1}{2} \Delta_{x}-\frac{1}{2} \Delta_{y}+V(x)+V(y)$. Then the above says that

$$
e^{-t H}(x, y)=\left(e^{-s \tilde{H}} f\right)(x, y)
$$

where $f(x, y)=e^{-s H}(x, y)$. Since $f \in L^{\infty}$, and $V_{+}(x)+V_{+}(y) \in K_{2 \nu}^{\text {loc }}$, etc. (since $K_{\nu} \subset K_{2 \nu}$ ), Theorem B.3.1 implies continuity of $e^{-t H}(x, y)$ on $R^{2 \nu}$.

LEMMA B.7.6. If $\operatorname{Re} z<\Sigma$ and $\alpha>0$, then $(H-z)^{-\alpha}$ has an operator with integral kernel which is continuous away from $x=y$, and

(a) for $\alpha>\nu / 2$ continuous for all $x, y$,

(b) for $\alpha<\nu / 2$, uniformly bounded on any set with $|x-y|>d$ and the bound Theorem B.7.2(2) holds. 
Proof. Since $(H-z)^{-\alpha}: L^{p} \rightarrow L^{\infty}$ for suitable $p<\infty$, it is an integral operator. By using the formula (A9) and the continuity of the integral kernel of $e^{-t H}(x, y)$, it suffices to get absolute convergence of the integrand. For $\alpha>\nu / 2$ this follows from (B11) and for $\alpha<\nu / 2$, we need only use Proposition B.4.2 to get the continuity away from $x=y$ and (B18) to get the required bound.

LEMMA B.7.7. For $z$ real and sufficiently negative, the lower bound Theorem B.7.2(3) holds.

Proof. Consider first the case $V \in K_{\nu}$. By the Schwarz inequality in function space:

$$
e^{-t H_{0}}(x, y) \leqslant\left[e^{-t\left(H_{0}+V\right)}(x, y)\right]^{1 / 2}\left[e^{-t\left(H_{0}-V\right)}(x, y)\right]^{1 / 2}
$$

so using the Schwarz inequality in (A9) we find

$$
\left(H_{0}-z\right)^{-\alpha}(x, y) \leqslant\left[(H-z)^{-\alpha}(x, y)\right]^{1 / 2}\left[\left(H_{0}-V-z\right)^{-\alpha}(x, y)\right]^{1 / 2}
$$

for $z$ sufficiently negative. Since $-V \in K_{\nu}$, we have an upper bound on $\left(H_{0}-V-z\right)^{-\alpha}(x, y)$ of the form $C|x-y|^{-\lambda}$ and by the explicit form of $\left(H_{0}-z\right)^{-\alpha}$ a lower bound on it by $\tilde{C}|x-y|^{-\lambda}$ from which the desired lower bound follows.

If $V_{+}$is only in $K_{\nu}^{\text {loc }}$ and we want to get the bound for $|x|,|y| \leqslant R$, we replace $H_{0}$ by $\tilde{H}_{0}$ the operator with Dirichlet boundary conditions on the cube of side $4 R$ centered at 0 and $V$ by $W=V \mathscr{X}$ with $\mathcal{X}$ the characteristic function of this cube. Then, as one proves (B24), one finds

$$
\left(\tilde{H}_{0}-z\right)^{-\alpha}(x, y)^{2} \leqslant\left(\tilde{H}_{0}+W-z\right)^{-\alpha}(x, y)\left(\tilde{H}_{0}-W-z\right)^{-\alpha}(x, y) .
$$

Since $e^{-t\left(\tilde{H}_{0}+W\right)}(x, y) \leqslant e^{-t\left(H_{0}+V\right)}(x, y)$ (see [194]) it follows that

$$
\left(\tilde{H}_{0}-z\right)^{-\alpha}(x, y)^{2} \leqslant\left(H_{0}+V-z\right)^{-\alpha}(x, y)\left(H_{0}-W-z\right)^{-\alpha}(x, y)
$$

which yields the required lower bound since $\left(\tilde{H}_{0}-z\right)^{-\alpha}$ can be seen to have a kernel diverging in the required way for $|x|,|y|<R$.

As preparation for the proof of Theorem B.7.1(d), we need

LeMma B.7.8. Let $C$ be a bounded operator on $L^{2}$ for which there exists a function $C(x, y)$ so that

(i) for each fixed $y, C(x, y) \in L^{2}$ and $y \mapsto C(x, y)$ is continuous in $L^{2}$-norm,

(ii) for $f \in L^{\infty}$ with compact support,

$$
(C f)(x)=\int C(x, y) f(y) d^{\nu} y .
$$

Let $A$ be an operator for which $A^{*}$ has a "kernel" with similar properties. Let $B$ be any bounded operator. Then there is a jointly continuous function $D(x, y)$ so that for $f, g \in L^{\infty}$ with compact support, we have

$$
(f, A B C g)=\int D(x, y) \overline{f(x)} g(y) d^{\nu} x d^{\nu} y .
$$


Proof. Let $C_{y}$ be the function $C(\cdot, y)$. (ii) says that $C g=\int g(y) C_{y} d^{\nu} y$ as an $L^{2}$-integral. Thus

$$
(f, A B C g)=\int g(y) \overline{f(x)}\left(A_{x}^{*}, B C_{y}\right) .
$$

By (i), $x, y \mapsto\left(A_{x}^{*}, B C_{y}\right)=D(x, y)$ is jointly continuous.

LEMMA B.7.9. Case (d) of Theorem B.7.1 is an integral operator with jointly continuous integral kernel.

Proof. By Theorem B.2.3 and Corollary A.1.2, $f(H)$ has an $L^{\infty}$ integral kernel. Writing $f(H)=(H-z)^{-\alpha / 2} g(H)(H-z)^{-\alpha / 2}$ with $-z$ large and $g(x)$ $=(x-z)^{\alpha} f(x)$, we can apply the last lemma if we note the bound we already have on the integral kernel of $(H-z)^{-\alpha / 2}$.

LemMa B.7.10. Let $\alpha$ be a positive integer, $\alpha<\nu / 2$. Let $z \notin \operatorname{spec}_{L^{2}}(H)$. Then $(H-z)^{-\alpha}$ is an integral operator obeying (1), (2), (3) of Theorem B.7.2.

Proof. Pick $w$ real and so negative that Lemmas B.7.6 and B.7.7 are applicable to $(H-w)^{-\alpha}$. Pick $k$ so large that $\alpha+k>\nu / 2$. Then we can expand

$$
(H-z)^{-\alpha}=\sum_{j=0}^{k-1}(-1)^{j}\left(\begin{array}{c}
\alpha+j-1 \\
j
\end{array}\right)(z-w)^{j}(H-w)^{-\alpha-j}+f(H)
$$

where $|f(x)| \leqslant C(1+|x|)^{-\alpha-k}$ on $\operatorname{spec}_{L^{2}}(H)$. Since $f(H)$ (by Lemma B.7.9) has a bounded integral kernel and we know all about the integral kernel of $(H-w)^{-\alpha-j}$ we can read off the required properties of $(H-z)^{-\alpha}$.

LEMMA B.7.11. For any $\alpha>0$, and $|x-y| \geqslant 1$,

$$
\left|G^{(\alpha)}(x, y ; z)\right| \leqslant C_{\delta, \alpha, z} \exp (-\delta|x-y|)
$$

where $\alpha$ is arbitrary if $\operatorname{Re} z<\Sigma$ and $\frac{1}{2} \delta^{2}<\Sigma$ and $\alpha$ is integral and $\delta$ some sufficiently small number if $z \notin \operatorname{spec}_{L^{2}}(H)$ with $\operatorname{Re} z \geqslant \Sigma$.

Remark. One can replace $\frac{1}{2} \delta^{2}<\Sigma$ by $\frac{1}{2} \delta^{2}<\Sigma_{(1)} \equiv \inf \operatorname{ess} \operatorname{spec}(H)$; see $\S \mathrm{C} 3$.

Proof. We combine the machinery used thus far with an idea of Combes and Thomas [43]. Fix $a \in R^{\nu}$ and let $H_{a}$ be the operator which is formally $e^{a \cdot x} \mathrm{He}^{-a \cdot x}$, i.e.

$$
\frac{1}{2}(i \nabla-i a)^{2}+V=H_{a} .
$$

Then $\operatorname{Re}\left(\varphi, H_{a} \varphi\right)=H-\frac{1}{2} a^{2}$. Since $Q(H)=Q(-\Delta)$, the $a \cdot \nabla$ term is $H$-form bounded in the sense of Kato [114] with relative bound zero, so $H_{a}$ is analytic and the spectrum of $H_{a}$ is continuous in $a$. It follows that, when $\operatorname{Re} z<\Sigma, z$ is not in $\operatorname{spec} \operatorname{Re}\left(H_{a}\right)$ if $\frac{1}{2}|a|^{2}<\Sigma-\operatorname{Re} z$ and if $z$ is only not in $\operatorname{spec}(H)$, then $z \notin \operatorname{spec}\left(H_{a}\right)$ for $|a|<\delta$ for small $\delta>0$. Using the explicit integral kernel of $\exp \left(-t\left(H_{a}-V\right)\right)$, one can show that $\left(H_{a}-z\right)^{-\alpha}$ has a bounded integral kernel by just mimicking our approach for $(H-z)^{-\alpha}$ so long as $z \notin \operatorname{spec}\left(H_{a}\right)$ [50]. 
But

$$
\left(H_{a}-z\right)^{-\alpha}(x, y)=\exp (a \cdot(x-y))(H-z)^{-\alpha}(x, y)
$$

so we conclude that for all $x, y$

$$
\left|(H-z)^{-\alpha}(x, y)\right| \leqslant C \exp (-a|x-y|)
$$

for all $a$ with $|a|^{2}<2(\Sigma-\operatorname{Re} z)$ or $|a|^{2}<\delta$. Since this holds for all directions, we obtain the required bound.

B8. Integral kernels: Some special operators for some special potentials. In this section, we want to consider integral kernels for $(H-z)^{-1}$ as $z$ approaches the continuous spectrum for $H$ and for $e^{-i t H}$. In neither case can one hope for results for all $V$ with $V_{+} \in K_{\nu}^{\text {loc }}, V_{-} \in K_{\nu}$ since existence of the first in a suitable sense implies the absence of singular continuous spectrum which can be false [149] and even for $H=-\Delta+x^{2}$ we have $e^{-i t H}=I$ for $t$ suitable and $I$ is certainly not an integral operator. We will therefore place restrictions on $V$ which are undoubtedly too strong.

THEOREM B.8.1. Let $(a, b)$ be an interval in $R$, let $V_{+} \in K_{\nu}^{\text {loc }}, V_{-} \in K_{\nu}$. Suppose that for some $\delta>0$, as operators from $L_{\delta}^{2}$ to $L_{-\delta}^{2},(H-z)^{-1}$ has a norm continuous extension from $S=\{z=\mu+i \lambda \mid 0<\lambda<1 ; \mu \in(a, b)\}$ to $\tilde{S}=$ $\{z=\mu+i \lambda \mid 0 \leqslant \lambda<1 ; \mu \in(a, b)\}$. Then the integral kernel $G(x, y ; z)$ of $(H-z)^{-1}$ also has a jointly continuous extension from $\left(R^{\nu} \times R^{\nu} \backslash \Delta\right) \times S$ to $\left(R^{\nu} \times R^{\nu} \backslash \Delta\right) \times \tilde{S}$, where $\Delta=\{(x, y) \mid x=y\}$.

Proof. We will show that for an integral operator $A(z)$ which has a continuous integral kernel on $\left(R^{\nu} \times R^{\nu} \backslash \Delta\right) \times \tilde{S},(H-z)^{-1}-A$ has a norm continuous extension from $S$ to $\tilde{S}$ as operators from $L_{\delta}^{1}$ to $L_{-\delta}^{\infty}$. It follows that $\left(1+|x|^{2}\right)^{-\delta}(G(x, y ; z)-A(x, y ; z))\left(1+|y|^{2}\right)^{-\delta}$ has such an extension in $L^{\infty}\left(R^{\nu} \times R^{\nu}\right)$-norm. Since $G$ is known to be continuous in $x, y$ when $\operatorname{Im} z>0$ (Theorem B.7.1), the theorem then follows.

We write $(H-z)^{-1}=A(z)+B(z)$ where

$$
A(z)=(H-w)^{-1}+(w-z)(H-w)^{-2}+\cdots+(w-z)^{k-1}(H-w)^{-k}
$$

where $k \geqslant 2$ is chosen so that $k>\nu / 2, w$ is very negative and

$$
B(z)=(w-z)^{k}(H-w)^{-k / 2}(H-z)^{-1}(H-w)^{-k / 2} \text {. }
$$

By the results in Theorem B.7.2, $A(x, y ; z)$ has the required joint continuity on $\left(R^{\nu} \times R^{\nu} \backslash 0\right) \times \tilde{S}$. By Theorem B.6.3, $(H-w)^{-k / 2}$ maps $L_{\delta}^{1}$ to $L_{\delta}^{2}$ and $L_{-\delta}^{2}$ to $L_{-\delta}^{\infty}$ so $B(z)$ has a norm continuous extension from $L_{\delta}^{1}$ to $L_{-\delta}^{\infty}$ as required.

REMARK. The proof shows that we have upper and lower bounds on $|G(x, y ; z)|$ by $C_{ \pm}|x-y|^{-(\nu-2)}$ for $|x-y|$ small, uniformly for $z \in \tilde{S}$.

There are three situations where we know that the hypothesis about $L_{\delta}^{2}$ to $L_{-\delta}^{2}$ extensions holds (all with arbitrary $\delta>\frac{1}{2}$ ).

(1) When $V$ is a "short range two-body potential", e.g. $V\left(1+|x|^{2}\right)^{1 / 2+\varepsilon} \in$ $L^{p}+L^{\infty}$ for some $\varepsilon>0$ and some $p>\nu / 2$, then the required estimates follow from the Agmon [1] and Kuroda [126] theory (see [162, §XIII.8] for an exposition) if $(a, b)$ avoids 0 and any eigenvalues (which are discrete away from zero). 
(2) The same estimates are known in various long range two body cases, e.g., if $V=V_{L}+V_{s}$ with $V_{s}$ obeying the hypothesis above and

$$
\left|D^{k} V_{L}(x)\right| \leqslant C_{k}(1+|x|)^{-k-\varepsilon}
$$

see $[3,102,103,104,117,168]$.

(3) For suitable $N$-body systems, such estimates are proven by Perry, Sigal and Simon [151].

For $e^{-i t H}$ the results are much weaker. Consideration of $V=0$ shows we cannot hope that they are integral operators in the sense of there being a kernel $K(x, y)$ with $\int|K(x, y)||f(y)| d y$ converging a.e. $x$ for every $f \in L^{2}$. We say that $A$ is a weak-integral operator with kernel $K(x, y)$ if and only if $K \in L_{\text {loc }}^{1}$ $\left(R^{2 \nu}\right)$ and for all $L^{\infty}$-functions of compact support $f, g$ we have

$$
(f, A g)=\int f(x) K(x, y) g(y) d^{\nu} x d^{\nu} y .
$$

THEOREM B.8.2. Suppose that $V$ is a $C^{\infty}$-function obeying

$$
\left|\left(D^{\alpha} V\right)(x)\right| \leqslant C_{\alpha}(1+|x|)^{k(\alpha)}
$$

for all $\alpha$ where either $k(\alpha)=k_{0}-|\alpha|$ with $k_{0}<2$ or $k(\alpha) \equiv 0$. Then $e^{-i t H}$ has a weak integral kernel $P(x, y ; t)$ for all $t \neq 0$ and it is jointly $C^{\infty}$ on $R^{\nu} \times R^{\nu} \times$ $(R \backslash\{0\})$.

This was proven by Fujiwara [61, 62] in a series of papers; see also Fujiwara [63], Kitada [118], Kitada and Kumanoago [119] and see Zelditch [207] for an alternative and simpler proof.

Zeldtich [207] also has results for $k_{0}=2$ when $|t|$ is sufficiently small and studies the case $k_{0}=2$ in detail for larger $t$ where, as the case $V=x^{2}$ shows, it can happen that $P$ stops existing for some larger $t$.

All previous results on $e^{-i t H}$ require $V$ to be smooth. The following new results illustrate that this is not necessary.

THEOREM B.8.3. Let $\nu=1$. Let $V \in L^{1}$. Then $e^{-i t H}$ is a weak integral operator for $t \neq 0$ with integral kernel $P(x, y ; t)$ which is jointly continuous in $(x, y, t)$ on $R \times R \times(R \backslash\{0\})$.

We begin the proof with

LEMMA B.8.4. Fix $0<\alpha<1$. Then

$$
\int_{s_{0}+\cdots+s_{n}=t} d s_{1} \cdots d s_{n}\left[\prod_{j=0}^{n} s_{j}^{-\alpha}\right] \leqslant \frac{D_{\alpha}^{n+1}}{(n !)^{1-\alpha}} t^{n(1-\alpha)-\alpha} .
$$

Proof. We evaluate exactly the LHS of (B25), call it $B_{n, \alpha}(t)$, using the same method that relates the beta function to the gamma function $(n=2)$. By scaling $B_{n, \alpha}(t)=B_{n, \alpha}(1) t^{n(1-\alpha)-\alpha}$. Thus

$$
\int_{0}^{\infty} B_{n, \alpha}(t) e^{-t} d t=B_{n, \alpha}(1) \Gamma((n+1)(1-\alpha)) .
$$


But

$$
\int_{0}^{\infty} B_{n, \alpha}(t) e^{-t} d t=\int \prod_{j=0}^{n}\left[s_{j}^{-\alpha} e^{-s_{j}} d s_{j}\right]=\Gamma(1-\alpha)^{n+1} .
$$

(B25) now follows from the asymptotics of $\Gamma(x)$ as $x \rightarrow \infty$.

Proof of Theorem B.8.3. Let $Q_{n}(x, y ; t, V)$ be defined for $n=1,2, \ldots$ by

$$
Q_{n}\left(x_{0}, x_{n} ; t, V\right)=\int_{s_{1}+\cdots+s_{n}=t}\left[\prod_{j=1}^{n-1} d s_{j} d x_{j}\right] \prod_{j=1}^{n} P_{0}\left(x_{j-1}, x_{j} ; s_{j}\right) \prod_{j=1}^{n-1} V\left(x_{j}\right)
$$

with $P_{0}(x, y ; s)=(2 \pi i s)^{-1 / 2} \exp \left(-(x-y)^{2} / 2 i s\right)$. By (B25) we obtain

$$
\begin{gathered}
\left|Q_{n}(x, y ; t, V)\right| \leqslant D^{n}(n !)^{-1 / 2}\|V\|_{1}^{n-1} t^{(n-2) / 2} \\
\left|Q_{n}(x, y ; t, V)-Q_{n}(x, y ; t, W)\right| \\
\leqslant n D^{n}(n !)^{-1 / 2} t^{(n-2) / 2}\left[\|V\|_{1}+\|W\|_{1}\right]^{n-2}\left[\|V-W\|_{1}\right] .
\end{gathered}
$$

Moreover, the change of variables (B23) shows that when $V \in C_{0}^{\infty}, Q_{n}$ is jointly continuous in $x, y, t$. By (B27), this joint continuity remains true for all $V \in L^{1}$ and so by (B26), $\sum_{n=1}^{\infty} Q_{n}(x, y ; t, V)$ is jointly continuous. It is easy to see that this sum is a weak integral kernel for $e^{-i t H}$.

The most important defect in the above theorem is the restriction to $\nu=1$. For $\nu \geqslant 2$, the integrals defining $Q_{n}$ are no longer absolutely convergent at $s_{j}=0$, but that should not change the situation except to require a stronger condition on $V\left(V \in L^{1}\right.$ does not imply $V \in K_{\nu}$ if $\left.\nu \geqslant 2\right)$ and a new proof. Moreover, the restriction at $\infty$ required by $V \in L^{1}$ is the wrong one.

Open question. Prove for a class of $N$-body Schrödinger operators including atomic Hamiltonians that $e^{-i t H}$ is a weak integral operator with jointly continuous integral kernel.

The solution of this would be a special case of the following.

Conjecture. Let $V_{-} \in K_{\nu}, V_{+} \in K_{\nu}^{\text {loc }}$ with $\left(1+x^{2}\right)^{-1} V_{+}$in the closure of $C_{0}^{\infty}$ in $K_{\nu}$-norm. Then for all $t>0, e^{-i t H}$ is a weak integral operator with jointly continuous integral kernel.

B.9. Trace ideal properties. Here we discuss when operators of the form $f(H) g(x)$ lie in trace ideals $G_{p}$. Such results are often of technical use. For definition and properties of $g_{p}$ including discussion of the case $H=-\Delta$, see [195]. Previous results for $f(H)=e^{-t H}$ appear in [194]. Here we extend these results to $f(H)=(H-z)^{-\alpha}$ for suitable $\alpha$. We recall the definition of the Birman-Solomjak spaces.

Definition. $l^{p}\left(L^{2}\right)=\left\{G \mid \Sigma_{j \in Z^{\nu}}\left[\int_{\Delta_{j}}|G(x)|^{2} d^{\nu} x\right]^{p / 2}=\|f\|_{p ; 2}^{p}<\infty\right\}$ where $\Delta_{j}$ is the unit cube centered at $j$.

We will prove

TheOREM B.9.1. Let $V_{-} \in K_{\nu}, V_{+} \in K_{\nu}^{\text {loc }}$. Let $f$ be a bounded Borel function on $\operatorname{spec}(H)$ obeying

$$
|f(x)| \leqslant C(1+|x|)^{-\alpha}
$$

for some $\alpha>v / 4$. Let $g \in L^{2}\left(R^{\nu}\right)$. Then $f(H) g(x)$ is in $\mathscr{G}_{2}$ (the Hilbert-Schmidt operators). 
THEOREM B.9.2. Let $V_{-} \in K_{\nu}, V_{+} \in K_{\nu}^{\text {loc }}$. Let $f$ be a bounded Borel function on $\operatorname{spec}(H)$ obeying (B28) for some $\alpha>\nu / 2$. Let $g \in l^{1}\left(L^{2}\left(R^{\nu}\right)\right)$. Then $f(H) g(x)$ is in $G_{1}$ (the trace class).

REMARKS 1. One might hope that (B28) could be replaced by some kind of falloff of a local $L^{2}$-norm of $f$ but the existence of point spectrum (even dense point spectrum) shows that $f$ bounded is absolutely essential.

2. The above theorems and interpolation immediately imply

TheOREM B.9.3. Let $V_{-} \in K_{\nu}, V_{+} \in K_{\nu}^{\text {loc }}$. Let $f$ be a bounded Borel function on $\operatorname{spec}(H)$ obeying (B28) for some $\alpha$. If $p \geqslant 2$ and $\alpha>\nu / 2 p$, then $f(H) g(x)$ is in $\mathscr{G}_{p}$ whenever $g \in L^{p}\left(R^{\nu}\right)$. If $1 \leqslant p \leqslant 2$, and $\alpha>\nu / 2 p$, the $f(h) g(x)$ is in $\Phi_{p}$ whenever $g \in l^{p}\left(L^{2}\right)$.

Theorem B.9.1 follows immediately from Theorem B.2.1 and

Proposition B.9.4. Let $A$ be a bounded operator on $L^{2}\left(R^{\nu}\right)$ so that $A^{*}$ maps $L^{2}$ to $L^{\infty}$. Then $A g(x)$ is in $\oiint_{2}$ for any $g \in L^{2}$.

Proof. By Corollary A.1.2, $A^{*}$ is an integral operator with kernel $A^{*}(x, y)$ obeying $\sup _{x} \int\left|A^{*}(x, y)\right|^{2} d y<\infty$. Thus $\bar{g} A^{*}$ is an integral operator with kernel in $L^{2}$ so $\bar{g} A^{*}$ is in $\Phi_{2}$. Thus $\left(\bar{g} A^{*}\right)^{*}=A g$ is in $\Phi_{2}$.

The proof of Theorem B.9.2 begins with a similar result.

Proposition B.9.5. Let $A, B$ be bounded operators on $L^{2}\left(R^{\nu}\right)$ so that $B$ is bounded from $L^{2}$ to $L^{\infty}$ and $A$ is bounded from $L_{-\delta}^{2}$ to $L_{-\delta}^{\infty}$ for some $\delta>\nu / 2$. Then for any $g \in L_{\delta}^{2}, g A B$ is trace class with

$$
\|g A B\|_{1} \leqslant C\|g\|_{2, \delta}\|A\|_{2,-\delta ; \infty,-\delta}\|B\|_{2, \infty} .
$$

Proof. Write $g A B=C D$ with $C=g\left(1+x^{2}\right)^{\delta / 2}\left[\left(1+x^{2}\right)^{-\delta / 2} A\left(1+x^{2}\right)^{\delta / 2}\right]$ and $D=\left(1+x^{2}\right)^{-\delta / 2} B$. By the last proposition, $C$ and $D$ are Hilbert-Schmidt.

Proof of Theorem B.9.2. Fix $\delta>0$. By Theorems B.2.1, B.6.3 and the last proposition $\left\|g(H-z)^{-\alpha}\right\|_{1} \leqslant C\|g\|_{2, \delta}$ with a constant $C$ depending only on the $K_{\nu}$ norm of $V_{-}$. Since this is translation invariant, we see that

$$
\left\|g(H-z)^{-\alpha}\right\|_{1} \leqslant C\left\|\left(1+|x-j|^{2}\right)^{\delta / 2} g\right\|_{2} .
$$

Thus, for any $g \in l^{1}\left(L^{2}\right)$

$$
\begin{aligned}
\left\|g(H-z)^{-\alpha}\right\|_{1} & \leqslant \sum_{j}\left\|g \mathscr{X}_{j}(H-z)^{-\alpha}\right\|_{1} \\
& \leqslant C \sum_{j}\left\|g\left(1+|x-j|^{2}\right)^{\delta / 2} \mathscr{X}_{j}\right\|_{2} \leqslant C^{\prime} \sum_{j}\left\|g \mathfrak{X}_{j}\right\|_{2}
\end{aligned}
$$

where $\mathscr{X}_{j}$ is the characteristic function of $\Delta_{j}$.

In [44], Cwickel proved that if $2<p<\alpha$ and $f, g$ lie in the weak $L^{p}$-space $L_{w}^{p}\left(R^{\nu}\right)$, then $f(i \nabla) g(x)$ lies in the weak trace ideal [195], $\Phi_{w}^{p}$. This leads to

Open problem. Fix $V_{+} \in K_{v}^{\text {loc }}, V_{-} \in K_{v}$ and $2<p<\infty$. Is it true that for every $g$ in $L_{w}^{p}$ and $\alpha=\nu / 2 p$, the operator $(H-z)^{-\alpha} g(x)$ lies in $G_{w}^{p}$ ?

This question is primarily of academic interest, in that it does not have the immediate interest of Cwickel's results. 
There is a final result on trace class properties due (in a slightly weaker form) to Davies [47] for which we give a new proof.

TheOREM B.9.6 (DAVIES [47]). Let $V \geqslant 0, V \in K_{\nu}^{\text {loc }}$. Let $\mathfrak{X}$ be the characteristic function of a bounded set, $K$. Then $\mathcal{X} V^{1 / 2}(H+1)^{-\alpha}$ is in the Hilbert-Schmidt class so long as $\alpha>\frac{1}{2}+\frac{1}{4} \nu$ with Hilbert-Schmidt norm bounded by a constant $C$ depending only on $K$ and not on $V$.

REMARK. The interest depends on the independence of $V$ so that one can take limits to non- $K_{\nu} V$, even non- $L^{1}-V$. Since $f(x)\left(H_{0}+1\right)^{-\alpha}$ is in $g_{2}$ if and only if $f \in L^{2}$ [195], the fact that non- $L^{1} V$ are allowed is especially interesting. Of course the $\infty$ in $\int\left|V^{1 / 2}(x)\right|^{2}$ is somehow cancelled by a smallness in $\left(H_{0}+V+1\right)^{-\alpha}$; indeed the extra 1 in the condition $2 \alpha>\nu / 2+1$ (rather that $\alpha>\nu / 4)$ is due to the need for cancellation.

Proof. We prove that the diagonal of the formal integral kernel of $(H+1)^{-\alpha} \mathcal{X V}(H+1)^{-\alpha}$ is integrable using the formal path integral formulae [194]. It is easy to justify everything. We first use (A9) to write

$$
\begin{aligned}
\operatorname{Tr}\left((H+1)^{-\alpha} \mathscr{X}(H+1)^{-\alpha}\right) & =c \int_{0}^{\infty} d t d s t^{\alpha-1} s^{\alpha-1} e^{-t-s} \operatorname{Tr}\left(e^{-t H} X V e^{-s H}\right) \\
& \leqslant c \int_{0}^{\infty} d w w^{2 \alpha-2} e^{-w} \int_{0}^{w} d t \operatorname{Tr}\left(e^{-t H} X V e^{-(w-t) H}\right) .
\end{aligned}
$$

Let $E_{x, x ; w}$ denote expectation with respect to Brownian motion starting at $x$ and conditioned to end at $x$ at time $w$. Then

$$
\begin{gathered}
(\mathrm{B} 29) \leqslant c \int d^{\nu} x \int_{0}^{\infty} d w w^{2 \alpha-2} e^{-w} \int_{0}^{w} d t(2 \pi w)^{-\nu / 2} \\
E_{x, x ; w}\left(e^{-\int_{0}^{w} V(b(s)) d s} \mathfrak{X}(b(t)) V(b(t))\right) .
\end{gathered}
$$

Let $\tilde{X}_{x, w}(b)$ the characteristic function of all paths with $b(s) \in K$ for some $s \leqslant w$ and $g(y)=y e^{-y}$. Then

$$
\begin{aligned}
(\mathrm{B} 30) & \leqslant c^{\prime} \int d^{\nu} x \times \int_{0}^{\infty} d w w^{2 \alpha-2-\nu / 2} e^{-w} E_{x, x ; w}(\tilde{X}(b)) g\left(\int_{0}^{t} V(b(s)) d s\right) \\
& \leqslant c^{\prime \prime} \int d^{\nu} x \times \int_{0}^{\infty} d w w^{2 \alpha-2-\nu / 2} e^{-w} E_{x, x ; w}(\tilde{X}(b)) \\
& \leqslant c^{\prime \prime \prime} \int d^{\nu} x \times \int_{0}^{\infty} d w w^{2 \alpha-2-\nu / 2} e^{-w} \exp \left(-d w^{-1} \operatorname{dist}(x, K)^{2}\right) \leqslant \tilde{C} .
\end{aligned}
$$

The $c^{\prime \prime}$ estimate follows from $\sup _{y \geqslant 0} g(y)=e^{-1}$ and the $c^{\prime \prime \prime}$ by an elementary estimation of paths ( $c^{\prime \prime \prime}$ is $K$-dependent). Since $2 \alpha-2-\frac{1}{2} \nu>-1$, the last estimate follows from the finiteness of the integral.

B10. Continuity in $V$. Continuity of the map $V \mapsto e^{-t\left(H_{0}+V\right)}$ as a map from $V$ 's to the bounded operator from $L^{p}$ to $L^{q}$ is a natural question. Moreover, in $\S \S \mathrm{B} 3$ and B7 we have seen that for proving continuity of $e^{-t H} f$ or of integral 
kernels it is useful to have such results. In this section we prove

Theorem B.10.1. Let $V, V_{m} \in K_{\nu}$ and let $\left\|V-V_{m}\right\|_{K} \rightarrow 0$. Then for any fixed $t, p, q$ with $t \geqslant 0, p \leqslant q$ we have that $\left\|e^{-t H_{m}}-e^{-t H^{\nu}}\right\|_{p, q} \rightarrow 0$ as $m \rightarrow \infty$.

THEOREM B.10.2. Let $V_{-}, V_{-, m} \in K_{\nu} ; V_{+}, V_{+, m} \in K_{\nu}^{\text {loc }}$. Suppose that

(i) $\lim _{t \downarrow 0} \sup _{m, x} E_{x}\left(\int_{0}^{t} V_{-, m}(b(s)) d s\right)=0$.

(ii) For any compact $K,\left\|\mathfrak{X}_{K}\left(V_{m}-V\right)\right\|_{K_{\nu}} \rightarrow 0$ where $\mathcal{X}_{K}$ is the characteristic function of $K$. Then

(a) for any $t>0$, any compact $K$ and any $p,\left\|\chi_{K}\left(e^{-t H}-e^{-t H_{m}}\right)\right\|_{p, p} \rightarrow 0$ and $\left\|\left(e^{-t H}-e^{-t H_{m}}\right) \mathcal{X}_{K}\right\|_{p, p} \rightarrow 0$.

(b) For any $t>0$, any compact $K$ and all $p \leqslant q$

$$
\left\|\mathfrak{X}_{K}\left(e^{-t H}-e^{-t H_{m}}\right) \mathfrak{X}_{K}\right\|_{p, q} \rightarrow 0 .
$$

REMARK. Given any $V$ with $V_{+} \in K_{\nu}^{\text {loc }}, V_{-} \in K_{\nu}$ it is easy to find $V_{m} \in C_{0}^{\infty}$ obeying hypotheses (i), (ii) of Theorem B.10.2, e.g. multiply $V$ by the characteristic function of a large set and then convolute with a spherically symmetric approximate delta function.

Proof of Theorem B.10.1. We first claim that it is no loss to suppose that $p=q$ for we can then handle the general $p \leqslant q$ by using Theorem B.1.1 and (B31)

$$
\begin{gathered}
\left\|e^{-2 t H}-e^{-2 t H_{m}}\right\|_{p, q} \leqslant\left\|e^{-t H}\left(e^{-t H}-e^{-t H_{m}}\right)\right\|_{p, q}+\left\|\left(e^{-t H}-e^{-t H_{m}}\right) e^{-t H_{m}}\right\|_{p, q} \\
\leqslant\left\|e^{-t H}\right\|_{p, q}\left\|e^{-t H}-e^{-t H_{m}}\right\|_{p, p}+\left\|e^{-t H}-e^{-t H_{m}}\right\|_{q, q}\left\|e^{-t H_{m}}\right\|_{p, q} .
\end{gathered}
$$

By duality and interpolation, it suffices to treat the case $p=q=\infty$. By using (B31) again it suffices to also prove the result only for $t \leqslant T$ for some small $T$. We can pick $T$ so small that the expansion whose terms are (B3) converges for each $V_{m}$ uniformly in $m$. It then suffices to show that for each $n$

$$
\sup _{t} E_{x}\left(\int_{0<s_{1}<\cdots<s_{n}<t}\left[\prod_{1}^{n} V_{m}\left(b\left(s_{1}\right)\right)-\prod_{1}^{n} V\left(b\left(s_{1}\right)\right)\right] d s_{1} \cdots d s_{n}\right) \rightarrow 0 .
$$

This follows by writing

$$
\left|\prod_{1}^{n} a_{i}-\prod_{1}^{n} b_{i}\right| \leqslant \sum_{j}\left[\prod_{1}^{j-1}\left|a_{j}\right| \prod_{j+1}^{n}\left|b_{j}\right|\right]\left[\left|b_{j}-a_{j}\right|\right]
$$

and using Lemma B.1.3.

Proof of Theorem B.10.2. Since it is somewhat lengthy, we break it into steps. As a notational preliminary, we denote by $K_{r}$ the set $\{x \mid \operatorname{dist}(x, K)<r\}$. We also note a fact from the theory of Brownian motion [194]: For each fixed $x$ and $t$

$$
\lim _{r \rightarrow \infty} P_{0}(|b(s)| \leqslant r, \text { all } 0 \leqslant s \leqslant t)=1 .
$$

This follows from the continuity a.e. of Brownian paths; in fact very good estimates on the probability are available; see [194].

Step 1. $\sup _{m}\left\|e^{-t\left(H_{0}+\alpha V_{m}\right)}\right\|_{p, q}<\infty ; p, q, \alpha>0, t>0$ fixed. This follows from hypothesis (i) and the proof of Theorem B.1.1. 
Step 2. $p=q=\infty ; \mathfrak{X}_{K}\left(e^{-t H}-e^{-t H_{m}}\right)$. We must show that

$$
\begin{aligned}
\sup _{\|f\|_{\infty} \leqslant 1} \sup _{x \in K} \mid E_{x}([ & \exp \left(-\int_{0}^{t} V(b(s)) d s\right) \\
& \left.\left.-\exp \left(-\int_{0}^{t} V_{m}(b(s)) d s\right)\right] f(b(t))\right) \mid \rightarrow 0 .
\end{aligned}
$$

Let $G_{m}$ be the integrand inside $E_{x}$, and let $\tilde{X}_{r}(b)$ be the characteristic function of the set of paths with $b(s) \in K_{r}$ for $0 \leqslant s \leqslant t$. Then, (B32) implies that

$$
\lim _{r \rightarrow \infty} \sup _{x \in K} E_{x}\left(1-\tilde{X}_{r}\right)=0 \text {. }
$$

By the Schwarz inequality

$E_{x}\left(G_{m}\left(1-\tilde{X}_{r}\right)\right) \leqslant\|f\|_{\infty} E_{x}\left(1-\tilde{X}_{r}\right)^{1 / 2}\left[\left\|e^{-t\left(H_{0}+2 V\right)} 1\right\|_{\infty}+\left\|e^{-t\left(H_{0}+2 V_{m}\right)} 1\right\|_{\infty}\right]$

go to zero uniformly in $x \in K$ and $\|f\|_{\infty} \leqslant 1$ as $r \rightarrow \infty$ by Step 1 and (B33). Now let $V_{m}^{(r)}, V^{(r)}$ be $V_{m}, V$ multiplied by $\mathscr{X}_{K_{r}}$ and let $G_{m}^{(r)}$ be the same as $G_{m}$ with $V_{m}, V$ replaced by $V_{m}^{(r)}, V^{(r)}$. As above

$$
\lim _{r \rightarrow \infty} \sup _{x \in K} E_{x}\left(G_{m}^{(r)}\left(1-\tilde{X}_{r}\right)\right)=0
$$

uniformly in $\|f\|_{\infty}$. Noting that $\left(G_{m}^{(r)}-G_{m}\right) \tilde{X}_{r}=0$, we see that it suffices that $\sup _{\|f\|_{\infty}<1} \sup _{x \in K} E_{x}\left(G_{m}^{(r)}\right) \rightarrow 0$ for each fixed $r$. But, by hypothesis (ii), this follows from Theorem B.10.1.

Step 3. $\lim _{r \rightarrow \infty} \sup _{m}\left\|\left(1-\mathscr{X}_{K_{r}}\right) e^{-t H_{m}} \mathfrak{X}_{K}\right\|_{\infty, \infty}=0$. By Step 2 and Lemma B.4.1, it suffices to prove it for $H_{m}$ replaced by $H_{0}$, which follows from the explicit integral kernel.

Step 4. $p=q=\infty ;\left(e^{-t H}-e^{-t H_{m}}\right) \mathcal{X}_{K}$. By Step 3, given $\varepsilon$, we find $r$ so that

$$
\sup _{m}\left\|\left(1-\mathcal{X}_{K_{r}}\right)\left[e^{-t H}-e^{-t H_{m}}\right] \mathcal{X}_{K}\right\|_{\infty, \infty}<\varepsilon .
$$

By Step 2, $\lim _{m \rightarrow \infty}\left\|\mathscr{X}_{K_{r}}\left[e^{-t H}-e^{-t H_{m}}\right] \mathscr{X}_{K}\right\|_{\infty, \infty} \rightarrow 0$.

Step 5 . General $p=q$. Follows from Steps 2 and 4 by duality and interpolation.

Step 6. General $p, q$. Follows from the obvious generalization of (B31).

B11. Hypercontractive semigroups and all that. In this section, we will summarize some of the literature associated with the terms "hypercontractive" and "supercontractive". Given a normalized function $\Omega$ in $L^{2}\left(R^{\nu}, d^{\nu} x\right)$ with $\Omega>0$, let $d \mu$ be the probability measure

$$
d \mu(x)=\Omega(x)^{2} d x .
$$

There is a natural unitary map $U_{\Omega}: L^{2}\left(R^{\nu}, d^{\nu} x\right) \rightarrow L^{2}\left(R^{\nu}, d \mu(x)\right)$ by $\left(U_{\Omega} f\right)(x)$ $=\Omega(x)^{-1} f(x)$. Any Schrödinger operator, $H$, carries over to an operator $H_{\Omega}$ on $L^{2}\left(R^{\nu}, d \mu\right)$ by $H_{\Omega}=U_{\Omega} H U_{\Omega}^{-1}$. Notice that

$$
e^{-t H_{\Omega}}=\Omega^{-1} e^{-t H} \Omega \text {. }
$$

What will concern us is whether $e^{-t H_{\Omega}}$ is bounded from $L^{p}\left(R^{\nu}, d \mu\right)$ to $L^{q}\left(R^{\nu}, d \mu\right)$. This is seen to be equivalent to asking if

$$
\Omega^{-1+2 q^{-1}} e^{-t H} \Omega^{1-2 p^{-1}}
$$


is bounded from $L^{p}\left(R^{\nu}, d^{\nu} x\right)$ to $L^{q}\left(R^{\nu}, d^{\nu} x\right)$. Thus, except for the case $p=q$ this question is related to but quite distinct from the question of bounded $e^{-t H}$ from a weighted $L^{p}$ to a weighted $L^{q}$. Indeed, since we will generally be interested in the case $p<q$ (since $q \leqslant p$ will be so easy for the "natural" choice of $\Omega$ ), and $\Omega^{2 q^{-1}-2 p^{-1}}$ will then grow at infinity, there is no real hope that $e^{-t H_{a}}$ will go from $L^{p}$ to $L^{q}$ unless $V$ grows at infinity so that there is some falloff of $e^{-t H}$ as $x$ and $y$ go to infinity (even with $|x-y| \rightarrow 0$ ). Since we already know about local smoothing of $e^{-t H}$ it is clear that the question being asked here is exactly about behavior at infinity. After we make the choice of $\Omega$, we will examine this notion further.

There is one especially natural choice of $\Omega$. Suppose that $H$ has an $L^{2}$-eigenfunction $\Omega>0$ so that $H \Omega=E \Omega$ (of necessity, since $\Omega>0, E=\inf \operatorname{spec}_{L^{2}}(H)$; see [162, §XIII.12]). If we take this choice of $\Omega$, then $e^{-t\left(H_{\mathrm{R}}-E\right)} 1=1$ and we have

Proposition B.11.1 [196]. If $e^{-t A}$ is a semigroup on $L^{2}(\Omega, d \nu)$ with $\nu$ a probability measure and if (i) $e^{-t A} f \geqslant 0$ then $f \geqslant 0$, (ii) $e^{-t A} 1=1$, then $e^{-t A}$ is a contraction from any $L^{p}$ to any $L^{q}$ with $q \leqslant p$.

Remark. Since $L^{q} \supset L^{p}$ for $q \leqslant p$, the only interesting case is $q=p$. For a proof see [196] or [161, §X.8].

Henceforth, we always pick this $\Omega$ and change $V$ by a constant so that $H \Omega=0$. With this choice, one formally has that

$$
\int \overline{f(x)}\left(H_{\Omega} g\right)(x) \Omega^{2} d x=\int \overline{\nabla f(x)} \cdot \nabla g(x) \Omega^{2} d x
$$

something that can be proven for a core of $H$ in many concrete situations (see e.g., $[78,163,10])$. Because of (B36), the operators $H_{\Omega}$ are often called Dirichlet forms.

If $\Omega=e^{-h}$, then $V=\frac{1}{2}\left((\nabla h)^{2}-\Delta h\right)$. If $h$ goes to infinity in a regular way, $\Delta h$ is smaller than $(\nabla h)^{2}$ so one sees that if $V \sim x^{a}$, then $h \sim x^{1+a / 2}$. In particular, $\Omega^{2 q^{-1}-2 p^{-1}} e^{-t V}$ will be unbounded at infinity if $a<2$, bounded at infinity for all $t>0, p, q$ if $a>2$ and bounded at infinity for $p, q$ depending on $t$ if $a=2$. This suggests qualitatively the precise behavior in the two theorems below (Theorems B.11.3 and B.11.4) although the quantitative prediction one would make when $a=2$ about $p, q$ is wrong.

Definition. A semigroup $e^{-t A}$ on $L^{2}(\Omega, d \nu)$ with $\Omega$ a probability measure space is called hypercontractive if and only if it is a contraction on each $L^{p}(\Omega, d \nu)$ and for some $t, e^{-t H}$ is bounded from $L^{2}$ to $L^{4}$. Carmona [34] has made this intuition precise.

General principles imply that there is then $T(p, q)$ with $e^{-t H}$ bounded from $L^{p}$ to $L^{q}$ if $t>T(p, q)$. Because of Theorem B.11.3 below (see the comments following it), hypercontractive semigroups played an important role in the mathematical development of quantum field theory in the period 1965-1971, especially in the germinal papers of Nelson [141], Glimm [73] and Glimm and Jaffe $[74,75]$. As a result, an abstract theory was developed originally by Segal [175] with further developments in $[55,56,85,176,178,196]$. A typical result is the following abstracted from Glimm [73]. 
Proposition B.11.2. If $e^{-t H}$ is bounded from $L^{2}$ to $L^{4}$, and if $H 1=0$, $H \uparrow\{1\}^{\perp} \geqslant m>0$, then $e^{-t H}$ is a contraction from $L^{2}$ to $L^{4}$ for $t$ sufficiently large.

A central example in the development is

THEOREM B.11.3 ("NELSON'S BEST HYPERCONTRACTIVE ESTIMATES"). Let $V(x)=\frac{1}{2} x^{2}-\frac{1}{2} \nu$. Then $e^{-t H_{\Omega}}$ is bounded from $L^{p}$ to $L^{q}$ if and only if

$$
e^{-2 t} \leqslant(p-1) /(q-1)
$$

and in that case it is a contraction.

REMARK. By a scaling argument, one obtains the same result if

$$
V(x)=\frac{1}{2} \sum_{i=1}^{\nu}\left(\omega_{i}^{2} x_{2}^{2}-\omega_{i}\right)
$$

so long as $\min \omega_{i}=1$. In quantum field theory, one deals essentially with the $\nu=\infty$ limit of such operators in describing free frields and the estimates carry through and are useful in treating perturbations of the free field.

Hypercontractivity of the above semigroup was first proven by Nelson [141] for any finite $\nu$. Glimm [73] proved Proposition B.11.2 in the concrete setting precisely to show that $e^{-t H}$ is a contraction which allows a $\nu$-independent bound critical for the field theory. The "best estimates" were then proven by Nelson [142]. They have evoked a considerable literature, much of it in terms of some inequalities (called "logarithmic Sobolev inequalities") which Gross [78] showed were equivalent to Theorem B.11.3:

$$
\int|\Psi|^{2} \ln |\Psi| d \mu \leqslant\langle\Psi, H \Psi\rangle+\|\Psi\|_{2}^{2} \ln \|\Psi\|_{2}
$$

and for which Gross provided an intriguing proof. Besides the proofs of Gross [78] and Nelson [142] of Theorem B.11.3, we mention that of Brascamp and Lieb [29] and for the case $p=2, q=4$ of Simon [183]. See Rothhaus [164-167] and Weissler [205] for additional literature.

Definition. A semigroup $e^{-t A}$ on $L^{2}$ of a probability measure space is called supercontractive if and only if $e^{-t A}$ maps $L^{p}$ to $L^{q}$ for any $1<p<q<\infty$ and all $t>0$.

THEOREM B.11.4 (ROSEN [163] FOR $\alpha>2$; CARMONA [34] FOR $\alpha<2$ ). Let $V$ obey

$$
C_{1}\left(1+|x|^{2}\right)^{\alpha / 2}-C_{2} \leqslant V(x) \leqslant C_{3}\left(1+|x|^{2}\right)^{\alpha / 2}
$$

for some $\alpha>0, C_{1}>0$. Let $\Omega>0$ with $H \Omega=0$. Then $e^{-t H_{\Omega}}$ is supercontractive if $\alpha>2$ and not bounded from any $L^{p}$ to any $L^{q}$ with $p<q$ for any $t>0$ if $\alpha<2$.

See Rosen [163] for the case $\alpha>2, \nu=1$ and Simon [182] for how to extend to higher $\nu$. Prior to Rosen's work, Eckmann [52] proved the $\nu=1, \alpha>2$ groups were hypercontractive. Both Eckmann and Rosen use ideas of Gross [78]. Carmona [34] proved the $\alpha<2$ implicitly given lower bounds on the decay of $\Omega[182,35,37]$. See Hooton $[96,97]$ and Carmona [34] for additional discussion and results on $e^{-t H_{\Omega}}$. 
Open question. If (B38) holds with $\alpha<2$, is it true that $e^{-t H_{\Omega}}$ is not bounded from $L^{p}$ to $L^{q}$ for any $t>0$ and $p<q$ ?

B12. Some remarks on the case when $H$ is unbounded below. The methods of Faris and Lavine [58] or Kato [112] imply

THEOREM B.12.1. Let $H=-\frac{1}{2} \Delta+V+W$ where $V_{+} \in K_{\nu}^{\text {loc }}, V_{-} \in K_{\nu}, V \in$ $L_{\text {loc }}^{2}$ and

$$
|W(x)| \leqslant C\left(1+x^{2}\right)^{\mu / 2}
$$

for some $\mu \leqslant 2$. Then $H$ is essentially selfadjoint on $C_{0}^{\infty}\left(R^{\nu}\right)$.

For such $H$ 's, it can happen that inf $\operatorname{spec}(H)=-\infty$ so $e^{-t H}$ is not bounded on $L^{2}$. Thus such operators cannot be directly studied by the methods in this paper. However, one can learn about $H$ by using properties of $\tilde{H}=\frac{1}{2} \Delta+V$. We want to illustrate this by proving the following result which is important because it implies that such $H$ 's have continuum eigenfunction expansions with polynomially bounded eigenfunctions; see §C5. While the restriction from $\mu \leqslant 2$ to $\mu \leqslant 1$ is unfortunate, the important case of atoms in constant electric field $[17,80,82]$ is included; this result is new. Some of the techniques come from $[17,186]$.

THEOREM B.12.2. Let $H$ have the form of Theorem B.12.1 with $\mu \leqslant 1$. Fix an integer $k$. Then $\left(1+x^{2}\right)^{-k}(H-i)^{-k}$ is in the trace ideal $9_{p}$ for any $p>v / k$. In particular, the operator is trace class for $k=\nu+1$.

LEMMA B.12.3. Under the hypothesis of Theorem B.12.1,

$$
\left(1+x^{2}\right)^{-\mu / 2} p(H-z)^{-1} \text { and } p\left(1+x^{2}\right)^{-\mu / 2}(H-z)^{-1}
$$

are bounded with norms going to zero as $\kappa \rightarrow-\infty$ if $z=\kappa+i$. The same is true of $\left(1+x^{2}\right)^{-\mu / 2}(H-z)^{-1}$.

Proof. Let $\tilde{H}=H_{0}+V$ and use

$$
\begin{aligned}
\left(1+x^{2}\right)^{-\mu / 2} p(H-z)^{-1}= & \left(1+x^{2}\right)^{-\mu / 2} p(\tilde{H}-z)^{-1} \\
& -\left(1+x^{2}\right)^{-\mu / 2} p(\tilde{H}-z)^{-1} W(H-z)^{-1}
\end{aligned}
$$

and Theorem B.6.5. The last statement has a similar proof.

Proposition B.12.4. For some $z,\left(1+x^{2}\right)^{-\alpha / 2}(H-z)^{-1}\left(1+x^{2}\right)^{\alpha / 2}$ is bounded for all $\alpha$ with $|\alpha| \leqslant \nu+1$.

Proof. Let $\rho$ be multiplication by $\left(1+x^{2}\right)^{1 / 2}$ and let $\varphi=\rho^{-1} \nabla \rho$ so $|\varphi| \leqslant$ $\rho^{-1}$. For $\alpha$ purely imaginary

$$
\rho^{\alpha} H \rho^{-\alpha}=H-\alpha^{2} \varphi^{2}+\alpha \varphi \nabla+\alpha(\nabla) \varphi \equiv H+F(\alpha) .
$$

Notice $F$ is defined for all complex $\alpha$. By the lemma, since $\mu \leqslant 1$,

$$
\lim _{\substack{z \rightarrow \infty \\ z=x+i}}\left\|F(\alpha)(H-z)^{-1}\right\|=0 .
$$

By standard perturbation theory [114], it follows that $(H+F(\alpha)-z)$ is invertible for all $|\alpha| \leqslant \nu+1$ if $z=x+i$ with $x$ near $-\infty$. But for $\alpha$ purely 
imaginary $(H+F(\alpha)-z)^{-1}=\rho^{\alpha}(H-z)^{-1} \rho^{-\alpha}$. It follows (see e.g. [43]) that $\rho^{\alpha}(H-z)^{-1} \rho^{-\alpha}$ is bounded for all $|\alpha| \leqslant \nu+1$.

Proposition B.12.5. For suitable $z$ and all $\alpha$ with $|\alpha| \leqslant \nu+1$,

$$
p\left(1+x^{2}\right)^{-(\alpha+\mu / 2)}(H-z)^{-1}\left(1+x^{2}\right)^{\alpha / 2}
$$

is bounded.

Proof. Follow the proof of Lemma B.12.3, using Proposition B.12.4 to control the new terms $\left(1+x^{2}\right)^{-\alpha / 2}(H-z)^{-1}\left(1+x^{2}\right)^{\alpha / 2}$ which replace $(H-z)^{-1}$.

Proposition B.12.6. For suitable $z$ and all $|\alpha| \leqslant \nu+1$

$$
\left(1+x^{2}\right)^{-(\alpha+\mu+1) / 2}(H-z)^{-1}\left(1+x^{2}\right)^{\alpha / 2}
$$

lies in the trace ideal $\Phi_{q}$ for all $q>\nu$.

Proof. By the last two propositions

$$
(1+|p|)\left(1+x^{2}\right)^{-(\alpha+\mu) / 2}(H-z)^{-1}\left(1+x^{2}\right)^{\alpha / 2}
$$

is bounded. Since $\left(1+x^{2}\right)^{-1 / 2}(1+|p|)^{-1}$ is in all $\Phi_{q}, q>\nu$ (see e.g., $\$ 4$ of [195]), we are done.

Proof OF THEOREM B.11.2. Write

$$
\left(1+x^{2}\right)^{-k}(H-z)^{-k}=A_{k} A_{k-1} \cdots A,
$$

with $A_{j}=\left(1+x^{2}\right)^{-j}(H-z)^{-1}\left(1+x^{2}\right)^{j-1}$ and use the proposition together with Hölder's inequality for trace ideals [195].

B13. The magnetic case. We want to explain here why all $L^{p}$-estimates we have discussed for resolvents and semigroups hold if $H$ is replaced by

$$
H(a) \equiv H_{0}(a)+V ; \quad H_{0}(a)=\frac{1}{2}(-i \nabla-a)^{2} .
$$

The following is basic to the study of such operators.

Proposition B.13.1 (SimON [187]). Let $\vec{a} \in L_{\mathrm{loc}}^{2}$ and let $H_{0}(a)$ denote the selfadjoint operator whose form domain is $\left\{f \in L^{2} \mid(i \vec{\nabla}+\vec{a}) f \in L^{2}\left(R^{\nu} ; R^{\nu}\right)\right\}$ with $\left(f, H_{0}(a) f\right)=\|(i \vec{\nabla}+\vec{a}) f\|^{2}$. Then for any $g$, we have pointwise that

$$
\left|e^{-t H_{0}(a)} g\right| \leqslant e^{-t H_{0}}|g| \text {. }
$$

REMARKS. 1. since $\vec{a} f \in L_{\mathrm{loc}}^{1}$ it defines a distribution and the symbol $i \vec{\nabla}+\vec{a} f$ $\in L^{2}$ means the distributional sum lies in $L^{2}$.

2. (B40) for smooth $a$ 's first appeared in a paper by Simon [184], who used a stochastic integral proof of Nelson. One subsequent proof $[84,185]$ related it to a basic distributional inequality of Kato [112]. Kato [113] first worried about removing the restrictions on $\vec{a}$ and raised the general $L_{\text {loc }}^{2}$ question which was answered in Simon [187].

3. The proof in [187] for smooth $\vec{a}$ (to avoid technical difficulties) is illuminating. For each $j$, one can find $\lambda_{j}$ so that $a_{j}=\partial_{j} \cdot \lambda_{j}$ (no summation of indices!). Then

$$
\left(i \partial_{j}+a_{j}\right)=e^{\lambda_{j}}\left(i \partial_{j}\right) e^{-\lambda_{j}}
$$


so

$$
\exp \left(-t\left(i \partial_{j}+a_{j}\right)^{2}\right)=e^{\lambda_{j}} \exp \left(t \partial_{j}^{2}\right) e^{-\lambda_{j}}
$$

(B40) now follows from the fact that $\exp \left(t \partial_{j}^{2}\right)|f| \geqslant 0$ and the Trotter product formulae:

$$
\begin{aligned}
\exp \left(-t H_{0}(a)\right) & =\operatorname{s-lim}_{n \rightarrow \infty}\left(\exp \left[-t\left(i \partial_{1}+a_{1}\right)^{2} / n\right] \cdots \exp \left[-t\left(i \partial_{\nu}+a_{\nu}\right) 2 / n\right]\right)^{n}, \\
\exp \left(-t H_{0}\right) & =s-\lim _{n \rightarrow \infty}\left(\exp \left(+t \partial_{1}^{2} / n\right) \cdots \exp \left(t \partial_{\nu}^{2} / n\right)\right)^{n} .
\end{aligned}
$$

One consequence of (B40) is that for any function $f$,

$$
\left\||f|^{1 / 2}\left(H_{0}(a)+c\right)^{-1}|f|^{1 / 2}\right\| \leqslant\left\||f|^{1 / 2}\left(H_{0}+c\right)^{-1}|f|^{1 / 2}\right\|
$$

since the integral of one operator is dominated by the other. Thus, if $V_{-} \in K_{\nu}$, $V_{-}$is $H_{0}(a)$ form bounded with relative bound zero and so $H_{0}(a)+V$ can be defined as a form with form domain $Q\left(H_{0}(a)\right) \cap Q\left(V_{+}\right)$. By mimicking the proof we gave of Theorem B.1.5, one proves [187]

TheOREM B.13.2. Let $a \in L_{\text {loc }}^{2}, V_{+} \in K_{\nu}^{\text {loc }}, V_{-} \in K_{\nu}$. Then $C_{0}^{\infty}$ is a form core for $H_{0}(a)+V$ and pointwise

$$
\left|\exp \left[-t\left(H_{0}(a)+V\right)\right] g\right| \leqslant \exp \left[-t\left(H_{0}+V\right)\right]|g| \text {. }
$$

(B41) immediately implies that

CorollaRy B.13.3. For any $V, p, q, t, \alpha$ and $z$ with $\operatorname{Re} z<\inf \operatorname{spec}(H)$

$$
\begin{gathered}
\left\|e^{-t H(a)}\right\|_{p, q} \leqslant\left\|e^{-t H}\right\|_{p, q}, \\
\left\|(H(a)-z)^{-\alpha}\right\|_{p, q} \leqslant\left\|(H-\operatorname{Re} z)^{-\alpha}\right\|_{p, q} .
\end{gathered}
$$

In particular, $e^{-t H(a)}$ maps $L^{p}$ to $L^{q}$ for all $t>0$ and $q \geqslant p$ and $(H(a)-z)^{-\alpha}$ maps $L^{p}$ to $L^{q}$ so long as (B12) holds.

Open question. Does $e^{-t H(a)}$ map $L^{\infty}$ to continuous function? To function with $L_{\text {loc }}^{2}$ gradients? Are the integral kernels continuous?

The proof of Theorem B.1.6 does not carry over but the analogous result has been proven by Leinfelder and Simader [129] using an ingenious argument (see Schechter [171, 172] and Simon [179, 187] for earlier results).

TheOREM B.13.4 (LeINFelder AND Simader [129]). If $a \in L_{\mathrm{loc}}^{4}, V \in L_{\mathrm{loc}}^{2}$, $V_{-} \in K_{\nu}$, then $C_{0}^{\infty}$ is an operator case for $H(a)$.

Gauge invariance of $H(a)$ is studied by Leinfelder in [128]. Among the results there is the following, which is of interest even if $a=0$.

TheOREM B.13.5 (LeINFELder [128]). Let $V_{+} \in K_{\nu}^{\text {loc }}, V_{-} \in K_{\nu}, a \in L_{\text {loc }}^{2}$. Then the essential spectrum of $H(a)$ is either empty or an unbounded set.

\section{EIGENFUnCTIONS}

C1. Harnack's inequality and subsolution estimates. In this chapter, we study properties of eigenfunctions of $H$ and the consequences of these properties for the spectrum of $H$ and for the various functions, $f(H)$, studied in Chapter B. 
For early results on eigenfunctions, we mention especially the germinal paper of Kato [110]. In this section, we discuss two intimately related aspects of bounds on eigenfunctions: (1) Harnack's inequality, i.e. a priori bounds on $u(x) / u(y)$ for functions $u$ obeying $H u=0, u \geqslant 0, u \neq 0$ on $\Omega \ni x, y$ with the bound depending only on $V, x, y, \Omega$ and not on $u$ and (2) subsolution estimates, i.e. bounds of the form

$$
|u(x)| \leqslant C \int_{|x-y| \leqslant 1}|u(y)| d^{\nu} y
$$

for $u$ 's obeying $H u=0$ in $\{y|| x-y \mid \leqslant 2\}$ where $C$ depends only on local norms of $V$. In discussing both, we consider only solutions of $H u=0$ but since $V$ can be replaced by $V-E$, we will have results automatically also for solutions of $H u=E u$. The constants will be $E$ dependent.

(C1) is obviously related to the theme of $\S \mathrm{B} 1$ : If $u$ obeys $H u=0$ globally and $u \in L^{1}$, then

$$
|u(x)| \leqslant\|u\|_{\infty} \leqslant C \int|u(y)| d^{\nu} y
$$

with $C=\left\|e^{-H}\right\|_{1, \infty}$. Thus (C1) is a kind of "local version" of the bounds of $\S B 1$. In fact, as we shall explain, its probabilistic proof is very close to that used in $\S \mathrm{B} 1$.

There are two rather distinct approaches to proving the kinds of estimates we present here. An analytic method of Trudinger [203] (see also [72]) relies on ideas of Moser [137] and Stampacchia [199]. Although Trudinger's hypotheses are slightly stronger than $V \in K_{\nu}^{\text {loc }}$, recently Agmon [3] has used an analytical approach to obtain Harnack's inequality for $V \in K_{\nu}^{\text {loc }}$. Moreover, large parts of the theory of eigenfunctions, especially if one settles for strengthened hypotheses on $V$ follow from clever uses of subharmonic comparison theorems; see Simon [182], M. and/or T. Hoffman-Ostenhof [86, 90-95, 8] and Davies [48].

The other approach is probabilistic. In the context of a general class of Markov processes but with $V \leqslant 0$, it goes back to Khasmin'skii [115]; indeed Lemma B.1.2 was developed in connection with this work. More recently, Chung and Varadhan [38], Chung and Rao [39], and Aizenman and Simon [9] have developed this approach. The proof in the Chung and Rao [39] is quite elegant but requires $V$ to be (locally) bounded. Aizenman and Simon [9] have the $K_{\nu}^{\text {loc }}$ result independently of Agmon. They also show that if $V \leqslant 0$, then a strong version of Harnack's inequality implies that $V \in K_{\nu}^{\text {loc }}$, showing once more the naturalness of the class. In this section, we state the main results in this vein and sketch parts of the proofs. For complete proofs, see [9].

As a preliminary, the following is useful.

TheOREM C.1.1 [9]. Let $V \in K_{\nu}^{\text {loc }}$ and let $H u=0$ in distributional sense on some bounded open set $\Omega \subset R^{\nu}$ in the sense that $u \in L_{\mathrm{loc}}^{1}, V u \in L_{\mathrm{loc}}^{1}$ and $\left(\frac{1}{2} \Delta \varphi, u\right)-(\varphi, V u)=0$ for all $\varphi \in C_{0}^{\infty}(\Omega)$. Then $u$ equals $($ a.e. $)$ a continuous function on $\Omega$. 
The proof [9] first shows, using the estimates of Chapter B, that $u \in L_{\text {loc }}^{\infty}$ and then one writes $u=f+g$ where $g=2(-\Delta)^{-1} V \mathcal{X} u$ with $\mathcal{X}$ the characteristic function of $\Omega$. By Proposition A.2.4(c), $g$ is continuous and $f$ can be seen to be harmonic on $\Omega$ and so continuous.

The first main result is

Theorem C.1.2 ([9, 203] Subsolution estimate). Let $V \in K_{\nu}^{\text {loc }}$ and let $H u=0$ on some open set $\Omega$. Pick $x \in \Omega$. Then for any $r>0$ with $\{x|| x-y \mid \leqslant$ $r\} \subseteq \Omega$ :

$$
|u(x)| \leqslant C \int_{|x-y| \leqslant r}|u(y)| d^{\nu} y
$$

where $C$ only depends on $r$ and the $K_{\nu}$ norm of $V_{-}$times the characteristic function of $\{y|| x-y \mid \leqslant r\}$, but not on $u$. In particular, if $V_{-} \in K_{\nu}$, and $\Omega=R^{\nu}$, then the constant $C$ in $\left(\mathrm{Cl}^{\prime}\right)$ can be chosen dependently of $x$.

Theorem C.1.2 follows immediately from an even stronger estimate:

Theorem C.1.3. Let $V \in K_{\nu}^{\text {loc }}$ and let $H u=0$ on some open set $\Omega$. Pick $x \in \Omega$. Then for some $r_{0}>0$,

$$
|u(x)| \leqslant C \int_{|x-y|=r}|u(y)| d \sigma(y)
$$

where do is the usual surface measure and, for any $r_{1}>0, C$ can be chosen independently of $r$ for $r_{1}<r<r_{0}$. The values of $C$ and $r_{0}$ depend only on local norms of $V_{-}$.

(C2) implies $\left(\mathrm{Cl}^{\prime}\right)$ by integrating (C2) over $r$ in $\left(r_{1}, r_{2}\right)$. The above estimate holds also for suitable regular $u$ 's obeying $u \geqslant 0, H u \leqslant 0$ [9]. The other main result is

TheOREM C.1.3 $\left([9,203]\right.$ HARNACK'S InEQUALITY). Let $V \in K_{\nu}^{\text {loc }}$. Let $\Omega$ be an open set with $x, y \in \Omega$. Then, there is a constant $C$ with

$$
u(x) \leqslant C u(y)
$$

for all continuous $u$ on $\Omega$ obeying (i) $u \geqslant 0$, (ii) $H u=0$ on $\Omega$. The constant $C$ can be chosen independently of $x, y$ as $x, y$ run through a compact subset $K$ of $\Omega$ and depends only on local $K_{\nu}$ norms of $V$.

Note that when the estimates (C1), (C2) depend only on the norms of $V_{-}$, that in (C3) depends on $V_{+}$also.

The proofs of (C2) and (C3) depend on a probabilistic solution for the Dirichlet problem associated to $H$. For these purposes it suffices to solve the problem for balls but actually a large array of regions are naturally accommodated. Let $B_{x}^{r} \equiv\{y|| y-x \mid \leqslant r\} ; \partial B_{x}^{r}=\{y|| x-y \mid=r\}$.

TheOREM C.1.4. Let $V \in K_{\nu}^{\text {loc }}$. Then for any compact $K$, there exists an $r_{0}$ so that, for any bounded continuous function $f$ on any $\partial B_{x}^{r}$ with $x \in K$ and $r \leqslant r_{0}$, there exists a unique continuous function $u$ on $B_{x}^{r}$ with (1) $u \uparrow \partial B_{x}^{r} \equiv f$, (2) $H u=0$ on $B_{x}^{r} \backslash \partial B_{x}^{r}$ in the sense that $(H \varphi, u)=0$ for any $C^{\infty} \varphi$ supported in the interior 
of $B_{x}^{r}$. Moreover $u$ is given by the "explicit" formula $u(y)=\left(M_{V} f\right)(y)$ with

$$
\left(M_{V} f\right)(y)=E_{y}\left(\exp \left(-\int_{0}^{T}(b(s)) d s\right) f(b(T))\right)
$$

with $T$ the stopping time

$$
T(b)=\inf _{t>0}\left\{b(t) \in \partial B_{x}^{r}\right\} .
$$

In addition, $r_{0}$ depends only on the $K_{\nu}$ norm of $V_{-}$restricted to a neighborhood of $K$.

For a complete proof, see [9]. The idea is the following: One first shows that by shrinking $r$ one can be sure that

$$
\sup _{x \in K} \sup _{y \in B_{x}^{r}} E_{y}\left(\int_{0}^{T\left(B_{x}^{r}\right)}\left|V_{-}(b(s))\right| d s\right)<1 .
$$

While $T$ is a random variable, it obviously "shrinks" with $r$; indeed, if $\nu \geqslant 3$ and $c_{\nu}|x-y|^{-(\nu-2)}$ is the kernel of $(-\Delta)^{-1}$, then (C6) will hold if

$$
\sup _{x \in K_{r}} \int_{|x-y|<r} c_{\nu}|x-y|^{-(\nu-2)}\left|V_{-}(y)\right| d^{\nu} y<1 .
$$

By Khas'minskii's lemma extended to stopping times, (C6) implies that $M_{V}$ is well defined as a map from $L^{\infty}\left(\partial B_{x}^{r}\right)$ to $L^{\infty}\left(B_{x}^{r}\right)$. That $M_{V} f(y) \rightarrow f$ as $y \rightarrow \partial B_{x}^{r}$ follows by extending the well-known argument for the case $V=0$ (see e.g. [155] for discussion of the $V=0$ case). Next, by exploiting the fact that $E_{y}\left(\int_{0}^{T} g(b(s)) d s\right)=\left[\left(H_{0}^{D}\right)^{-1} g\right](y)$ where $H_{0}^{D}$ is the Laplacian (time $\left.-\frac{1}{2}\right)$ in $B_{x}^{r}$ with vanishing boundary conditions, one shows that $u(y)=\left(M_{V} f\right)(y)$ obeys

$$
u(y)=E_{y}(f(b(T)))-\left[\left(H_{0}^{D}\right)^{-1} V u\right](y) .
$$

The first function on the right of (C7) is harmonic, so $-\frac{1}{2} \Delta u=-V u$, i.e. $u$ solves $H u=0$. Uniqueness comes from the fact that for $r$ small $H_{0}^{D}+V>0$.

The basic estimates (C2) and (C3) now follow from

TheOREM C.1.5 [9]. Fix $\lambda<1$. Under the hypotheses of Theorem C.1.4, we can pick $r$ so small, depending only on norms of $V_{-}$so that for any $f$

$$
\sup _{y \in B_{x}^{\lambda r}}\left|\left(M_{V} f\right)(y)\right| \leqslant C \int_{\partial B_{x}^{r}}|f(y)| d \sigma(y) .
$$

Moreover, by shrinking $r$ even further, depending on the local $K_{\nu}$-norm of both $V_{+}$and $V_{-}$, one can be sure that for any $f>0$

$$
\inf _{y \in B_{x}^{\lambda r}}\left(M_{V} f\right)(y) \geqslant C^{\prime} \int f(y) d \sigma(y)
$$

and that $(\mathrm{C} 8)$ holds for the same $r$.

Before describing (most of) the proof of (C8), (C9) we note that they prove (C2) and (C3). (C2) is a direct consequence of (C8). (C8) and (C9) together, imply that

$$
u(z) \leqslant C\left(C^{\prime}\right)^{-1} u(y)
$$


for any $z, y \in B_{x}^{\lambda r}$. To get from this to the result for any pair is a standard covering argument.

One of the nice features of the probabilistic approach is that (C9) follows from (C8)! For the same argument that led to (B24) implies that for $f \geqslant 0$

$$
\left(M_{V=0} f\right)(y)^{2} \leqslant\left(M_{V} f\right)(y)\left(M_{-V} f\right)(y) .
$$

Thus (C9) follows from (C8) for $M_{-V}$ and (C9) for $V=0$. The latter can be read off the standard Poisson kernel for $(-\Delta)$.

The proof of $(\mathrm{C} 8)$ directly mimics the proof of Theorem B.1.1. There is an analog of the semigroup property: if $f$ is given on $\partial B_{x}^{r}$, if $u$ is $M_{V} f$ for the $M_{V}$ associated to $B_{x}^{r}$ and if $g$ is $u$ restricted to $\partial B_{x}^{\tilde{r}}$ for $\tilde{r}<r$, then $\tilde{M}_{V} g$ is $u$ restricted to $B_{x}^{\tilde{r}}$. The only difficult step is the analog of Step 3 (which was trivial in Theorem B.1.1); $M_{V}$ is not selfdual. To prove $L^{1}$ to $L^{2}$ boundedness one must study a process which is Brownian motion run backwards from a hitting point. Khas'minskii's lemma and the Schwarz inequality then yields $L^{2}$ to $L^{\infty}$ boundedness of the adjoint of $M$. For details, see [9].

Open question. The above establishes the existence of an $L^{\infty}$ "Poisson kernel" for $H$. Study its continuity properties.

A main application of the bounds $(\mathrm{Cl})$ is to turn decay of $u$ in $L^{2}$ sense into decay pointwise; see §C3. As for Harnack's inequality, we will make an interesting application in $\S \mathrm{C} 8$. Another application is due to $\mathrm{M}$. and $\mathrm{T}$. Hoffman-Ostenhof and Simon [87, 88].

THEOREM C.1.6 $[87,88]$. Let $V \in K_{\nu}^{\text {loc }}$. Let $u$ be a real solution of $H u=0$. Then in the neighborhood of any zero of $u$, either $u$ changes sign or $u$ is identically zero.

Proof. By Harnack's inequality, if $u$ had one sign near $x$, with $u(x)=0$, then $u \equiv 0$ near $x_{0}$.

REMARK. In §C9, we discuss hypotheses which imply that $u$ cannot vanish in an open set without being identically zero.

Rauch [159] has noted that since a set which disconnects $R^{\nu}$ must have Hausdorff dimension [59] at least $\nu-1$, one has

CoROllary C.1.7 [159]. If $V \in K_{\nu}^{\text {loc }}$ and $u$ is a real solution of $H u=0$, then for any open set $\Omega$, either $\Omega \cap\{x \mid u(x)=0\}$ is empty or it has Hausdorff dimension at least $\nu-1$.

In the atomic case, $u$ is real analytic away from $S=\left\{x=\left(x_{1}, \ldots, x_{N}\right) \in\right.$ $R^{3 N} \mid x_{i}=x_{j}$ for some $i, j$ or $x_{i}=0$ for some $\left.i\right\}$ which has codimension 3 . It follows that the zero set has codimension at least 1 . Thus

Corollary C.1.8 [159]. In the atomic case $\left(\nu=3 N ; x=\left(x_{1}, \ldots, x_{N}\right)\right.$; $\left.V(x)=-Z \Sigma_{1}^{N}\left|x_{i}\right|^{-1}+\Sigma_{i-j}\left|x_{i}-x_{j}\right|^{-1}\right)$, the set of zeros of any real eigenfunction (which is not everywhere positive) has Hausdorff dimension precisely $3 N-1$ (even locally).

C2. Local estimates on $\nabla \varphi$. We have two goals in this section. First, we wish to show that the $L_{\text {loc }}^{2}$ bounds on $\nabla \varphi$ which follow from the results in $\S B 3$ for an eigenfunction in $L^{\infty}$ hold for any eigenfunction no matter what the growth 
at infinity. Secondly, we wish to show that the bounds only depend on local norms of $V_{-}$. This will be of some use in §C4. In this regard, we follow, in part, ideas of Sch'nol [174] who, however, required that $V_{-}$be bounded (!) since he did not exploit subsolution estimates.

LEMMA C.2.1. If $u \in L_{\mathrm{loc}}^{\infty}, \Delta u \in L_{\mathrm{loc}}^{1}$, then $\nabla u \in L_{\mathrm{loc}}^{2}$ and for any $\varphi$ in $C_{0}^{\infty}$

$$
\int \varphi(\nabla u)^{2} d^{\nu} x=\frac{1}{2} \int(\nabla \varphi) u^{2}-\int \varphi u \Delta u \text {. }
$$

In particular, if $\frac{1}{2} \Delta u=V u$, and $\varphi \geqslant 0$, then

$$
\int \varphi(\nabla u)^{2} d^{\nu} x \leqslant \frac{1}{2} \int(\Delta \varphi) u^{2}+2 \int\left(V_{-} \varphi\right) u^{2}
$$

Proof. It suffices to prove $(\mathrm{C} 10)$ for smooth $u$ and then use a standard mollifier argument. (C10) follows from two integrations by parts or equivalently from

$$
\operatorname{div}\left(\varphi u(\nabla u)-\frac{1}{2}(\nabla \varphi) u^{2}\right)=\varphi(\nabla u)^{2}+\varphi u \Delta u-\frac{1}{2}(\Delta \varphi) u^{2} .
$$

(C11) follows from (C10).

TheOREM C.2.2. Let $H u=0, V_{-} \in K_{\nu}^{\text {loc }}$. Then $\nabla u \in L_{\text {loc }}^{2}$ and for any compact $K$ and neighborhood $W$ of $K$

$$
\int_{K}|\nabla u|^{2} d^{\nu} x \leqslant C\left[\int_{W}|u(y)| d^{\nu} y\right]^{2}
$$

where $C$ only depends on the $K_{\nu}$-norm of $V_{-}$restricted to $W$ and on $K$ and $W$.

Proof. This follows directly from (C11) if we exploit (C1) by placing $K \subset W^{\prime} \subset \bar{W} \subset W$ with $W^{\prime}$ open and write (C1) in the form

$$
\sup _{x \in W^{\prime}}|u(x)| \leqslant C_{1} \int_{\bar{W}}|u(y)| d^{\nu} y
$$

and if we use the fact that $K_{\nu} \subset L_{\text {loc }}^{1}$.

We need the following in the section after next.

Corollary C.2.3. Let $V_{+} \in K_{\nu}^{\text {loc }}, V_{-} \in K_{\nu}$. Let $C_{r}$ be the hypercube $C_{r}=$ $\left\{x \in R^{\nu}|\max | x_{i} \mid \leqslant r\right\}$. Then for any solution of $\mathrm{Hu}=0$ and any integer $r$

$$
\int_{\left[C_{r+1} / C_{r}\right]}|\nabla u|^{2} d^{\nu} x \leqslant C \int_{\left[C_{r+2} / C_{r-1}\right]}|u(x)|^{2} d^{\nu} x
$$

where $C$ is r-independent (and only depends on $\left\|V_{-}\right\|_{K_{\nu}}$ ).

Proof. Let $K$ be a unit cube and $\tilde{K}$ a cube of side 3 centered at the center of K. By (C12)

$$
\int_{K}|\nabla u|^{2} d^{\nu} x \leqslant C_{1}\left[\int_{\tilde{K}}|u| d^{\nu} x\right]^{2} \leqslant C_{2} \int_{\tilde{K}}|u|^{2} d^{\nu} x .
$$

(C13) results by adding up these estimates for a partition of $C_{r+1} \backslash C_{r}$ into unit cubes. 
If we strengthen the hypotheses on $V$ by requiring the $V \in K_{\nu}^{(\alpha)}$ (defined in §B3) globally or locally, then by merely following the proof of Theorem B.3.5 and exploiting $(\mathrm{Cl})$ one finds that

THEOREM C.2.4. Let $V \in\left[K_{\nu}^{(\alpha)}\right]^{\text {loc }}$. Then any eigenfunction is locally in $C_{\alpha}$.

THEOREM C.2.5. Let $V \in K_{\nu}^{(\alpha)}$. Then for any solution $u$ of $H u=0$,

(a) If $\alpha<1$ and for any $x$

$$
\sup _{\{y|| y-x \mid \leqslant 1\}}\left\{|y-x|^{-\alpha}[|u(x)-u(y)|]\right\} \leqslant C \int_{|x-y| \leqslant 2}|u(y)| d^{\nu} y .
$$

(b) If $\alpha=1$

$$
\sup _{\{y|y-x| \leqslant 1\}}|(\nabla u)(y)| \leqslant C \int_{|x-y| \leqslant 2}|u(y)| d^{v} y .
$$

(c) If $\alpha>1$

$$
\sup _{\{y|| y-x \mid \leqslant 1\}}|x-y|^{-\alpha+1}[|(\nabla u)(y)-(\nabla u)(x)|] \leqslant C \int_{|x-y| \leqslant 2}|u(y)| d^{\nu} y .
$$

For more details on $\nabla u$ in the case of Coulomb Hamiltonians see Kato [110] and Hoffman-Ostenhof and Seiler [89].

C3. Decay of eigenfunctions. In this section, we discuss decay of solutions of $H u=E u$ with $u \in L^{2}$. One can presumably also study $L^{p}$-eigenfunctions. Since there exist continuous functions $u \in L^{2}$ which do not go to zero pointwise, the following is of some interest.

Theorem C.3.1. Let $V_{+} \in K_{\nu}^{\text {loc }}, V_{-} \in K_{\nu}$ and let $H u=E u$ with $u \in L^{2}$. Then $u(x) \rightarrow 0$ as $x \rightarrow \infty$.

REMARK. Since $u$ is equal a.e. to a continuous function (Theorem C.1.1), $u(x)$ makes good sense.

Proof. Since $u \in L^{2}, \lim _{x \rightarrow \infty} \int_{|x-y|<1}|u(y)|^{2} d y=0$. Thus by the Schwarz inequality, the limit is zero if $|u|^{2}$ is replaced by $u$. Thus, by Theorem C.1.2, $u$ goes pointwise to zero.

From this result and Theorem C.2.5, we immediately conclude that

THEOREM C.3.2. If $V \in K_{\nu}^{(1)}$ and $H u=E u$, then $\nabla u \rightarrow 0$ as $x \rightarrow \infty$.

Open question. The last theorem has global hypotheses, not only on $V_{-}$but also on $V_{+}$. Are there results on the pointwise decay of $\nabla u$ only requiring global restrictions on $V_{-}$? Note that by Corollary C.2.3, there is decay of the local $L^{2}$-norm of $\nabla u$ if $V_{-} \in K_{\nu}, V_{+} \in K_{\nu}^{\text {loc }}$.

There has developed a considerable literature on detailed estimates of decay of the eigenfunction $H u=E u$ with $E$ in the discrete spectrum of $H$; for example Agmon [2, 4], Ahlrichs [6], Ahlrichs et al. [7, 8], Bazley and Fox [20], Carmona [35], Carmona and Simon [37], Combes [40], Combes et al. [41], Combes and Thomas [43], Davies [45, 48], Deift et al. [50], Hoffman-Ostenhof [86, 91, 92, 94, 95], Hunziker [100], Lieb and Simon [130], Lithner [131], Mercuriev [134], Morgan [136], O'Connor [143], Sch'nol [174] (who had the 
earliest results), Sigal [177], Simon [180-182] and Slaggie and Wichmann [197]. Much of this involves detailed results on two special cases: (1) the $N$-body case and (2) the case where $V(x) \rightarrow \infty$ at infinity especially in a regular way (e.g. polynomials). Here, we will concentrate solely on those results which are applicable to general $V$. However, we call the reader's attention to some beautiful geometry ("Agmon metric") associated with the two special cases; see $[2,4,37,130,131]$.

While the first theorem below is a special case of the third, we state it separately for emphasis.

TheOREM C.3.3. Let $V_{-} \in K_{\nu}, V_{+} \in K_{\nu}^{\text {loc }}$. Suppose that $H$ has compact resolvent and let $H u=E u ; u \in L^{2}$. Then, for any $A>0$, there is a constant $C$ with

$$
|u(x)| \leqslant C e^{-A|x|} .
$$

TheOREM C.3.4. Let $V_{-} \in K_{\nu}, V_{+} \in K_{\nu}^{\text {loc }}$ and suppose $H u=E u$ where $E$ is in the discrete spectrum, i.e. $E$ is an isolated eigenvalue of finite multiplicity. Then for some $\delta>0$ and $C$

$$
|u(x)| \leqslant C e^{-\delta|x|} .
$$

TheOREM C.3.5. Let $V_{-} \in K_{\nu}, V_{+} \in K_{\nu}^{\text {loc }}$ and suppose $H u=E u$ where $E<\Sigma_{\text {ess }} \equiv \min \left[\sigma_{\text {ess }}(H)\right]$ with $\sigma_{\text {ess }}(H)$ the essential spectrum of $H$. Then for any $A$ with $\frac{1}{2} A^{2}<\Sigma_{\text {ess }}-E$, there is a $C$ so that (C15) holds.

REMARKS. 1. Sch'nol [174] proved Theorem C.3.3 if $V$ is bounded below. His proof actually works in general given the results we proved in $\S \mathrm{C} 2$. The proof we give follows Simon [181] (who used ideas of Combes and Thomas [43], see Remark 3 below).

2. For multiparticle systems, Theorem C.3.5 was proven by O'Connor [143] whose proof used the structure of $N$-body systems. Agmon [2] first noted that this bound actually holds for general $V$ 's.

3. The basic idea we exploit of considering the family $H(\vec{a})$ is due to Combes and Thomas [43]. Agmon [2] has an alternative proof which is more "elementary" in that only integration by parts is exploited rather than an operator analysis.

4. It has been noted in several places that one need not require $u \in L^{2}$. Typically, one can show that if $e^{-\rho(x)} \psi \in L^{2}$ for suitable $\rho$, then $e^{+\rho(x)} \psi \in L^{2}$; see especially Lithner [131] and Agmon [4] (and also [37, 182]).

The above results require two preliminary lemmas. The first is only required for Theorem C.3.5.

Lemma C.3.6 (Sigal [177]). Let $A=B+i C$ where $B, C$ are selfadjoint and $C$ is $B$-form bounded (i.e. A strictly sectorial). Then

$$
\inf \operatorname{Re} \sigma_{\text {ess }}(A) \geqslant \inf \sigma_{\text {ess }}(B) .
$$

Proof. Given $\varepsilon>0$, let $P$ be the spectral projection for $B$ for the interval $\left(-\infty, \Sigma_{\text {ess }}-\varepsilon\right)$ with $\Sigma_{\text {ess }}=\inf \sigma_{\text {ess }}(B)$. By definition, $P$ is finite rank. Then $A+\alpha P=B(1-P)+(B+\alpha) P+i C$ has numerical range in $N=\{z \mid \operatorname{Re} z$ $\left.>\Sigma_{\text {ess }}-\varepsilon\right\}$ for $\alpha$ sufficiently large. Since the spectrum is in the numerical 
range $\sigma_{\text {ess }}(A+\alpha P) \subset \sigma(A+\alpha P) \subset N$. By Weyl's theorem (see e.g. [162]) and the fact that $\lim _{\operatorname{Re} z \rightarrow-\infty}\left\|(A-z)^{-1}\right\|=0$, we have that $\sigma_{\text {ess }}(A) \subset N$.

LEMMA C.3.7 (“O'CONNOR's LEMMA" [143]). Let $U(\alpha)$ be a group parametrized by $R^{\nu}$. Let $P$ be a finite rank projection so that $P(\alpha)=U(\alpha) P U(\alpha)^{-1}$ has an analytic continuation from $R^{\nu}$ to $\left\{z \in C^{\nu}|| \operatorname{Im} z \mid<a\right\}$. Then for any $\psi \in$ $\operatorname{Ran} P, U(\alpha) \psi$ has an analytic continuation to the same region.

Proof. Let $\eta$ be an entire vector for $U(\alpha)$, i.e. $U(\alpha) \eta$ has a continuation to $C^{\nu}$. Since

$$
U(\alpha) P \eta=P(\alpha) U(\alpha) \eta
$$

for $\alpha$ real, we have the required result for the image under $P$ of the entire vectors. But since the entire vectors are dense and since $\operatorname{Ran} P$ is finite dimensional, this image is all of $\operatorname{Ran} P$.

Proof of Theorems C.3.3, 4, 5. Let $(U(\alpha) \psi)(x)=e^{i \alpha \cdot x} \psi(x)$. Then $U(\alpha) \psi$ has an analytic continuation to $|\operatorname{Im} \alpha|<M$ if and only if $e^{a|x|} \psi \in L^{2}$ for all $a<M$. If that is true then, by subsolution estimates $e^{a|x|} \psi \in L^{\infty}$. Thus, if $\psi$ is an eigenfunction of $H$, we need only prove that $U(\alpha) \psi$ has a continuation to $|\operatorname{Im} \alpha|<M$, with $M=\infty$ (C.3.3), $M>0$ (C.3.4), $M=\sqrt{2(\Sigma-E)}$ (C.3.5). Let $H(\alpha)$ be the operator $U(\alpha) H U(\alpha)^{-1}$ for $\alpha$ real. If $H(\alpha)$ has an analytic continuation to a neighborhood of $R^{\nu}$, any discrete eigenvalue $E\left(\alpha_{0}\right)$ of $H\left(\alpha_{0}\right)$ will move analytically for $\alpha$ near $\alpha_{0}$. But for $\left(\alpha-\alpha_{0}\right)$ real, $E(\alpha)=E\left(\alpha_{0}\right)$, since $H(\alpha)$ and $H\left(\alpha_{0}\right)$ are then unitarily equivalent so $E(\alpha)$ is constant and remains an eigenvalue so long as it stays away from $\sigma_{\text {ess }}(H(\alpha))$. This is an argument of Aguilar and Combes [5]. If we show that (1) $H(\alpha)$ has an analytic extension (in the same sense as Kato) to $|\operatorname{Im} \alpha|<M$, (2) $E \in \sigma_{\text {disc }}(H(\alpha)$ ) for all $\alpha$ in the strip, then standard eigenvalue perturbation theory $[114,162]$ will imply that

$$
P(\alpha)=(2 \pi i)^{-1} \int_{|E-z|=\varepsilon} d z(z-H(\alpha))^{-1}
$$

is analytic in the strip and the hypotheses of O'Connor's lemma are applicable. Thus, we are reduced to proving (1) and (2) above.

For $\alpha$ real

$$
H(\alpha)=H+\frac{1}{2} \alpha^{2}-\alpha \cdot p-p \cdot \alpha
$$

where $p=-i \nabla$. Since $Q(H)=Q\left(p^{2}\right), p$ is $H$-form bounded with relative bound zero so that $(\mathrm{C16})$ defines an entire analytic family of type (B) [114, 162]. Thus (1) holds. Since $E$ is assumed in $\sigma_{\text {disc }}(H)$ in all situations, by standard perturbation theory $[114,162], E$ is $\sigma_{\text {disc }}(H(\alpha))$ for $|\alpha| \leqslant \delta$ for some $\delta$ in all cases. Since $H(\alpha)$ and $H(i \operatorname{Im} \alpha)$ are unitarily equivalent,

$$
\sigma_{\text {disc }}(H(\alpha))=\sigma_{\text {disc }}(H(i \operatorname{Im} \alpha))
$$

and thus (2) is proven with $M=\delta$ in the context of Theorem C.3.4.

For Theorem C.3.3, we need only note that the resolvent of $H(\alpha)$ is analytic in $\alpha$ and by hypothesis compact for $\alpha$ real and thus compact for all $\alpha$, i.e. $\sigma_{\text {ess }}(H(\alpha))$ is empty proving (2) with $M=\infty$. 
For Theorem C.3.5, we use Lemma C.3.6 and (C17) to note that

$$
\begin{aligned}
\sigma_{\text {ess }}(H(\alpha)) & =\sigma_{\text {ess }}(H(i \operatorname{Im} \alpha)) \\
& \subset\left\{z \mid \operatorname{Re} z \in\left[\inf \sigma_{\text {ess }}\left(H-\frac{1}{2}|\operatorname{Im} \alpha|^{2}\right), \infty\right]\right\} \\
& =\left\{z \mid \operatorname{Re} z \in\left(\Sigma-\frac{1}{2}|\operatorname{Im} \alpha|^{2}, \infty\right)\right\}
\end{aligned}
$$

so $E \notin \sigma_{\text {ess }}(H(\alpha))$ if $E<\Sigma-\frac{1}{2}(\operatorname{Im} \alpha)^{2}$.

There are a number of natural questions about the relation of decay of eigenfunctions and the spectrum of $H$. A natural conjecture is "If $H \boldsymbol{u}=\boldsymbol{E u}$ and $|u(x)| \leqslant C e^{-\delta|x|}$, then $E \in \sigma_{\text {disc }}(H)$ "'. This conjecture is false! For example, if $b(t)$ is a typical Brownian path in one dimension, then $H=-d^{2} / d t^{2}+$ $\cos (b(t))$ has spectrum $[-1, \infty)$ but only eigenvalues with eigenfunctions which decay exponentially $[77,135,36]$. That is, random potentials present examples with nondiscrete eigenvalues whose eigenfunctions decay exponentially.

But the following is still open.

Open question. If $V_{-} \in K_{\nu}, V_{+} \in K_{\nu}^{\text {loc }}$ and $H u=E u$ where $|u(x)| \leqslant C e^{-a|x|}$ for all $a$, does it follow that $H$ has compact resolvent?

An equivalent form is: if $H$ does not have compact resolvent, is it true that for some $a>0, e^{a|x|} u \notin L^{2}$ ? In this form, an attack on this problem (for some special $V$ 's) was begun by Froese et al. [60]. Typical of their results is

THEOREM C.3.8. Suppose that $V \in K_{\nu}$ and that $W \equiv(x \cdot \nabla) V$ (distributional gradient) is also in $K_{\nu}$. Then for any eigenfunction $u$ of $H_{0}$ there is an a so that $e^{a|x|} u$ is not in $L^{2}$.

SKETCH OF PROOF. We sketch the proof ignoring all domain questions which are fully treated in [60]. Let $\vec{D}$ be the gradient operator and let

$$
A=\frac{1}{2}(\vec{x} \cdot \vec{D}+\vec{D} \cdot \vec{x}), \quad B=\frac{1}{2}\left(r^{-1} \vec{x} \cdot \vec{D}+\vec{D} \cdot \vec{x} r^{-1}\right)
$$

(where $r=|x|$ ) so both $A$ and $B$ are skewadjoint and

$$
B=r^{-1}\left(A-\frac{1}{2}\right)
$$

since $\left[r^{-1}, D \cdot x\right]=r^{-1}$. Suppose $H \psi=E \psi$ and that $\psi_{a} \equiv e^{a|x|} \psi$ is in $L^{2}$. Then, by formal calculations,

$$
e^{a r} H e^{-a r} \psi_{a}=E \psi_{a}, \quad e^{a r} H e^{-a r}=H-a B-\frac{1}{2} a^{2} \equiv H_{a} .
$$

Moreover (and here the domain problems are nontrivial since $\psi_{a}$ may not be a priori in the domain of $A$ ),

$$
\left(\psi_{a}, A H_{a} \psi_{a}\right)=E\left(\psi_{a}, A \psi_{a}\right)=\left(H_{a} \psi_{a}, A \psi_{a}\right)=\left(\psi_{a}, H_{a}^{*} A \psi_{a}\right)
$$

so, by (C17a)

$$
\left(\psi_{a},[A, H] \psi_{a}\right)=2 a \operatorname{Re}\left(\psi_{a}, A B \psi_{a}\right)=2 a\left(\psi_{a}, A r^{-1} A \psi_{a}\right) \geqslant 0 .
$$

Thus, $\left(\psi_{a},\left(-2 H_{0}+W\right) \psi_{a}\right) \geqslant 0$. Since $(\mathrm{C} 17 \mathrm{~b})$ and the symmetry of $i B$ imply that $\left(\psi_{a},\left(H_{0}-E+V-\frac{1}{2} a^{2}\right) \psi_{a}\right)=0$, we conclude that

$$
\left(\psi_{a},\left(H_{0}-V-W+E\right) \psi_{a}\right) \leqslant-\frac{1}{2} a^{2}\left(\psi_{a}, \psi_{a}\right) \text {. }
$$

Since $H_{0}-V-W$ is bounded below by the $K_{\nu}$-hypotheses, this cannot happen for any sufficiently large $a$. 
C4. Eigenfunctions and spectrum. In this section, we prove that

TheOREM C.4.1 [174, 190]. Let $V_{+} \in K_{\nu}^{\text {loc }}, V_{-} \in K_{\nu}$. Let $H u=E u$ where $u$ is polynomially bounded. Then $E \in \operatorname{spec}(H)$.

REMARKS. 1. This was first proven by Sch'nol [174] who assumed that $V$ is bounded below. We will show here that given our preliminaries in $\S \mathrm{C} 2$, his proof extends to general $V$. Simon [190] proved the general case by a different method. Simon was unaware of Sch'nol's work.

2. In the next section we will prove a kind of converse of this result. It is not true that if $E \in \operatorname{spec}(H)$, then $H u=E u$ has a polynomially bounded solution, but at least

$$
\sigma(H)=\overline{\{E \mid H u=E u \text { has a polynomially bounded solution }\}}
$$

where the overbar denotes closure (see Corollary C.5.5).

3. All the proofs actually show that if for all $A>0,|u(x)| \leqslant C e^{A|x|}$, then $E \in \operatorname{spec}(H)$.

FIRST PROOF [190]. $u$ is clearly in $L_{-\delta}^{2}$ for some $\delta$ so that $E$ is an eigenvalue of $H_{-\delta}$ and thus in $\operatorname{spec}\left(H_{-\delta}\right)$. But $\operatorname{spec}\left(H_{-\delta}\right)=\operatorname{spec}(H)$ (Theorem B.6.4).

SECOND PROOF (FOLLOWING AND EXTENDING [174]). Let $C_{r}, r=1,2, \ldots$, be the hypercube $\left\{x \in R^{\nu}|\max | x_{i} \mid \leqslant r\right\}$, let

$$
F(r)=\int_{C_{r}}|u(x)|^{2} d^{\nu} x
$$

and let $j_{r}$ be a function in $C_{0}^{\infty}$ which is supported in $C_{r+1}$ and is 1 in $C_{r}$. We can choose $j_{r}$ so $0 \leqslant j$ and so that for any fixed multi-index $\alpha, \sup _{r, x}\left|\left(D^{\alpha} j_{r}\right)(x)\right|$ $<\infty$. We let $w_{r}=j_{r} u /\left\|j_{r} u\right\|_{2}$. We will show that for suitable $r_{n} \rightarrow \infty$ we have that $\left\|(H-E) w_{r_{n}}\right\| \rightarrow 0$ which implies that $E \in \operatorname{spec}(H)$.

If $F(r+3) \geqslant a F(r)$ for some $a>1$ and all $r \geqslant r_{0}$, then $F\left(r_{0}+3 k\right) \geqslant a^{k} F\left(r_{0}\right)$ which is inconsistent with polynomial boundedness of $u$. Thus, we can find $r_{n}$ with

$$
F\left(r_{n}+2\right) / F\left(r_{n}-1\right) \rightarrow 1
$$

Note that

$$
(H-E) j_{r} u=j_{r}(H-E) u+\left[H, j_{r}\right] u=-\frac{1}{2}\left(\Delta j_{r}\right) u-\left(\nabla j_{r}\right)(\nabla u) .
$$

Thus, since $\left\|\Delta j_{r}\right\|_{\infty}$ and $\left\|\nabla j_{r}\right\|$ are uniformly bounded

$$
\left\|(H-E) j_{r} u\right\|^{2} \leqslant C_{1} \int_{C_{r+1} \backslash C_{r}}\left[|u|^{2}+|\nabla u|^{2}\right] d^{\nu} x \leqslant C_{2} \int_{C_{r+2} \backslash C_{r-1}}|u|^{2} d^{\nu} x
$$

by Corollary A.2.3. Thus

$$
\begin{aligned}
\left\|(H-E) w_{r}\right\|^{2} & \leqslant\left[F\left(r_{n}+2\right)-F\left(r_{n}-1\right)\right] / F\left(r_{n}\right) \\
& \leqslant\left[F\left(r_{n}+2\right) F\left(r_{n}-1\right)^{-1}\right]-1
\end{aligned}
$$

goes to zero by $(\mathrm{C} 18)$ as required. 
Notice in the above proof that if $F(r) \rightarrow \infty$ at $\infty$, then $w_{r}$ goes weakly to zero so that we have

THEOREM C.4.2 [174, 190]. If $V_{+} \in K_{\nu}^{\mathrm{loc}}, V_{-} \in K_{\nu}$ and $H u=E u$ has a polynomially bounded solution which is not in $L^{2}$, then $E \in \sigma_{\mathrm{ess}}(H)$.

C5. Eigenfunction expansions. In this section, we discuss a general eigenfunction expansion for $H$ and related objects like the Radon-Nikodym derivatives $d E_{\lambda} / d \rho(\lambda)$ of the spectral projection measure. In the context of general elliptic differential opeators with nice coefficients, these ideas were developed by a variety of authors, most notably Berezanskii [22], Browder [32], Garding [67], Gel'fand [69] and Kac [107]; see Berezanskii's book [23] for further details. Herbst and Sloan [83] emphasized the significance of $L^{p}$ estimates on $e^{-H}$ to prove the applicability of this expansion to general Schrödinger operators including ones with rather singular potentials. Further developments along these lines appear in Kovalenko and Semenov [123], whose discussion we follow in part here.

We dub the expansion that occurs here a BGK expansion. We begin by saying a few words about the somewhat weaker information implicit in the spectral theorem and the much stronger information in the expansions associated with the work of Ikebe [101] and Povzner $[157,158]$ which we dub IP expansions.

Let $A$ be a general selfadjoint operator on $L^{2}\left(R^{\nu}, d^{\nu} x\right)$ which we suppose has simple spectrum to avoid carrying along an extra index. Given a cyclic vector $\varphi$ for $A$, we can form the spectral measure $d \mu_{\varphi}$ on $R$ by $\left(\varphi, e^{-i t A} \varphi\right)=$ $\int e^{-i E t} d \mu_{\varphi}(E)$. The spectral theorem asserts that there exists a unitary operator $U: L^{2}\left(R^{\nu}, d^{\nu} x\right) \rightarrow L^{2}\left(R, d \mu_{\varphi}\right)$ so that $U A U^{-1}$ is multiplication by $x$. Since $U$ clearly maps $\delta\left(R^{\nu}\right)$ into $\delta^{\prime}(R)$, it has a distributional kernel $K(E, y), E \in R$, $y \in R^{\nu}$, by the nuclear theorem of Schwarz [160]. Explicitly, for all $F \in \delta\left(R^{\nu}\right)$ and $g \in \mathcal{S}(R)$, we have that

$$
(g(H) \varphi, F)=K(g \otimes F) .
$$

If $A$ is a differential operator with smooth coefficients, it is easy to see that $K$ obeys $(A-E) K(E, y)=0$ in distributional sense, so in some sense $K(E, \cdot)$ provides eigenfunctions of $A$. Of course, this object may not be defined for fixed $E$.

In contrast, the BGK expansion will construct honest eigenfunctions, $\varphi(y, E)$, for a.e. $E$ with respect to the spectral measure class (defined below) and these eigenfunctions will be polynomially bounded. Moreover, there will be an eigenfunction expansion, which in the case of simple spectrum reads (for a suitable dense set of $f$ 's)

$$
(f, g(H) f)=\int d \rho(E) g(E)|\tilde{f}(E)|^{2}
$$

where

$$
\tilde{f}(E)=\int \overline{\varphi(y, E)} f(y) d^{\nu} y .
$$


For the case when $V$ decays sufficiently rapidly at infinity one has an IP expansion. There are bounded eigenfunctions $\varphi(y, k)$ indexed by $k \in R^{\nu}$ and $L^{2}$-eigenfunctions $\left\{\varphi_{n}(y)\right\}_{n=1}^{N}$ (where $N$ may be infinite) so that

$$
(f, g(H) f)=\sum_{n=1}^{N} g\left(E_{n}\right)\left|\tilde{f}_{n}\right|^{2}+\int|\tilde{f}(k)|^{2} g\left(k^{2}\right) d^{\nu} k
$$

with $\tilde{f}_{n}=\left(\varphi_{n}, f\right)$ and $\tilde{f}(k)=\int \overline{\varphi(y, k)} f(y) d^{\nu} y$. One critical difference between $(\mathrm{C} 21)$ and $(\mathrm{C} 19)$ is the explicitness of the measure $d^{\nu} k$. This has important spectral consequences: it implies that $H$ has no singular spectrum in that case. Another difference is that in the IP case, the $\varphi$ are constructed by an explicit method which links them to scattering theory. There are related expansions for certain long range two-body potentials (see e.g. [3, 102-104, $117,168]$, for one-dimensional periodic potentials $[69,51]$ and for higherdimensional periodic potentials $[69,186,206])$.

Because one knows a priori little about the measure $d \rho$ in (C19), the BGK expansions appear to give little practical information beyond that already in the spectral theorem. For this reason, some workers, myself included, regarded them for many years as having little significance. The work of Pastur [148], who used critically the polynomial boundedness of the $\varphi$ in the BGK expansion to prove that certain $H$ with random potentials have no absolutely continuous spectrum, changed this attitude. More recently, Avron and Simon $[18,19]$ have used the same ideals to prove the absence of a.c. spectrum in certain $H$ with almost periodic potentials.

Definition. Two Borel measures on $R$ are called equivalent if and only if they are mutually absolutely equivalent. A measure class is an equivalence class under this relation. Given a selfadjoint operator, $A$, with spectral projections $E(\Delta)$, pick an orthonormal basis $\left\{\varphi_{n}\right\}_{n=1}^{\infty}$ and a sequence $a_{n}$ with $a_{n}>0$, $\Sigma a_{n}<\infty$. The measure $\mu(\cdot)=\Sigma a_{n}\left(\varphi_{n}, E(\cdot) \varphi_{n}\right)$ has $\mu(\Delta)=0$ if and only if $E(\Delta)=0$. Thus, the measure class of $\mu$ is independent of $\left\{\varphi_{n}\right\}$ and $\left\{a_{n}\right\}$ and is associated to $A$. It is called the spectral measure class for $A$. Any measure $\mu$ in this class is called a spectral measure for $A$.

The BGK eigenfunction expansion comes most naturally out of the following "trace class Radon-Nikodym theorem":

THEOREM C.5.1. Suppose that for each bounded Borel set $\Delta \subset R$, we are given a nonnegative trace class operator $A(\Delta)$ on a fixed separable Hilbert space $\mathcal{H}$, and that if $\Delta=\cup_{n=1}^{\infty} \Delta_{n}$ with $\Delta_{i} \cap \Delta_{j}=\varnothing$ and $\Delta$ bounded, then $A(\Delta)=$ $s$-lim $\Sigma_{1}^{m} A\left(\Delta_{n}\right)$. Then, there exists an ordinary Borel measure $d \rho$ and a positive trace class operator-valued measurable function $A(\lambda)$, so that

$$
\begin{aligned}
& \text { (i) } A(\Delta)=\int_{\Delta} A(\lambda) d \rho(\lambda), \\
& \text { (ii) } \operatorname{Tr}(A(\lambda))=1 \quad \text { a.e. } d \rho,
\end{aligned}
$$

where $(\mathrm{C} 22)$ is intended in weak sense, i.e. $(\varphi, A(\Delta) \varphi)=\int(\varphi, A(\lambda) \varphi) d \rho$ for all $\varphi$. These two conditions fix $\rho$ and $A(\lambda)$.

Proof. Without loss, suppose that $\mathcal{H}=l_{2}[1, \infty)$ with standard basis $\left\{e_{n}\right\}_{n=1}^{\infty}$. Let $\rho(\Delta)=\operatorname{Tr}(A(\Delta))$. It is easy to see that $\rho$ is a Borel measure. For fixed $i, j$, 
let $\mu_{i, j}(\Delta)=\left(\varphi_{i}, A(\Delta) \varphi_{j}\right)$. It is easy to see that each $\mu_{i, j}$ is a signed measure, that $\mu_{i, i} \geqslant 0$, that $\Sigma \mu_{i, i}=\rho$ and that for each finite sequence of rationals $\left\{\alpha_{i}\right\}_{i=1}^{N}$, we have that $\Sigma \bar{\alpha}_{i} \alpha_{j} \mu_{i, j}(\Delta) \geqslant 0$. Moreover, if $\rho(\Delta)=0$, then $\mu_{i, j}(\Delta)=$ 0 . By the ordinary Radon-Nikodym theorem, $\mu_{i, j}(\Delta)=\int a_{i j}(\lambda) d \rho(\lambda)$. Moreover, for all $\lambda$, all $i, j$ and all rational sequences, $\Sigma \bar{\alpha}_{i} \alpha_{j} a_{i j}(\lambda) \geqslant 0, \Sigma_{j} a_{j j}(\lambda)=1$. Thus, the $a_{i j}(\lambda)$ are matrix elements of a positive trace class operator $A(\lambda)$ with $\operatorname{Tr}(A(\lambda))=1$. By construction (C22) holds.

We will get the eigenfunctions via an object of independent interest.

THEOREM C.5.2. Let $V_{+} \in K_{\nu}^{\text {loc }}, V_{-} \in K_{\nu}$ and let $H=-\frac{1}{2} \Delta+V$. Fix $\delta>\frac{1}{2} \nu$. There exists a spectral measure $d \rho(\lambda)$ and for a.e. $\lambda$ (with respect to $\rho$ ) a function $F(x, y ; \lambda)$ on $R^{\nu} \times R^{\nu}$ so that

(i) $F(x, y ; \lambda)$ is jointly measurable in $x, y, \lambda$ and for fixed $\lambda$, jointly continuous in $x, y$.

(ii) For a.e. $\lambda, \int|F(x, y ; \lambda)|^{2}\left(1+x^{2}\right)^{-\delta}\left(1+y^{2}\right)^{-\delta} d^{\nu} x d^{\nu} y \leqslant 1$.

(iii) $|F(x, y ; \lambda)| \leqslant C\left(1+x^{2}\right)^{\delta / 2}\left(1+y^{2}\right)^{\delta / 2}$.

(iv) For any bounded Borel function, $g$, on $R$ and any $\varphi, \psi \in L_{\delta}^{2}$

$$
(\varphi, g(H) \psi)=\int g(\lambda)\left[\int F(x, y ; \lambda) \overline{\varphi(x)} \psi(y) d^{\nu} x d^{\nu} y\right] d \rho(\lambda)
$$

(v) For any fixed $y,\left(H_{x}-\lambda\right) F(\cdot, y ; \lambda)=0$, in distributional sense.

Remarks. 1. By (ii), the integrand in [ $\cdots]$ in (C23) is absolutely integrable and the integral is a bounded function.

2. (C23) shows that the combination $F(x, y ; \lambda) d \rho(\lambda)$ is intrinsic to $H$. Both $d \rho$ and $F$ depend on a choice.

3. Because of (C23) we will henceforth denote $F$ by $d E(x, y ; \lambda) / d \rho$. It is, in a very real sense, an integral kernel of the $R-N$ derivative of the spectral projection although it is not an integral kernel in the sense of defining an operator on $L^{2}$; only from $L_{\delta}^{2}$ to $L_{-\delta}^{2}$.

Proof. By Theorem B.9.1, for any bounded $\Delta, E(\Delta)\left(1+x^{2}\right)^{-\delta / 2}$ is Hilbert-Schmidt, so $A(\Delta) \equiv\left(1+x^{2}\right)^{-\delta / 2} E(\Delta)\left(1+x^{2}\right)^{-\delta / 2}$ is trace class and it is easy to see it is a measure. Thus, by Theorem C.5.1, there exists a trace class operator, $a(\lambda)$, with kernel $a(x, y ; \lambda)$ so that $A(\Delta)=\int a(\lambda) d \rho(\lambda)$ where

$$
\rho(\Delta)=\operatorname{Tr}\left(\left(1+x^{2}\right)^{-\delta / 2} E(\Delta)\left(1+x^{2}\right)^{-\delta / 2}\right) .
$$

By (C24), it is easy to see that $\rho$ is a spectral measure. We define $F(x, y ; \lambda)=$ $\left(1+x^{2}\right)^{\delta / 2} a(x, y ; \lambda)\left(1+y^{2}\right)^{\delta / 2}$, initially a.e. Then measurability is evident as are (ii) and (iv) for $g$ 's of compact supports (by a limiting argument from $g$ 's which are finite linear combinations of characteristic functions).

By Theorem B.6.3, $e^{-t H}$ maps $L_{-\delta}^{2}$ to $L_{-\delta}^{2}$. Moreover, if $\tilde{H}$ is the map $-\frac{1}{2} \Delta_{x}-\frac{1}{2} \Delta_{y}+V(x)+V(y)$ on $L^{2}\left(R^{2 \nu}\right)$, then $e^{-t \tilde{H}}$ maps $L_{-2 \delta}^{2}$ to $L_{-2 \delta}^{2}$. By (ii), $F(\cdot, \cdot ; \lambda) \in L_{-2 \delta}^{2}$. We claim that $e^{-\tilde{H}} F(\cdot, \cdot ; \lambda)=e^{-2 \lambda} F(\cdot, \cdot ; \lambda)$ which will imply that $F$ is locally bounded on account of Theorem B.6.3 again. For, by definition of $\tilde{H}$

$$
\int\left(e^{-\tilde{H}} F\right)(x, y ; \lambda) \overline{\varphi(x)} \psi(y) d^{\nu} x d^{\nu} y=\int F(x, y ; \lambda) \overline{\left(e^{-H} \varphi\right)}(x)\left(e^{-H} \psi\right)(y)
$$


so for any bounded $g$ of compact support, any $\varphi, \psi$

$$
\int g(\lambda)\left[\int\left\{e^{-\tilde{H} F}-e^{-2 \lambda} F\right\}(x, y ; \lambda) \overline{\varphi(x)} \psi(y) d^{\nu} x d^{\nu} y\right] d \rho(\lambda)=0
$$

which implies the required result for a.e. $\lambda$.

Similarly $e^{-t \hat{H}} F=e^{-2 t \lambda} F$ for all rational $t$. It follows that $(\tilde{H}-2 \lambda) F=0$ in distributional sense so that $F$ is jointly continuous and (iii) follows from (ii) and Harnack's inequality.

In the same way, exploiting $e^{-t H_{x}}$, we see that for any $\psi \in C_{0}^{\infty}$,

$$
\left(H_{x}-\lambda\right)\left[\int F(x, y ; \lambda) \Psi(y) d^{\nu} y\right]=0
$$

in distribution sense, so that by continuity of $F$, we obtain (v).

In $\S \mathrm{B} 12$, we proved that when $H=H_{0}+V+W$ where $W$ obeys (B39) with $\mu \leqslant 1$ and $V_{+} \in K_{\nu}^{\text {loc }}, V_{-} \in K_{\nu}$, then $\left(1+|x|^{2}\right)^{-\delta / 2} E(\Delta)\left(1+x^{2}\right)^{-\delta / 2}$ is trace class so long as $\delta>\nu$. In that case, one can construct an $F$ obeying (i), (ii), (iv) by just following the above proof. However, the proof of pointwise bounds above exploited the semigroup and will not work in this case. Another method may well work in general but if $V \in L_{\text {loc }}^{2}$ one can easily show that $F$ is a distributional solution of $(\tilde{H}-2 \lambda) F=0$ and thus obtain (iii), (v) (since $F$ is a priori only in $L_{\text {loc }}^{2}, \tilde{V} F$ may not be defined if we don't know that $\tilde{V}$ is in $L_{\text {loc }}^{2}$ ). Thus

THEOREM C.5.3. Let $W$ obey (B39) with $\mu \leqslant 1$. Let $V_{+} \in K_{\nu}^{\text {loc }}, V_{-} \in K_{\nu}$ and let $H=-\frac{1}{2} \Delta+V+W$. Fix $\delta>\nu$. There exists a spectral measure $d \rho(\lambda)$ and for a.e. $\lambda$ (with respect to $\rho$ ) a function $F(x, y ; \lambda)$ on $R^{\nu} \times R^{\nu}$ so that (i), (ii), (iv) of Theorem C.5.2 holds. If moreover, $V \in L_{\mathrm{loc}}^{2}$, then (iii), (v) of that theorem also hold.

We note now that the considerations we are about to give for $W=0$ can be made also in this more general case.

Returning to the context of Theorem C.5.2, let $N(\lambda)$ be the rank of $a(\lambda)$. It is not hard to see that this is a measurable function of $\lambda$. By the usual expansion relation for Hilbert-Schmidt operators, there are for each $\lambda, N(\lambda)$, linearly independent functions $\left\{f_{j}(x ; \lambda)\right\}_{j=1}^{N(\lambda)}$ so that

$$
a(x, y ; \lambda)=\sum_{j=1}^{N(\lambda)} f_{j}(x ; \lambda) \overline{f_{j}(y ; \lambda)}
$$

where (C25) holds in the sense that

$$
\int \overline{\varphi(x)} \psi(y) a(x, y ; \lambda) d x d y=\sum_{j=1}^{N(\lambda)}\left(\varphi, f_{j}(\lambda)\right)\left(f_{j}(\lambda) \psi\right) .
$$

Normally, one writes (C25) with the $f_{j}$ 's orthonormal and an extra factor of $\alpha_{j}$ added. In (C25), we have orthogonal $f_{j}$ but they are not normalized; indeed

$$
\sum_{j=1}^{N(\lambda)}\left\|f_{j}(\lambda)\right\|_{2}^{2}=1
$$


If $a(\lambda)$ has simple spectrum, the $f$ 's are uniquely determined. If there is some degenerate spectrum, some choice is involved. It is not hard to see that the choice can always be made so that $f_{j}(\lambda, x)$ is jointly measurable in $\lambda, x$ (on the measurable set where $N(\lambda) \geqslant j$ ). If we now define

$$
\varphi_{j}(x, \lambda)=\left(1+x^{2}\right)^{\delta / 2} f_{j}(x, \lambda), \quad \Delta_{n}=\{\lambda \mid N(\lambda)=n\},
$$

we have

TheOREM C.5.4. Let $V_{+} \in K_{\nu}^{\text {loc }}, V_{-} \in K_{\nu} ; H=-\frac{1}{2} \Delta+V$. Fix $\delta>\nu / 2$. Then, there exist a spectral measure $d \rho(x)$ and a family of disjoint measurable sets $\left\{\Delta_{n}\right\}_{n=1}^{\infty}$ whose union supports $\rho$ so that if $\lambda \in \Delta_{n}$, there are $n$ functions $\left\{\varphi_{j}(x, \lambda)\right\}_{j=1}^{n}$ obeying

(i) $(H-\lambda) \varphi_{j}=0$ in distributional sense.

(ii) $\left|\varphi_{j}(x, \lambda)\right| \leqslant C\left(1+x^{2}\right)^{\delta / 2}$.

(iii) For $\lambda$ fixed, the $\left\{\varphi_{j}\right\}_{1}^{n}$ are linearly independent.

(iv) For any $f \in L_{\delta}^{2}$, define bounded functions $\left(U_{j} f\right)(\lambda)$ for $j \leqslant N(\lambda)$ by

$$
\left(U_{j} f\right)(\lambda)=\int \overline{\varphi_{j}(x, \lambda)} f(x) d^{\nu} x .
$$

Then

$$
\int d \rho(\lambda) \sum_{j=1}^{N(\lambda)}\left|\left(U_{j} f\right)(\lambda)\right|^{2}=\|f\|^{2} .
$$

(v) $U$ extends to a unitary map of $L^{2}\left(R^{\nu}, d^{\nu} x\right.$, onto $\mathcal{K} \equiv \oplus_{n=1}^{N}$ $L^{2}\left(R, \mathbf{C}^{n}, \mathfrak{X}_{n} d \rho\right)$ with $\mathcal{X}_{n}$ the characteristic function of $\Delta_{n}$. Explicitly

$$
\left(U_{j} f\right)(\lambda)=\text { 1.i.m. } \int_{|x| \leqslant R} \overline{\varphi_{j}(x, \lambda)} f(x) d^{\nu} x
$$

where 1.i.m. means limit in FG-norm.

(vi) If $g$ is a bounded Borel function and $f \in L^{2}\left(R^{\nu}, d^{\nu} x\right)$, then

$$
U[g(H) f]=g(\lambda)(U f) .
$$

(vii) If $f \in D(H)$, then $U(H f)=\lambda U f$. (viii) If $g \in \mathcal{H}$,

$$
\left(U^{-1} g\right)(x)=\underset{|E| \rightarrow \infty, N \rightarrow \infty}{\operatorname{lim.m.}} \int_{|\lambda| \leqslant E} \sum_{j=1}^{\min (N, N(\lambda))} g_{j}(\lambda) \varphi_{j}(x, \lambda) d \rho(\lambda)
$$

where 1.i.m. means convergence in $L^{2}$-norm.

Proof. Pick $N, \rho, \varphi$ as discussed before the theorem. Thus (iii) holds and $\varphi$ obeys

$$
\int_{|x-y|<1}\left|\varphi_{j}(x, \lambda)\right|^{2} d x \leqslant C\left(1+y^{2}\right)^{\delta / 2}
$$

Moreover, by the definition of $F$ and the fact that the $f$ are eigenfunctions of $a$,

$$
\varphi_{j}(x, \lambda)=\int F(x, y ; \lambda)\left(1+y^{2}\right)^{-\delta} \varphi_{j}(y, \lambda) d^{\nu} y
$$


so by (i), (iii) and (v) of Theorem C.5.2, the $\varphi_{j}$ 's obey (i) of this theorem and then (C31), (ii) holds.

Again by the definition of $F$, by (C23) and by (C25) we have that for any $f \in L_{\delta}^{2}$ :

$$
(f, G(H) f)=\int d \rho(\lambda) G(\lambda) \sum_{j=1}^{N(\lambda)}\left|\left(U_{j} f\right)(\lambda)\right|^{2}
$$

where $U$ is given by (C27). This formula with $C \equiv 1$ proves (C28) and thus (iv) is proven.

Given (C27), $U$ is easily seen to extend to an isometry from $L^{2}$ to $\mathcal{H}$ given by $\left(\mathrm{C} 27^{\prime}\right)$. The remaining assertion in (v) is that $U$ is onto.

As a preliminary, we note that since $e^{-t H_{x}} F(\cdot, y ; \lambda)=e^{-t \lambda} F$, we have that as a map from $L_{-\delta}^{2}$ to $L_{-\delta}^{2}, e^{-t H_{x}} \varphi=e^{-t \lambda} \varphi$ so

$$
U\left(e^{-t H} f\right)=e^{-t \lambda} U f
$$

initially for $f \in L_{\delta}^{2}$ and then for all $L^{2}$ by a limiting argument. Since any continuous function in [inf $\operatorname{spec}(H), \infty)$ vanishing at $\infty$ is a uniform limit of polynomials in $e^{-t \lambda}$, (C29) holds for such $g$ 's and then by a limiting argument for all bounded Borel $g$ 's, i.e. (vi) is proven.

Now, we return to (v) and suppose that $h \in \mathcal{H}$ is orthogonal to $\operatorname{Ran} U$. By (C26) and Harnack's inequality for all $\lambda$

$$
\sum_{j=1}^{N(\lambda)}\left|\varphi_{j}(x, \lambda)\right|^{2} \leqslant C\left(1+x^{2}\right)^{\delta / 2}
$$

Since $h \in \mathcal{H}$, we have $\Sigma_{1}^{N(\lambda)}\left|h_{j}(\lambda)\right|^{2}<\infty$ for a.e. $\lambda$ so for a.e. $\lambda$, we can define $k(x, \lambda) \equiv \Sigma_{j=1}^{N(\lambda)} h_{j}(\lambda) \varphi_{j}(x, \lambda)$ and for any $f \in C_{0}^{\infty}\left(R^{\nu}\right)$ and $g \in C_{0}^{\infty}(R)$

$$
\int f(x) g(\lambda) \overline{k(x, \lambda)} d \rho(\lambda) d^{\nu} x=(h, U g(H) f)=0
$$

since sums and integrals can be freely interchanged. It follows that $k \equiv 0$ for a.e. $x, \lambda$, and so a.e. $\lambda(d \rho),\left(1+x^{2}\right)^{-\nu} k(\cdot, \lambda)=0$ as an element of $L^{2}$. But $\left\{\left(1+x^{2}\right)^{-\nu / 2} \varphi_{j}\right\}$ are orthogonal in $L^{2}$ and $\left\|\left(1+x^{2}\right)^{-\nu / 2} \varphi_{j}\right\| \neq 0$, so $\sum h_{j}(\lambda) \varphi_{j}(x, \lambda)=0$ implies $h_{j}(\lambda)=0$, a.e. $d \rho$. Thus $U$ is onto. (viii) then follows easily and (vii) follows by a limiting argument from (vi).

Theorem C.5.4 and the fact that if $\lambda_{0} \in \operatorname{spec}(H)$ then $\rho\left(\lambda_{0}-\varepsilon, \lambda_{0}+\varepsilon\right)>0$ for all $\varepsilon$ together imply that if $\lambda_{0} \in \operatorname{spec}(H)$, then for $\lambda^{\prime}$ arbitrarily close to $\lambda_{0}$, $H \varphi=\lambda^{\prime} \varphi$ has polynomially bounded solutions. Thus

COROLlaRY C.5.5. $\sigma(H)$ is the closure of the set of $\lambda$ for which $H \varphi=\lambda \varphi$ has a polynomially bounded solution.

Open question. Does Corollary C.5.5 have a version with multiplicities? Explicitly, the set $\Delta_{n}$ is uniquely determined a.e. $\rho$ by the fact that $H$ has multiplicity $n$ there. Is it true that if $H u=E u$ has $n$ linearly independent solutions, then for all $\varepsilon, \sum_{j=n}^{\infty} \rho\left((E-\varepsilon, E+\varepsilon) \cap \Delta_{j}\right)>0$ ?

As we just remarked

COROLlaRY C.5.6. The spectral multiplicity of $H$ on $\Delta_{n}$ is exactly $n$. 
This has an immediate consequence in 1 dimension where $-u^{\prime \prime}+V u=E u$ can have at most two solutions.

COROllaRY C.5.7. If $\nu=1, H$ has multiplicity at most 2.

This can be improved.

LemMA C.5.8. Let $\nu=1, V_{-} \in K_{\nu}, V_{+} \in K_{\nu}^{\text {loc }}$. Define

$$
E_{ \pm}=\lim _{R \rightarrow \infty} \inf \left\{(\varphi, H \varphi) /(\varphi, \varphi) \mid \operatorname{supp} \varphi \subset\left\{x \mid x_{<-R}^{>R}\right\}\right\} .
$$

If $E<E_{ \pm}$, then $\mathrm{Hu}=\mathrm{Eu}$ has at most one polynomially bounded solution as $E \rightarrow \pm \infty$.

Remarks. 1. Looking at $E_{ \pm}$is suggested by the general result of Persson [152] (see also Agmon [2] who rediscovered the result) that in general

$$
\text { inf ess } \operatorname{spec}(H)=\lim _{R \rightarrow \infty} \inf \{(\varphi, H \varphi) /(\varphi, \varphi) \mid \operatorname{supp} \varphi \subset\{x|| x \mid>R\}\}
$$

(see Theorem C.8.2).

2. Since the inf is monotone increasing in $R$, the limit exists. It cannot be $-\infty$, but may be $+\infty$.

Proof. We consider the + case. If $E<E_{+}$, then we can find $R$ so that $(\varphi, H \varphi) \geqslant \frac{1}{2}\left(E+E_{+}\right)(\varphi, \varphi)$ if supp $\varphi \subset\{x \mid x>R\}$. Let $W$ be defined by

$$
W(x)= \begin{cases}V(x) & \text { if } x>R, \\ \frac{1}{2}\left(E+E_{+}\right) & \text {if } x<-R, \\ \alpha & \text { if }|x| \leqslant R,\end{cases}
$$

where $\alpha$ will be picked shortly. By (C35) and a small additional argument [53], ess $\operatorname{spec}\left(H_{0}+W\right)=\left[\frac{1}{2}\left(E+E_{+}\right), \infty\right)$. If $\alpha$ is large and positive, then $H_{0}+W$ has no spectrum in $\left(-\infty, \frac{1}{2}\left(E+E_{+}\right)\right]$but as $\alpha \rightarrow-\infty$ more and more eigenvalues exist at lower and lower energies. By continuity, we can find $\alpha$ with $E \in \operatorname{spec}\left(H_{0}+W\right)$. If $u$ is the corresponding eigenfunction, then $u$ obeys $H u=E u$ in $|x|>R$ and $u, u^{\prime}$ decay exponentially at $+\infty$ by Theorem C.3.4. If $\tilde{u}$ is another polynomially bounded solution, $\tilde{u}^{\prime}$ will be polynomially bounded in $L^{2}$ sense so that the Wronskian of $u$ and $\tilde{u}$ is in $L^{1}$ at $\infty$ which implies it is 0 ; i.e. there is exactly one solution polynomially bounded at $+\infty$.

Thus we have

Corollary C.5.9. If $E_{ \pm}$are given by (C39) and if $E<\max \left(E_{+}, E_{-}\right)$, then the spectral multiplicity of $H$ in $(-\infty, E)$ is at most 1 .

REMARK. For the a.c. spectrum, a related result appears in [49].

Open question. Obtain some efficient bounds on multiplicities in the quasione-dimensional case where $V$ is confined in $\nu-1$ dimensions. For example, if we write a point in $R^{\nu}$ as $(x, y)$ with $x \in R, y \in R^{\nu-1}$, we might suppose that $-\frac{1}{2} \Delta_{y}+V(y, x) \equiv H(x)$ on $L^{2}\left(R^{\nu-1}\right)$ has compact resolvent for each $x$. Let $\left\{E_{j}(x)\right\}_{j=1}^{\infty}$ denote the eigenvalues of $H(x)$. Define $E_{j}^{(0)} \equiv \inf _{x} E_{j}(x)$ and $E_{j}^{ \pm}=\lim _{x \rightarrow \pm \infty} E_{j}(x)$. We conjecture: (i) If $\lim _{j \rightarrow \infty} E_{j}^{(0)}=\infty$, then the spectral multiplicity of $H=-\frac{1}{2} \Delta+V$ is finite at any energy, albeit perhaps unbounded as energy goes to infinity. (ii) Explicitly, on $\left(-\infty, E_{j}^{(0)}\right)$, the maximal 
multiplicity is at most $2 j$. (iii) In more refined form, the maximal multiplicity on $(-\infty, E)$ is bounded by $j+k$ if $E<E_{j}^{(0)}$ and $E<E_{k}^{+}$(or $E_{k}^{-}$).

We now want to return to the general properties of eigenfunctions. If one goes through the proof above carefully, one learns that for any fixed $f \in L^{2}$, $f(x) \varphi_{j}(x, \lambda) \in L^{2}$ for all $j$ and a.e. $\lambda$. This would seem to imply that $\varphi_{j} \in L^{\infty}$, but unfortunately, the set of $\lambda$ for which $f \varphi_{j} \notin L^{2}$ could depend on $f$. Nevertheless we conjecture that $\varphi_{j} \in L^{\infty}$ :

Open question. Prove or disprove that for a.e. $\lambda$ (with respect to $\rho$ ), we have that $\varphi_{j}(x, \lambda) \in L^{\infty}$ for all $j$.

We note that it is not hard to show that

$$
\sup _{x}\left[\int_{\Delta} d \rho(\lambda) \sup _{j}\left|\varphi_{j}(x, \lambda)\right|\right]<\infty .
$$

It is often stated (e.g. [23, 54]) that the question just raised has been settled by Maslov [132] who claims to have an example where $\left\{\lambda \mid \varphi_{j} \in L^{\infty}\right\}$ does not exhaust supp $\rho$. We should analyze (following Faris [53]) this example to explain why the question remains open.

EXAMPle (MASLOV [132]). Let $V(x)=V(-x)$ on $(-\infty, \infty)$ where for $x>0$

$$
\begin{array}{rlrl}
V(x) & =x, & & 0 \leqslant x<1, \\
& =\frac{1}{2}(x-1), & & 1 \leqslant x<3, \\
& =\frac{1}{4}(x-3), & & 3 \leqslant x<7, \\
& \vdots & & \\
& =\frac{1}{2^{n}}\left(x-2^{n}+1\right), & 2^{n}-1 \leqslant x<2^{n+1}-1,
\end{array}
$$

Maslov shows that for any $E \in(0,1)$, some solution of $H u=E u$ is (at least logarithmically) unbounded. Let $\mathcal{H}^{ \pm}=\left\{f \in L^{2} \mid f(x)= \pm f(-x)\right\}$. Then $H$ leaves $\mathcal{K}^{ \pm}$invariant and Maslov shows that $\operatorname{spec}\left(H \uparrow \mathcal{K}^{ \pm}\right)=[0, \infty)$. He concludes that the spectral multiplicity of $H$ is 2 so it cannot happen that $\varphi_{j} \in L^{\infty}$ for a.e. $\lambda \in(0,1)$. The error of Maslov is that $\operatorname{spec}\left(H \uparrow \mathcal{F}^{+}\right)=$ $\operatorname{spec}\left(H \uparrow \mathcal{H}^{-}\right)$does not imply that $H$ has multiplicity 2 . In fact, it may well be that $H$ has dense point spectrum in $[0,1]$ in which case, it would of necessity have simple spectrum there!

As a final consequence of the results of this section, we want to demonstrate the fact that if $H$ has infinite point spectrum in some interval (either dense point spectrum as occurs in certain random systems [77] or an accumulation point of discrete spectrum), then the corresponding eigenfunctions must be localized in different regions.

TheOREM C.5.10. Let $V_{+} \in K_{\nu}^{\text {loc }}, V_{-} \in K_{\nu}$. Suppose that a bounded interval $\Delta$ has infinitely many eigenvalues $\left\{\lambda_{j}\right\}_{j=1}^{\infty}$ for $H=-\frac{1}{2} \Delta+V$ with eigenvalues $\varphi_{j}(x)$. Then $L(x) \equiv \sum_{j=1}^{\infty}\left|\varphi_{j}(x)\right|^{2}<\infty$ for each $x$ (and $L$ is polynomially bounded). 
Proof. We will show that function $d E(x, y ; \lambda) / d \rho$ is nonnegative on diagonal and that

$$
L(x) \leqslant \int_{\Delta} d \rho(\lambda) \frac{d E}{d \rho}(x, x ; \lambda) .
$$

For the first assertion, we need only show that the trace class operator $a(\lambda)$ has an integral kernel which is positive on diagonal. This follows from the fact that it is a positive operator with continuous integral kernel.

Next we note that for any fixed $\lambda$, the spectral projection $E(\{\lambda\})$ is exactly the projection onto all eigenvectors with eigenvalue $\lambda$; thus if $\lambda$ is an eigenvalue

$$
\frac{d E}{d \rho}(x, y ; \lambda)=[\rho(\lambda)]^{-1} \sum_{k} \psi_{k}(x) \overline{\psi_{k}(y)},
$$

$\left\{\psi_{k}\right\}$ being a basis for $\operatorname{Ran} E(\{\lambda\})$. Using continuity of the eigenvectors,

$$
\frac{d E}{d \rho}(x, x ; \lambda)=\rho(\lambda)^{-1} \sum_{k}\left|\psi_{k}(x)\right|^{2}
$$

and so (C37) holds.

Open question. The following is somewhat related to unique continuation (see §C9). Is $d E(x, x ; \lambda) / d \rho$ a strictly positive function for a.e. $\lambda$ ?

C6. The local spectral density and its classical limit. At the end of the last section, we showed that $d E(x, x ; \lambda) / d \rho(\lambda) \geqslant 0$. In this section we will discuss the measure on $R^{\nu} \times R$ :

$$
d L(x, \lambda) \equiv \frac{d E(x, x ; \lambda)}{d \rho} d \rho(\lambda) d^{\nu} x .
$$

By very different methods, Lavine [127] introduced this object in a distinct guise and studied its classical limit. Following [127], we call $L$ the local spectral density. Lavine required more local regularity on $V$ but didn't require $H$ to be bounded below. Except for this difference on hypotheses of $V$, we recover Lavine's results here. Lavine introduces $d L$ by the following characterization.

TheOREM C.6.1. Let $V_{+} \in K_{\nu}^{\text {loc }}, V_{-} \in K_{\nu}$. Then, for every bounded Borel function $g$ of compact support in $R$ and every positive $f \in L^{2}\left(R^{\nu}\right)$, $f^{1 / 2}(x) g(H) f^{1 / 2}(x)$ is in trace class and

$$
\operatorname{Tr}\left(f(x)^{1 / 2} g(H) f(x)^{1 / 2}\right)=\int f(x) g(\lambda) d L(x, \lambda) .
$$

Proof. That $f^{1 / 2}(x)|g(H)|^{1 / 2}$ is in $g_{2}$ is proven in Theorem B.9.1 which demonstrates sufficient continuity in $f, g$ to show that (C39) need only be proven for $f \in C_{0}^{\infty}$ and $g$ the characteristic function of an interval $\Delta$. Thus we need only show that

$$
\operatorname{Tr}\left(f(x)^{1 / 2} E(\Delta) f(x)^{1 / 2}\right)=\int_{\lambda \in \Delta} f(x) \frac{d E(x, x ; \lambda)}{d \rho(\lambda)} d \rho(\lambda) d^{\nu} x .
$$

But, since $f^{1 / 2} d E f^{1 / 2} / d \rho$ is a trace class operator with continuous kernel,

$$
\operatorname{Tr}\left(f^{1 / 2} \frac{d E}{d \rho} f^{1 / 2}\right) \equiv \int\left[f(x) \frac{d E(x, x ; \lambda)}{d \rho(\lambda)}\right] d^{\nu} x
$$


(see the proof of Theorem 3.9 of [195]). By definition of $d E / d \rho$,

$$
\operatorname{Tr}\left(f^{1 / 2} E(\Delta) f^{1 / 2}\right) \equiv \int_{\Delta} d \rho(\lambda) \operatorname{Tr}\left(f^{1 / 2} \frac{d E}{d \rho} f^{1 / 2}\right) .
$$

Introducing $H_{\hbar} \equiv-\hbar^{2} \Delta / 2+V(x)$, one can define measures $d L_{\hbar}(x, \lambda)$ so that (C39) holds if $H$ is replaced by $H_{\hbar}$. In some sense, one expects that as $\hbar \rightarrow 0, d L_{\hbar}$ should approach $(2 \pi \hbar)^{-\nu} \delta\left(\frac{1}{2} p^{2}+V-\lambda\right) d^{\nu} p d^{\nu} x$. In fact

TheOREM C.6.2 (LAVINe [127]). Let $V$ be continuous on $R^{\nu}$ and bounded below. Let $f \in C_{0}^{\infty}\left(R^{\nu}\right)$ and $g$ a continuous, bounded Borel function of compact support. Then

$$
\lim _{\hbar \downarrow 0} \hbar^{\nu} \operatorname{Tr}\left(f(x) g\left(H_{\hbar}\right)\right)=\int g\left(p^{2}+V(x)\right) f(x) \frac{d^{\nu} x d^{\nu} p}{(2 \pi)^{\nu}} .
$$

REMARKS. 1. The earliest results of this genre seem to be due to Berezin [24]. See $[42,188,194,201]$ for further discussion.

2. As noted on p. 107 of [194], it suffices that $V$ be locally $L^{1}$ and say, bounded below. Presumably, $V_{-} \in K_{\nu}$ and $V_{+} \in K_{\nu}^{\text {loc }}$ will suffice.

SKeTCH. Let $g(\lambda)=e^{-\alpha \lambda}$. It suffices to prove the result for such $g$ by a limiting argument. Let $d \mu_{0 ; 0,0, t}$ be conditional Brownian motion starting at $b(0)=0$ and conditioned to $b(t)=0$ (see [194]). Let

$$
\begin{aligned}
& F(t)=t^{\nu / 2} \int d^{\nu} x f(x) \int d \mu_{0 ; 0,0, t}(\omega) \exp \left(-\alpha t^{-1} \int_{0}^{t} V(x+\omega(s)) d s\right), \\
& F(0) \equiv(2 \pi)^{-\nu / 2} \int f(x) \exp (-\alpha V(x)) d^{\nu} x .
\end{aligned}
$$

As in the proofs of Theorem 10.1 of [194], (C40) for $g(\lambda)=e^{-\alpha \lambda}$ is just $\lim _{t \downarrow 0} F(t)=F(0)$. The proof is even easier than in [194] where $f$ is not present (so there is a solvable difficulty in controlling the $d^{\nu} x$ integral). By the scaling properties of Brownian motion

$$
F(t)=\int d^{\nu} x f(x) \int d \mu_{0 ; 0,0,0,1}(\omega) \exp \left(-\alpha \int_{0}^{1} V(x+\sqrt{t} \omega(s)) d s\right)
$$

so that $\lim _{t \downarrow 0} F(t)=F(0)$ is immediate from the continuity of paths and of $V$ and the dominated convergence theorem.

(C40) says that $\hbar^{\nu} d L_{\hbar}$ converges weakly to the measure

$$
d \mu_{\mathrm{cl}}(\lambda, x) \equiv \delta\left(\frac{1}{2} p^{2}+V(x)-\lambda\right) d^{\nu} p d^{\nu} x /(2 \pi)^{\nu}
$$

C7. The integrated density of states. Here we consider some general features of an object of considerable use in analyzing Schrödinger operators when $V$ is random, periodic or almost periodic. Fix $\nu$. Let $\mathfrak{X}_{R}$ denote the characteristic function of $\{x|| x \mid<R\}$ and let $\tau_{R}$ be its volume.

Definition. Let $V$ be a potential with $V_{-} \in K_{\nu}, V_{+} \in K_{\nu}^{\text {loc }}$. Let $H=-\frac{1}{2} \Delta$ $+V$ as usual. We say that $H$ has a density of states measure if and only if for all $g \in C_{0}^{\infty}(R)$

$$
\lambda(g)=\lim _{R \rightarrow \infty} \tau_{R}^{-1} \operatorname{Tr}\left(\mathcal{X}_{R} g(H)\right)
$$

exists. 
It is easy to see that if it exists $\lambda$ defines a positive linear functional of $g$ and so there is a Borel measure, $d k$, called the density of states measure, with

$$
\lambda(g)=\int g(\lambda) d k(\lambda)
$$

The quantity

$$
k(\lambda) \equiv \int_{-\infty}^{\lambda-0} d k(\mu)
$$

is called the integrated density of states. $d k$ is precisely the weak limit of the measures $d k_{R}$ given by

$$
d k_{R}(\lambda)=\tau_{R}^{-1} \int_{|x|<R} d L(x, \lambda)
$$

with $d L$ the local spectral density defined in the last section. Note that although the measures $d k_{R}$ are all absolutely continuous with respect to $\rho$, indeed

$$
\frac{d k_{R}(\lambda)}{d \rho}=\tau_{R}^{-1} \int_{|x|<R} \frac{d E}{d \rho}(x, x ; \lambda),
$$

it can happen that $d k$ is not $d \rho$ absolutely continuous. In fact, for suitable classes of random potentials in 1 dimension, one can show that for a.e. $V$ in the support of the distribution of $V$ 's $d k$ exists and is independent of $V$ [21] while for a.e. pair $(V, W)$, the measures $d \rho$ are mutually singular [146]!

Standard weak limit arguments show that

Proposition C.7.1. If $k$ exists and is continuous at $\lambda$, then

$$
k(\lambda)=\lim _{R \rightarrow \infty} \tau_{R}^{-1} \operatorname{Tr}\left(\mathcal{X}_{R} E_{(-\infty, \lambda)}(H)\right) .
$$

We first want to consider several general features of $k$ : (i) upper and lower bounds on $\lambda(g)$, (ii) independence of boundary conditions, (iii) continuity (in the sense of weak convergence of measures) in $V$.

Proposition C.7.2. The density of states exists if and only if for all $t>0$

$$
\mathcal{L}_{V}(t) \equiv \lim _{R \rightarrow \infty} \tau_{R}^{-1} \operatorname{Tr}\left(\mathcal{X}_{R} e^{-t H}\right)
$$

exists.

Proof. By Theorem B.7.1, $\mathfrak{X}_{R} e^{-t H} \mathcal{X}_{R}$ has a continuous integral kernel away from $|x|=R$ or $|y|=R$ and this kernel is bounded by a constant $M_{t}$ independent of $R$. Thus

$$
\mathcal{E}_{V}^{(R)}(t)=\tau_{R}^{-1} \operatorname{Tr}\left(\mathcal{X}_{R} e^{-t H}\right)
$$

is bounded by $M_{t}$ independently of $R$.

Suppose (C41) exists for all $g$ in $C_{0}^{\infty}$. Fix $\lambda>0$. Pick $g_{k}$ in $C_{0}^{\infty}$ with $0 \leqslant g_{k}(\lambda) \leqslant e^{-t \lambda}$ and $g_{k}(\lambda)=e^{-t \lambda}$ for $\lambda \leqslant k$. Then $0 \leqslant e^{-t \lambda}-g_{k}(\lambda) \leqslant$ $e^{-t k / 2} e^{-t \lambda / 2}$ so

$$
0 \leqslant \tau_{R}^{-1} \operatorname{Tr}\left(\mathscr{X}_{R} e^{-t H}\right)-\tau_{R}^{-1} \operatorname{Tr}\left(\mathscr{X}_{R} g_{k}(H)\right) \leqslant M_{t / 2} e^{-t k / 2}
$$

so the limit (C42) exists. 
Conversely, suppose that (C42) exists. Given $g \in C_{0}^{\infty}$, we can by the Stone-Weierstrass theorem, find polynomials $P_{k}(\lambda)$ in $e^{-\lambda}$ so that

$$
\sup _{\lambda \in \operatorname{spec}(H)} e^{+\lambda}\left[g(\lambda)-P_{k}(\lambda)\right] \rightarrow 0 \quad \text { as } k \rightarrow \infty .
$$

We compute, with $F_{k}(\lambda)=e^{\lambda}\left[g(\lambda)-P_{k}(\lambda)\right]$,

$$
\begin{aligned}
& \left|\operatorname{Tr}\left[\mathfrak{X}_{R} g(H) \mathfrak{X}_{R}-\mathcal{X}_{R} P_{k}(H) \mathfrak{X}_{R}\right]\right| \\
& \quad=\operatorname{Tr}\left(\mathfrak{X}_{R} e^{-H / 2} F_{k}(H) e^{-H / 2} \mathfrak{X}_{R}\right) \leqslant\left\|F_{k}\right\|_{\infty} \operatorname{Tr}\left(\mathfrak{X}_{R} e^{-H} \mathfrak{X}_{R}\right)
\end{aligned}
$$

and so obtain the existence of the limit (C41).

TheOREM C.7.3. (a) Let $V_{+} \in K_{\nu}^{\text {loc }}, V_{-} \in K_{\nu}$. Then for any $g \in C_{0}^{\infty}$

$$
\varlimsup_{R \rightarrow \infty} \tau_{R}^{-1}\left|\operatorname{Tr}\left(\chi_{R} g(H)\right)\right|<\infty .
$$

(b) If $V \in K_{\nu}$, then for suitable $g \in C_{0}^{\infty}, g>0$,

$$
\varlimsup_{R \rightarrow \infty} \tau_{R}^{-1} \operatorname{Tr}\left(\mathscr{X}_{R} g(H)\right)>0 .
$$

Proof. (a) follows from $g(H) \leqslant C e^{-H}$ for suitable $C$ and Theorem B.7.1.

(b) follows from $\left(a^{\prime \prime}\right)$ of the same theorem, since the proof of Proposition C.7.2 shows that if $\varlimsup_{i m}=0$ for all $g>0$, then $\mathcal{L}_{\nu}(t)=0$.

If $V_{+} \notin K_{\nu}$, then it can happen that the limit is zero; indeed, if $H$ has compact resolvent, then $k \equiv 0$. Here is an example where the density of states fails to exist.

EXAMPLE 1. Let $\nu=1$ and given $V$ so that $V(x)=V(-x)$ and for $x>0$

$$
\begin{aligned}
V(x)=0 & \text { if } L_{2 n} \leqslant x<L_{2 n+1}, \\
=1 & \text { if } L_{2 n+1} \leqslant x<L_{2 n+2},
\end{aligned}
$$

where $L_{0}=0, L_{n+1}=L_{n}+2^{2^{n}}$. By a simple path integral estimate if $x \in$ $\left(L_{2 n}+\alpha, L_{2 n+1}-\alpha\right),\left|e^{-t H}(x, x)-(2 \pi t)^{-1 / 2}\right| \leqslant C(t) e^{-d(t) \alpha^{2}}$ and if $x \in$ $\left(L_{2 n+1}+\alpha, L_{2 n+2}-\alpha\right),\left|e^{-t H}(x, x)-(2 \pi t)^{-1 / 2} e^{-t}\right| \leqslant C(t)^{-d(t) \alpha^{2}}$. Thus

$$
\lim _{n \rightarrow \infty}\left(2 L_{2 n}\right)^{-1} \operatorname{Tr}\left(X_{L_{2 n}} e^{-t H}\right)=(2 \pi t)^{-1 / 2} e^{-t}
$$

but

$$
\lim _{n \rightarrow \infty}\left(2 L_{2 n+1}\right)^{-1} \operatorname{Tr}\left(\mathcal{X}_{L_{2 n+1}} e^{-t H}\right)=(2 \pi t)^{-1 / 2} \text {. }
$$

In spite of this, the existence of the limit and its value is independent of boundary conditions.

THEOREM C.7.4. (a) Let $V_{+} \in K_{\nu}^{\text {loc }}, V_{-} \in K_{\nu}$. Let $H_{R}^{D}$ be the operator $H_{0, R}^{D}+V$ where $H_{0, R}^{D}$ is the Laplacian on $L^{2}(|x| \leqslant R)$ with zero boundary conditions on $|x|=R$. Then

$$
\lim _{R \rightarrow \infty} \tau_{R}^{-1}\left[\operatorname{Tr}\left(g(H) X_{R}\right)-\operatorname{Tr}\left(g\left(H_{R}^{D}\right)\right)\right]=0 .
$$

(b) Let $\tilde{X}_{R}$ be the characteristic function of the cube centered at zero of side $R / 2$. Let $H_{R}^{P}$ be the corresponding operator with periodic boundary conditions. 
Then

$$
\lim _{R \rightarrow \infty} \tau_{R}^{-1}\left[\operatorname{Tr}\left(g(H) \tilde{X}_{R}\right)-\operatorname{Tr}\left(g\left(H_{R}^{P}\right)\right)\right]=0 .
$$

(c) Let $\nu=1$. Let $H_{R}$ be any sequence of operators on $L^{2}(-R, R)$ which are form extensions of the form $-d^{2} / d x^{2}+V(x)$ on $C_{0}^{\infty}(-R, R)$ with $\inf _{R}\left[\inf \operatorname{spec}\left(H_{R}\right)\right]>-\infty$. Then

$$
\lim _{R \rightarrow \infty}(2 R)^{-1}\left[\operatorname{Tr}\left(g(H) X_{R}\right)-\operatorname{Tr}\left(g\left(H_{R}\right)\right)\right]=0 .
$$

Proof. (a), (b) By the proof of Proposition C.7.2, we need only prove the result for $g(\lambda)=e^{-t \lambda}$. A simple path integral estimate shows that

$$
\left|e^{-t H}(x, x)-e^{-t H_{R}^{*}}(x, x)\right| \leqslant C \exp \left(-d(t)\left[R-|x|_{*}\right]^{2}\right)
$$

where $|x|_{*}=\left(\sum x_{1}^{2}\right)^{1 / 2}$ if $*=D$ and $=\max _{i}\left|x_{i}\right|$ if $*=P$. This immediately yields the result.

(c) As in the proof of Proposition C.7.2, we need only consider the case $g(\lambda)=(\lambda+a)^{-1}$ for all large $a$. But $\left(H_{R}+a\right)^{-1}-\left(H_{R}^{D}+a\right)^{-1}$ has rank at most 4 , so for this $g$

$$
\left|\operatorname{Tr}\left(g\left(H_{R}\right)\right)-\operatorname{Tr}\left(g\left(H_{R}^{D}\right)\right)\right| \leqslant(4 / R)\left[a+\inf \operatorname{spec}\left(H_{R}\right)\right]^{-1} .
$$

If $g$ is taken as the characteristic function of $(-\infty, \lambda)$, then $\operatorname{Tr}\left(g\left(H_{R}^{*}\right)\right)$ is exactly the number of eigenvalues of $H_{R}^{*}$ in that interval. This explains the reason for the name "density of states".

We have the following continuity result:

THEOREM C.7.6. Let $V_{m} \rightarrow V$ in $K_{\nu}$-norm with $V_{m}, V \in K_{\nu}$. Suppose that each $H_{m}$ has a density of states measure $d k_{m}$. Then $H$ has a density of states measure $d k$ and $d k_{m} \rightarrow d k$ weakly.

Proof. By the argument of Proposition C.7.2, it suffices to prove that $\mathcal{L}_{V}^{(R)}(t)$ has a limit for all $t$ and that $\mathcal{E}_{V_{m}}(t) \rightarrow \mathcal{L}_{V}(t)$. This follows if we show that

$$
\lim _{m \rightarrow \infty} \sup _{R}\left|\mathfrak{L}_{V_{m}}^{(R)}(t)-\mathfrak{L}_{V}^{(R)}(t)\right|=0
$$

for all $t$. But since $e^{-t H_{m}}$ has a continuous integral kernel

(C43) follows from Theorem B.10.1.

$$
\begin{aligned}
\left|\mathcal{E}_{V_{m}}^{(R)}(t)-\mathcal{E}_{V}^{(R)}(t)\right| & \leqslant \sup _{k}\left|e^{-t H}(x, x)-e^{-t H_{m}}(x, x)\right| \\
& \leqslant\left\|e^{-t H}-e^{-t H_{m}}\right\|_{1, \infty},
\end{aligned}
$$

REMARK. Local $K_{\nu}$ convergence is not sufficient. For example [19], if $H_{\alpha, \theta} \equiv-d^{2} / d t^{2}+\cos x+\lambda \cos (\alpha x+\theta)$, then for $\lambda, \theta$ fixed $d k_{\alpha}$ is continuous at the irrational values of $\alpha$ but, in general, discontinuous at the rational values!

THEOREM C.7.7. Let $V$ be in $K_{\nu}$ and in the $K_{\nu}$-closure of $C_{0}^{\infty}$. Then the density of states exists and is identical to that for $V=0$, i.e.

$$
d k(\lambda)=d k_{0}(\lambda)=(2 \pi)^{-\nu / 2}\left[\Gamma\left(\frac{1}{2} \nu\right)\right]^{-1} \lambda^{\nu / 2-1} d \lambda
$$


REMARK. The formula for $d k_{0}$ comes from inverting $\varrho_{0}(t)=(2 \pi t)^{-\nu / 2}$.

Proof. By the last theorem, it suffices to prove the result when $V \in C_{0}^{\infty}$. But in that case if $\operatorname{supp} V \subset\{x|| x \mid<R\}$

$$
\left|e^{-t H}(x, x)-e^{-t H_{0}}(x, x)\right| \leqslant C(t) \exp \left(-d(t)\left[|x|^{2}-R^{2}\right]\right)
$$

from which $\mathcal{L}(t)=\mathscr{L}_{0}(t)$ follows.

A slightly more specific argument shows that the same result is true for $N$-body Hamiltonians.

The last result says that for $N$-body potentials, $d k$ is an object of very limited interest. For sufficiently short range potentials, a different object is of interest.

TheOREM C.7.8. If $V \in K_{\nu}$ and $V \in l_{1}\left(L^{2}\right)$, then, for any $g \in C_{0}^{\infty}, g(H)-$ $g\left(H_{0}\right)$ is trace class.

Proof. By an argument of Krein (see e.g. [124]), it suffices to show that $e^{-t H}-e^{-t H_{0}}$ is trace class. Writing

$$
e^{-t H}-e^{-t H_{0}}=\int_{0}^{t} d s e^{-s H} V e^{-(t-s) H_{0}}
$$

it suffices that $e^{-t H / 2} V$ and $V e^{-t H_{0} / 2}$ be trace class. This follows from Theorem B.9.2.

One can further show that $\operatorname{Tr}\left(g(H)-g\left(H_{0}\right)\right)$ is of the form $\int g(\lambda) d \mu(\lambda)$ for a signed measure $d \mu$. The corresponding distribution function $\int_{-\infty}^{\lambda} d \mu(\lambda)$ is called the spectral shift or Krein spectral shift. As discovered by Birman-Krein it is related to the scattering matrix in a simple and beautiful way. For further discussion see $[28,105,124,125]$.

The situations where $k$ is of some interest are random and almost periodic potentials.

Definition. An ergodic class of potentials is a probability measure $d \mu$ on the bounded continuous functions on $R^{\nu}$, so that the map $V(\cdot) \rightarrow V(\cdot+t)$ is a measure-preserving ergodic transformation.

TheOrem C.7.9. (a) (Benderskii-Pastur [21]) If $d \mu$ is an ergodic class of potentials, then for a.e. $V$ in supp $\mu$, the density of states measure exists and $\operatorname{spec}(H)$ is precisely the support of $d k$.

(b) (Avron-Simon [19]) If $V$ is an almost periodic potential, then the density of states measure exists and $\operatorname{spec}(H)$ is precisely the support of $d k$.

REMARKS. 1. For $\nu=1$, a result equivalent to (b) was first proven by Johnson and Moser [106]. [21] proves (a) only if $\nu=1$. For general $\nu$, and additional results, see Pastur [144-147], Fukushima and Nakao [64-66, 140], Kirsch and Martinelli [116], Kotani [121] and Slivnyak [198].

2. Any almost periodic function is naturally associated to an ergodic class of potentials of which it is a member. However (a) does not quite imply (b) since in (a) an assertion is only made for a.e. $V$.

Proof. We prove the first assertion in each part. For the second assertion (i.e. $\operatorname{supp}(d k)=\operatorname{spec}(H)$ ), see [19]. By Proposition C.7.2, it suffices to prove that $\left(1 / \tau_{R}\right) \int_{|x|<R} e^{-t H}(x, x) d x$ has a limit for each fixed rational $t>0$. In case (b), it is not hard (using e.g. Theorem C.10.1) to prove that $f(x)=e^{-t H}(x, x)$ 
is an almost periodic function from which it follows that the limit exists. For (a), we let $g(V)=e^{-t\left(H_{0}+V\right)}(0,0)$ so that $\left(1 / \tau_{R}\right) \int_{|x|<R} g(V(\cdot+x)) d^{\nu} t$ exists by the Birkhoff ergodic theorem.

C8. Allegretto-Piepenbrink theory. We present here some ideas developed in 1974 by Allegretto [13] and Piepenbrink [153, 154] and developed by Moss and Piepenbrink [138] and Allegretto $[14,15]$ who relaxed regularity assumptions on $V$. Some related ideas occur in Albeverio et al. $[10,11]$. Here we will prove Theorem C.8.1 under what are presumably optimal regularity hypotheses; Theorem C.8.2 can probably be improved.

The key notion is that the existence of positive solutions of $H u=E u$ or of solutions positive near infinity is connected to the spectrum of $H$ in $(-\infty, E)$.

TheOREM C.8.1. Let $V_{-} \in K_{\nu}, V_{+} \in K_{\nu}^{\text {loc }}$. Then $H u=E u$ has a nonzero distributional solution which is everywhere nonnegative if and only if inf $\operatorname{spec}(H)$ $\geqslant E$.

Proof. Suppose first that inf $\operatorname{spec}(H) \geqslant E$. Pick $C_{0}^{\infty}$-functions $f_{n}$ supported in $\{x|n<| x \mid<2 n\}$ so that $f_{n} \geqslant 0$ and $f_{n} \neq 0$. Let

$$
u_{n}(x)=c_{n}\left(H-E+n^{-1}\right)^{-1} f_{n}
$$

where $c_{n}$ is chosen so that $u_{n}(0)=1$. Since $u_{n}$ is everywhere positive (by the proof of Lemma B.7.7), $c_{n}$ can be chosen to obtain the required normalization. Clearly, $u_{n}$ obeys $H u_{n}=\left(E-n^{-1}\right) u_{n}$ for the region $|x|<n$. Thus, by Harnack's inequality, for any $R$ we can find $C_{R}$ with

$$
C_{R}^{-1} \leqslant u_{n}(x) \leqslant C_{R} \text { if }|x|<R .
$$

By passing to a subsequence, we can be sure that $u_{n}$ has a limit point in weak ${ }^{*} L_{\text {loc }}^{\infty}$ sense so that $\left(u_{n}, \varphi\right) \rightarrow(u, \varphi)$ for all $\varphi \in L^{1}$ with supp $\varphi$ bounded. It is easy to see that $u$ is a distributional solution of $H u=E u$ and that $C_{R}^{-1} \leqslant u(x)$ $\leqslant C_{R}$ for $|x|<R$, so that $u$ is nonnegative and not identically zero.

Conversely, suppose $H u=E u$ has a nonzero, nonnegative solution. By Harnack's inequality, $u$ is strictly positive and by Theorem C.2.2 $u^{-1} \nabla u \equiv g$ is in $L_{\text {loc }}^{2}$. We will prove that for $\varphi \in C_{0}^{\infty}$

$$
(\varphi,(H-E) \varphi)=\frac{1}{2}\|\nabla \varphi-g \varphi\|_{2}^{2}
$$

which implies that $H-E \geqslant 0$. We first prove (C44) assuming $u$ is $C^{\infty}$. Then by a direct calculation

$$
\frac{1}{2} u^{-1} \nabla u^{2} \nabla u^{-1}=\frac{1}{2}\left[\Delta-u^{-1}(\Delta u)\right]=-(H-E)
$$

so

$$
(\varphi,(H-E) \varphi)=\frac{1}{2}\left\|u \nabla\left(u^{-1} \varphi\right)\right\|^{2}=\frac{1}{2}\left\|\nabla \varphi-\left(u^{-1} \nabla u\right) \varphi\right\|^{2}
$$

proving (C44) in that case. Given general $u, V$, we know that $u$ is continuous and locally bounded away from zero. Let $u_{\delta}$ be $u$ convoluted with an approximate identity $j_{\delta}$. Let $V_{\delta} \equiv u_{\delta}^{-1}\left[\frac{1}{2} \nabla u_{\delta}\right]+E$ so by the above,

$$
\left(\varphi,\left(H_{0}+V_{\delta}-E\right) \varphi\right)=\frac{1}{2}\left\|\nabla \varphi-g_{\delta}\right\|_{2}^{2} \text {. }
$$


But since $u_{\delta} \rightarrow u$ locally uniformly ( $u$ is continuous) and $\nabla u_{\delta} \rightarrow \nabla u$ in $L_{\text {loc }}^{2}$ and $\Delta u_{\delta} \rightarrow \Delta u$ in $L_{\text {loc }}^{1}$, we have that $g_{\delta} \rightarrow g$ in $L_{\text {loc }}^{2}$ and $V_{\delta} \rightarrow V$ in $L_{\text {loc }}^{1}$ proving (C44) in general.

REMARK. The above proof actually shows that for $\varphi \in C_{0}^{\infty},(\varphi,(H-E) \varphi)$ $>0$ (for if $\nabla \varphi-g \varphi=0$ then $\varphi=c u \notin C_{0}^{\infty}$ ) and, in fact, $\operatorname{ker}(H-E$ ) is either empty or multiples of $u$ depending on whether $u \in L^{2}$.

Of course, if $E<\inf \operatorname{spec}(H)$, then $u$ cannot be polynomially bounded. Unfortunately, the converse is false!

EXAMPLE $(\nu=1)$. Let $u$ be a positive $C^{\infty}$-function on $(-\infty, \infty)$ which is equal to $\exp \left(|x|^{1 / 2}\right)$ on $[1, \infty)$ and to $\exp \left(-|x|^{1 / 2}\right)$ on $(-\infty,-1]$. Let $V(x)$ $=\frac{1}{2} u^{\prime \prime} / u$. Then $V$ is $C^{\infty}$ and $V(x)=O\left(x^{-1}\right)$ at infinity. Since $H u=0$ has a positive solution, inf $\operatorname{spec}(H)>0$ but since $u$ is bounded by any exponential, by Remark 3 following Theorem C.4.1, $0 \in \operatorname{spec}(H)$ so $0=\inf \operatorname{spec}(H)$. $u$ is clearly not polynomially bounded at $+\infty$, but no other solution, $v$, can be polynomially bounded at $-\infty$ (for the Wronskian of $u$ and $v$ would be $L^{2}$ at $-\infty$ which is impossible). This illustrates that $H u=\inf \operatorname{spec}(H) u$ may have no polynomially bounded solution, let alone a positive one.

Theorem C.8.2. Let $V_{+} \in K_{\nu, \text { loc }}^{(1)}, V_{-} \in K_{\nu}^{(1)}$. Fix E. Then the following are equivalent:

(a) For some $R,(\varphi, H \varphi)>E(\varphi, \varphi)$ for all $\varphi \in C_{0}^{\infty}$ with supp $\varphi \subset(x|| x \mid$ $>R$ ).

(b) For some $R, H u=E u$ has a nonzero distributional solution on $\{x|| x \mid>R\}$ which is nonnegative there.

(c) The spectral projection for the interval $(-\infty, E]$ is finite dimensional.

For general $V$ with $V_{+} \in K_{\nu}^{\text {loc }}$ and $V_{-} \in K_{\nu}$, (a) is equivalent to (b) and both are implied by (c). Moreover (a), (b) implies inf ess $\operatorname{spec}(H) \geqslant E$.

REMARK. The equivalence (a) $\Rightarrow$ (c) is clearly related to the result of Persson [152] and Agmon [2] which appears as (C35). Indeed, the last assertions in the theorem are precisely (C35).

Proof. (a) $\Rightarrow$ (b) (a) says that if $H_{R}$ is the operator

$$
H_{0}+V \text { on } L^{2}(\{x|| x \mid>R\})
$$

with Dirichlet conditions on $|x|=R$, then $\inf \operatorname{spec}\left(H_{R}\right) \geqslant E$, so $u$ can be constructed as in the last theorem.

(b) $\Rightarrow$ (a) (C44) still holds for $\varphi$ supported in $\{x|| x \mid>R\}$.

(c) $\Rightarrow$ (a) If (a) is false, we can inductively pick $r_{1}<r_{2}<\cdots$ and $\varphi_{n}$ in $C_{0}^{\infty}$ with supp $\varphi_{n} \subset\left\{x\left|r_{n}<\right| x \mid<r_{n+1}\right\}$ with $\left(\varphi_{n}, H \varphi_{n}\right) \leqslant E\left(\varphi_{n}, \varphi_{n}\right)$. Obviously, for $n \neq m,\left(\varphi_{n}, H \varphi_{m}\right)=0$. Thus, if $\Psi$ is any finite linear combination of the $\varphi_{J}$, then $(\Psi, H \Psi) \leqslant E(\Psi, \Psi)$. It follows (see Theorem XIII.1 of [162]) that the spectral projection for the interval $(-\infty, E]$ has infinite dimension.

(a) $\Rightarrow$ [inf ess $\operatorname{spec}(H) \geqslant E$ ] (following ideas of Sigal [177]). Pick $j_{1}, j_{2}$ with $j_{1}$ in $C_{0}^{\infty}$ and $j_{2}$ in $C^{\infty}$ with $j_{2}(x)=0$ if $|x|<R$, so that $j_{1}^{2}+j_{2}^{2}=1$. Now $\left[j_{1},\left[j_{1}, H\right]\right]+\left[j_{2},\left[j_{2}, H\right]\right]=-\left(\nabla j_{1}\right)^{2}-\left(\nabla j_{2}\right)^{2}$ so since $\left(j_{1}^{2}+j_{2}^{2}\right) H=$ $H\left(j_{1}^{2}+j_{2}^{2}\right)=H$, we have that

$$
H=j_{1} H j_{1}+j_{2} H j_{2}-\frac{1}{2}\left(\nabla j_{1}\right)^{2}-\frac{1}{2}\left(\nabla j_{2}\right)^{2} .
$$


Now, by (a), $j_{2} H j_{2} \geqslant E j_{2}^{2}$. Moreover, $j_{1} H j_{1}=j_{1} \tilde{H} j_{1}$ where $\tilde{V}$ is any function with $V(x)=\tilde{V}(x)$ for $x$ in supp $j_{1}$. In particular, we can pick $\tilde{V}$ so that $\tilde{H}$ has compact resolvent. If we do that $\tilde{H} \geqslant E+F$ where $F$ is a finite rank operator so

$$
j_{1} H j_{1}+j_{2} H j_{2} \geqslant E+j_{1} F j_{1}
$$

which implies that inf ess $\operatorname{spec}\left(j_{1} H j_{1}+j_{2} H j_{2}\right) \geqslant E$. But $-\frac{1}{2}\left(\nabla j_{1}\right)^{2}-\frac{1}{2}\left(\nabla j_{2}\right)^{2}$ is $H$-compact so ess $\operatorname{spec}(H)=\operatorname{ess} \operatorname{spec}\left(j_{1} H j_{1}+j_{2} H j_{2}\right)$ (see Theorem XIII.14 of [162]).

(b) $\Rightarrow$ (c) if $V \in K_{\nu}^{(1), l o c}$. Without loss, suppose $E=0$. Pick $j_{1}, j_{2}$ with $j_{1} \in$ $C_{0}^{\infty}, 1-j_{2} \in C_{0}^{\infty}$ and $j_{2}(x)=0$ if $|x|<R+\frac{1}{2}, j_{1}(x)=0$ if $|x| \geqslant R+1$ and $j_{1}^{2}+j_{2}^{2}=1$. Let $w=j_{1}^{2}+j_{2}^{2} u$ which is $C^{1}$ since $V \in K_{\nu}^{(1)}$ and let $\tilde{V}=$ $(w)^{-1}\left[\frac{1}{2} \Delta w\right]$ so $V=\tilde{V}$ if $|x| \geqslant R=1$. Moreover $\tilde{V}$ is easily seen to be $\left[u j_{2}^{2} w^{-1}\right] V$ plus a continuous function and so it is in $K_{\nu}$. Pick $\varphi \in C_{0}^{\infty}$ with $\partial \varphi / \partial n=0$ on $|x|=R+1$. Then

$$
\begin{aligned}
(\varphi,(-\Delta+V) \varphi) & =(\varphi,(-\Delta+\tilde{V}) \varphi)+\int(V-\tilde{V})|\varphi|^{2} d^{\nu} x \\
& =\int\left|\nabla \varphi-w^{-1} \varphi \nabla w\right|^{2}+\int(V-\tilde{V})|\varphi|^{2} d^{\nu} x \\
& \equiv a(\varphi)+b(\varphi)
\end{aligned}
$$

where

$$
\begin{aligned}
& a(\varphi)=\int_{|x|>R+1}\left|\nabla \varphi-w^{-1} \varphi \nabla w\right|^{2} d^{\nu} x, \\
& b(\varphi)=\int_{|x|<R+1} \bar{\varphi}[-\Delta+V] \varphi+\int_{|x|=R+1} d n|\varphi|^{2} \sigma
\end{aligned}
$$

with $\sigma=u \partial u^{-1} / \partial n$ is bounded. This uses an elementary integration by parts. By a limiting argument, this equality holds for any $\varphi$. Let $A, B$ be the operators on $L^{2}(|x| \geqslant R+1)$ and $L^{2}(|x| \leqslant R+1)$ with forms $a, b$. Then $H \geqslant A \oplus B$ (one does not have equality because $A \oplus B$ has a larger form domain; see $\S$ XIII.15 of [162]). Since $\sigma$ is bounded and $V \in K_{\nu}$, it is easy to see that $B$ has compact resolvent. Obviously, $A \geqslant 0$ and $\operatorname{ker}(A)$ is either zero or one dimensional depending on whether $w \in L^{2}$ or not. Thus $A \oplus B$ has a finite rank spectral projection for $(-\infty, 0]$ and so therefore does $H$.

Open question. Does (a) $\Rightarrow$ (c) for any $V$ with $V_{+} \in K_{\nu}^{\text {loc }}, V_{-} \in K_{\nu}$ ?

The answer should be yes.

Corollary C.8.3. Let $V, W$ have $W_{+}, V_{+} \in K_{\nu, l o c}^{(1)} V_{-}, W_{-} \in K_{\nu}^{(1)}$ with $\operatorname{supp}[V-W]$ bounded. Then, for any $E$, the dimension of the spectral projection for the integral $(-\infty, E]$ for $-\frac{1}{2} \Delta+V$ is finite if and only if the same is true for $-\frac{1}{2} \Delta+W$.

Proof. Solutions of $-\frac{1}{2} \Delta u+V u=E u$ outside a large sphere are the same as solutions of $-\frac{1}{2} \Delta u+W u=E u$ there. Use (b) $\Leftrightarrow$ (c) in Theorem C.8.2.

REMARK. The proof of (a) $\Rightarrow$ [inf ess $\operatorname{spec}(H) \geqslant E$ ] above shows that if the corollary could be proved assuming $K_{\nu}$ whenever $K_{\nu}^{(1)}$ appears, then one would know that $(\mathrm{a}) \Rightarrow(\mathrm{c})$ in general. This corollary is due to Piepenbrink [153]. 


\section{C9. Unique continuation.}

Definition. A partial differential operator, $P$, is said to have the unique continuation property (u.c.p.) if and only if every distributional solution $u$ of $P u=0$ in an open connected set, $\Omega$, which vanishes near some $x_{0} \in \Omega$ is identically zero.

The following result is "classical".

THEOREM C.9.1. Let $V$ be a potential which is bounded on compact subsets of $R^{\nu} \backslash \Gamma$ where $\Gamma$ is a closed set of measure zero. Then $-\frac{1}{2} \Delta+V-E$ has the u.c.p.

For $\nu=2$, this is a result of Carleman [33] and for general $\nu$ of Müller [139]. For further discussions and history, see Hörmander's book [99] or Reed and Simon, Vol. IV [162]. The latter uses Carleman's approach together with a bound of Heinz [79]. The following elementary estimates can shorten and replace the Heinz estimates. These estimates are taken from a paper of Hörmander [98] whose proof we follow:

LEMMA C.9.2. For any real $\alpha, \lambda$ and any $u \in C_{0}^{\infty}\left(R^{\nu} \backslash\{0\}\right)$

$$
\left\|r^{\alpha+1}(\Delta+\lambda) u\right\|_{2}^{2} \geqslant 4 \alpha \lambda\left\|r^{\alpha} u\right\|_{2}^{2}
$$

Proof. Let $C=r^{\alpha+1}(\Delta+\lambda) r^{-\alpha+1}$, so

$$
C=r\left(\nabla-\alpha r^{-1}\right)^{2} r+\lambda r^{2}=L_{1}+L_{2}
$$

where

$$
L_{1}=L_{1}^{*}=r \nabla^{2} r+\alpha^{2}+\lambda r^{2}, \quad L_{2}=-L_{2}^{*}=-\alpha[r \nabla+\nabla r] .
$$

Since $L_{2}$ is the generator of dilations $\left[L_{2}, L_{1}\right]=\left[L_{2}, \lambda r^{2}\right]$. Thus

$$
C^{*} C=\left(L_{1}-L_{2}\right)\left(L_{1}+L_{2}\right) \geqslant\left[L_{1}, L_{2}\right]=4 \alpha \lambda r^{2} \text {. }
$$

Let $v=r^{\alpha-1} u$ and note that (C45) says that $\|C v\|_{2}^{2} \geqslant 4 \alpha \lambda\|r v\|_{2}^{2}$.

Theorem C.9.1 follows from the lemma and the Carleman argument found on p. 243 of [162].

This leaves the question of unbounded $V$. The following is a natural conjecture.

Open question. Prove that for any $V$ in $K_{\nu}^{\text {loc }}, H$ has the u.c.p. There are so far some disappointing results on this problem. The first are due to Schechter and Simon [173] who prove

THEOREM C.9.3. If $V^{2}$ is locally $H_{0}$-form bounded, in particular if $V^{2}$ is in $K_{\nu}^{\text {loc }}$, then $H=H_{0}+V$ has the u.c.p.

Schechter and Simon [173] also have some results requiring $L^{p}$-properties of $V$. These have been improved by Saut and Scheurer [169] and by Amrein, Berthier and Georgescu [16, 25, 26, 71] who prove

THEOREM C.9.4. If $V \in L_{\mathrm{loc}}^{p}, p>2 \nu / 3$, then $H_{0}+V$ has the u.c.p. 


\section{REFERENCES}

1. S. Agmon, Spectral properties of Schrödinger operators and scattering theory, Ann. Scuola Norm Sup. Pisa II 2 (1979), 151-218.

2. , On exponential decay of solutions of second order elliptic equations in unbounded domains, Proc. Conf. A. Pleijel.

3.

4. Lectures on exponential decay of solutions of second order elliptic equations. Bounds on eigenfunctions of $\mathrm{N}$-body Schrödinger operators, Mathematical Notes, Princeton Univ. Press, Princeton, N.J., 1982.

5. J. Aguilar and J. Combes, A class of analytic perturbations for one-body Schrödinger Hamiltonians, Comm. Math. Phys. 22 (1971), 269-279.

6. R. Ahlrichs, Asymptotic behavior of atomic bound state wave functions, J. Math. Phys. 14 (1973), 1860-1863.

7. R. Ahlrichs, M. Hoffman-Ostenhof and T. Hoffman-Ostenhof, Bounds on the long-range behavior of atomic wave functions, J. Chem. Phys. 68 (1978), 1402-1410.

8. R. Ahlrichs, M. Hoffman-Ostenhof, T. Hoffman-Ostenhof and J. Morgan, Bounds on the decay of electron densities with screening, Phys. Rev. A 23 (1981), 2106-2116.

9. M. Aizenman and B. Simon, Brownian motion and Harnack's inequality for Schrödinger operators, Comm. Pure Appl. Math. 35 (1982), 209-271.

10. S. Albeverio, R. Hoegh-Krohn and L. Streit, Energy forms, Hamiltonians and distorted Brownian paths, J. Math. Phys. 18 (1977), 907-917.

11. _ Regularization of Hamiltonians and processes, J. Math. Phys. 21 (1980), 1636-1642.

12. V. de Alfaro and T. Regge, Potential scattering, North-Holland, Amsterdam, 1965.

13. W. Allegretto, On the equivalence of two types of oscillation for elliptic operators, Pacific J. Math. 55 (1974), 319-328.

14. Spectral estimates and oscillation of singular differential operators, Proc. Amer. Math. Soc. 73 (1979), 51.

15. _ Positive solutions and spectral properties of second order elliptic operators, Pacific J. Math. 92 (1981), 15-25.

16. W. Amrein, A. Berthier and V. Georgescu, $L^{p}$ inequalities for the Laplacian and unique continuation, Ann. Inst. Fourier (Grenoble) 31 (1981), 153-168.

17. J. Avron and I. Herbst, Spectral and scattering theory of Schrödinger operators related to the Stark effect, Comm. Math. Phys. 52 (1977), 239-254.

18. J. Avron and B. Simon, Singular continuous spectrum for a class of almost periodic Jacobi matrices, Bull. Amer. Math. Soc. (N.S.) 6 (1982), 81-86.

19. _ Almost periodic Schrödinger operators. II, The density of states (preprint)

20. N. Bazley and D. Fox, Bounds for eigenfunctions of one-electron molecular systems, Internat. J. Quant. Chem. 3 (1969), 581-586.

21. M. Benderskii and L. Pastur, On the spectrum of the one dimensional Schrödinger equation with a random potential, Mat. Sb. 82 (1970), 245-256.

22. J. Berezanskii, On expansion according to eigenfunctions of general self-adjoint differential operators, Dokl. Akad. Nauk SSSR 108 (1956), 379-382.

23. __ Expansions in eigenfunctions of selfadjoint operators, Transl. Math. Mono., vol. 17, Amer. Math. Soc., Providence, R.I., 1968.

24. F. Berezin, Wick and anti-Wick symbols, Math. USSR Sb. 15 (1971), 577-606.

25. A. M. Berthier, Sur le spectre poncture de l'operateur de Schrödinger, C. R. Acad. Sci. Paris Sér. A 290 (1980), 393-395.

26. appear). , On the point spectrum of Schrödinger operators, Ann. Sci. École Norm. Sup. (to

27. A. Berthier and B. Gaveau, Critère de convergence des fonctionelles de Kac et applications en mechanique et en géométrie, J. Funct. Anal. 29 (1978), 416-424.

28. M. Birman and M. Krein, On the theory of wave operators and scattering operators, Dokl. Akad. Nauk SSSR 144 (1962), 475-478.

29. H. Brascamp and E. H. Lieb, Best constants in Young's inequality, its converse and its generalization to more than three functions, Adv. in Math. 20 (1976), 151-173.

30. H. Brascamp, E. Lieb and J. Luttinger, A general rearrangement inequality for multiple integrals, J. Funct. Anal. 17 (1974), 227-237. 
31. H. Brezis and T. Kato, Remarks on the Schrödinger operator with singular complex potentials, J. Math. Pures Appl. 58 (1979), 137-151.

32. F. Browder, Eigenfunction expansions for formally self-adjoint partial differential operators. I, II, Proc. Nat. Acad. Sci. USA 42 (1956), 769-771; 870-872.

33. T. Carleman, Sur un problème d'unicité pour les systèmes d'équations aux derivés partielles à deux variables indépendantes, Ark. Mat. 26B (1939), 1-9.

34. R. Carmona, Regularity properties of Schrödinger and Dirichlet semigroups, J. Funct. Anal. 17 (1974), 227-237.

35. Pointwise bounds for Schrödinger eigenstates, Comm. Math. Phys. 62 (1978), 97-106.

36. ___ Exponential localization in one dimensional disordered systems, Duke Math. J. (to appear).

37. R. Carmona and B. Simon, Pointwise bounds on eigenfunctions and wave packets in N-body quantum systems. V. Lower bounds and path integrals, Comm. Math. Phys. 80 (1981), 59-98.

38. K. Chung and S. Varadhan, Kac functional and Schrödinger equation, Studia Math. 68 (1980), 249-260.

39. K. Chung and K. Rao, Sur la théorie du potentiel avec la fonctionelle de Feynman-Kac, C. R. Acad. Sci. Paris Sér. A 290 (1980), 629-631.

40. J. Combes, Time dependent approach to multichannel scattering, Nuovo Cimento A 64 (1969), 111-144.

41. J. M. Combes, M. Hoffman-Ostenhof and T. Hoffman-Ostenhof, Asymptotics of atomic ground states: the relation between the ground states of helium and the ground state of $\mathrm{He}^{+}, \mathrm{J}$. Math. Phys. 22 (1981), 1299-1305.

42. J. Combes, R. Schrader and R. Seiler, Classical bounds and limits for energy distributions of Hamiltonian operators in electromagnetic fields, Ann. Physics 111 (1978), 1-18.

43. J. Combes and L. Thomas, Asymptotic behavior of eigenfunctions for multi-particle Schrödinger operators, Comm. Math. Phys. 34 (1973), 251-270.

44. M. Cwickel, Weak type estimates and the number of bound states of Schrödinger operators, Ann. of Math. (2) 106 (1977), 93-102.

45. E. B. Davies, Properties of Green's functions of some Schrödinger operators, J. London Math. Soc. 7 (1973), 473-491.

46. , Eigenfunction expansions for singular Schrödinger operators, Arch. Rational Mech. Anal. 63 (1977), 261.

47. Some norm bounds and quadratic form inequalities for Schrödinger operators, J. Operator Theory (to appear).

48.

49. E. Davies and B. Simon, Scattering theory for systems with differing asymptotics on the left and right, Comm. Math. Phys. 63 (1978), 277-301.

50. P. Deift, W. Hunziker, B. Simon and E. Vock, Pointwise bounds on eigenfunctions and wave packets in N-body quantum systems. IV, Comm. Math. Phys. 64 (1978), 1-34.

51. M. Eastham, The spectral theory of periodic differential equations, Scottish Academic Press, 1973.

52. J. P. Eckmann, Hypercontractivity for anharmonic oscillators, J. Funct. Anal. 16 (1974), $388-404$.

53. V. Enss, A note on Hunziker's theorem, Comm. Math. Phys. 52 (1977), 233-238.

54. W. Faris, Perturbations and non-normalizable eigenvectors, Helv. Phys. Acta 44 (1971), 936.

55. __, Quadratic forms and essential self-adjointness, Helv. Phys. Acta 45 (1972), 10741088.

56. __ Essential self-adjointness of operators in ordered Hilbert space, Comm. Math. Phys. 30 (1973), 23-34.

57.

58. W. Faris and R. Lavine, Commutators and self-adjointness of Hamiltonian operators, Comm. Math. Phys. 35 (1974), 39-48.

59. H. Federer, Geometric measure theory,Springer-Verlag, Berlin and New York, 1969.

60. R. Froese, I. Herbst, M. Hoffman-Ostenhof and T. Hoffman-Ostenhof, $L^{2}$ exponential lower bounds to solutions of the Schrödinger equation, Comm. Math. Phys. (to appear). 
61. D. Fujiwara, A construction of the fundamental solution for the Schrödinger equation, J. Analyse Math. 35 (1979), 41-96.

62. On a nature of convergence of some Feynman path integrals. I, Proc. Japan Acad. Ser. A Math. Sci. 55 (1979), 195-200.

63. , Remarks on convergence of Feynman path integrals, Duke Math. J. 47 (1980), $559-600$

64. M. Fukushima, On the spectral distribution of a disordered system and the range of a random walk, Osaka J. Math. 11 (1974), 73-85.

65. , On the asymptotics of Schrödinger operators with random potentials, Bielefeld, 1980 (preprint).

66. M. Fukushima and S. Nakao, On spectra of the Schrödinger operator with a white noise potential, Z. Wahrsch. Verw. Gebiete 37 (1977), 267-274.

67. L. Garding, Eigenfunction expansions connected with elliptic differential operators, Tolfte Skend. Mat. Lund, 1953, pp. 44-45.

68. B. Gaveau and E. Mazet, Divergence des fonctionelles de Kac et diffusion quantique, C. R. Acad. Sci. Paris Sér. A 291 (1980), 559-562.

69. I. Gel'fand, Expansion in series of eigenfunctions of an equation with periodic coefficients, Dokl. Akad. Nauk SSSR 73 (1950), 1117-1120.

70. I. Gel'fand and A. Kostjucenko, Expansion in eigenfunctions of differential and other operators, Dokl. Akad. Nauk SSSR 103 (1955), 349-352.

71. V. Georgescu, On the unique continuation property for Schrödinger Hamiltonians, Helv. Phys. Acta 52 (1979), 655-670.

72. D. Gilvarg and N. Trudinger, Elliptic partial differential equations of second order, SpringerVerlag, Berlin and New York, 1977.

73. J. Glimm, Boson fields with nonlinear self-interaction in two dimensions, Comm. Math. Phys. 8 (1968), 12-25.

74. J. Glimm and A. Jaffe, $A \lambda \phi^{4}$ quantum field theory without cutoffs. I, Phys. Rev. 176 (1968), 1945-1951.

75. The $\lambda\left(\phi^{4}\right)_{2}$ quantum field theory without cutoffs. II. The field operators and the approximate vacuum, Ann. of Math. (2) 91 (1970), 362-401.

76. __ Quantum physics, Springer-Verlag, Berlin and New York, 1981.

77. Ya. Goldshtein, S. Molchanov and L. Pastur, A pure point spectrum of the stochastic one-dimensional Schrödinger operator, Funkcional Anal. i Priložen. 11 (1977), 1.

78. L. Gross, Logarithmic Sobolev inequalities, Amer. J. Math. 97 (1976), 1061-1083.

79. E. Heinz, Über die Eindautigkeit beim Cauchyschen Anfabgswertproblem einer elliptischen differentialgleichung zweiter Ordnung, Nach. Akad. Wiss. Göttingen Math.-Phys. K1. II (1955), $1-12$.

80. I. Herbst, Dilation analyticity in constant electric field. The two-body problem, Comm. Math. Phys. 64 (1979), 279.

81. I. Herbst and J. Howland, The Stark ladder and other one-dimensional external field problems, Comm. Math. Phys. 80 (1981), 23.

82. I. Herbst and B. Simon, Dilation analyticity in constant electric field. II. The $\mathrm{N}$-body problem, Borel summability, Comm. Math. Phys. 80 (1981), 181-216.

83. I. Herbst and A. Sloan, Perturbations of translation invariant positivity preserving semigroups in $L^{2}(R)$, Trans. Amer. Math. Soc. 236 (1978), 325-360.

84. H. Hess, R. Schrader and D. Uhlenbrock, Domination of semigroups and generalization of Kato's inequality, Duke Math. J. 44 (1977), 893-904.

85. R. Hoegh-Krohn, $A$ general class of quantum field theories without cutoffs in two space-time dimensions, Comm. Math. Phys. 21 (1971), 244-255.

86. M. Hoffman-Ostenhof and T. Hoffman-Ostenhof, Schrödinger inequalities and asymptotic behavior of the electron density of atoms and molecules, Phys. Rev. A 16 (1977), 1782-1785.

87. M. Hoffman-Ostenhof, T. Hoffman-Ostenhof and B. Simon, On the nodal structure of atomic eigenfunctions, J. Phys. A 13 (1980), 1131-1133.

88. Brownian motion and a consequence of Harnack's inequality: nodes of quantum wave functions, Proc. Amer. Math. Soc. 80 (1980), 301-305.

89. M. Hoffman-Ostenhof and R. Seiler, Cusp conditions for eigenfunctions of $\mathrm{N}$-electron systems, Phys. Rev. A 23 (1981), 21-23. 
90. T. Hoffman-Ostenhof, A comparison theorem for differential inequalities with applications in quantum mechanics, J. Phys. A 13 (1980), 417-424.

91. Lower and upper bounds to the decay of the ground state one-electron density of helium-like systems, J. Phys. A 12 (1979), 1181-1187.

92. (1980), 140-142.

93. T. Hoffman-Ostenhof and M. Hoffman-Ostenhof, Bounds to expectation values and exponentially decreasing upper bounds to the one electron density of atoms, J. Phys. B 11 (1978), 17-24.

94. 59 (1976), 373-374. Exponentially decreasing upper bounds to the electron density of atoms, Phys. Lett. A

95. T. Hoffman-Ostenhof, M. Hoffman-Ostenhof and R. Ahlrichs, Schrödinger inequalities and asymptotic behavior of many electron densities, Phys. Rev. A 18 (1978), 328-334.

96. J. Hooton, Dirichlet forms associated with hypercontractive semigroups, Trans. Amer. Math. Soc. 253 (1979), 237-256.

97. __ Dirichlet semigroups on bounded domains, Louisiana State Univ. (preprint).

98. L. Hörmander, Seminar Schwarz, 1981.

99. Linear partial differential operators, Springer-Verlag, Berlin and New York, 1964.

100. W. Hunziker, Space-time behavior of Schrödinger wave functions, J. Math. Phys. 7 (1966), $300-304$.

101. T. Ikebe, Eigenfunction expansions associated with the Schrödinger operators and their applications to scattering theory, Arch. Rational Mech. Anal. 5 (1960), 1-34; Erratum: J. Fac. Sci. Tokyo Univ. 17 (1970), 355-361.

102. Spectral representation for Schrödinger operators with long-range potentials. I, II,

J. Funct. Anal. 20 (1975), 158-177; Publ. Res. Inst. Math. Sci. 11 (1976), 551-558.

103. T. Ikebe and H. Isozaki, Completeness of modified wave operators for long range potentials, Publ. Res. Inst. Math. Sci. 15 (1979), 679-718.

104. H. Isozaki, On the long-range stationary wave operator, Publ. Res. Inst. Math. Sci. 13 (1977), 589-626.

105. A. Jensen and T. Kato, Asymptotic behavior of the scattering phase for exterior domains, Comm. Partial Differential Equations 3 (1978), 1165-1195.

106. R. Johnson and J. Moser, The rotation number for almost periodic potentials, ETH (preprint).

107. G. Kac, Expansion in characteristic functions of self-adjoint operators, Dokl. Akad. Nauk SSSR 119 (1958), 19-22.

108. H. Kalf, U. Schminzke, J. Walter and R. Wüst, On the spectral theory of Schrödinger and

Dirac operators with strongly singular potentials, Lecture Notes in Math., vol. 448, Springer-Verlag, Berlin and New York, 1975.

109. B. Karlerson, Self-adjointness of Schrödinger operators, Institut Mittag-Leffler Rep. no. 6, 1976.

110. T. Kato, On the eigenfunctions of many particle systems in quantum mechanics, Comm. Pure Appl. Math. 10 (1957), 151-171.

111. Wave operators and similarity for some non-selfadjoint operators, Math. Ann. 162 (1966), 258-279.

112. __ Schrödinger operators with singular potentials, Israel J. Math. 13 (1973), 135-148.

113. Remarks on Schrödinger operators with vector potentials, Integral Equations Operator Theory 1 (1978), 103-113.

114. __ Perturbation theory for linear operators, Springer-Verlag, Berlin and New York, 1966.

115. R. Khas'minskii, On positive solutions of the equation $U u+V u=0$, Theory Probab. Appl. 4 (1959), 309-318.

116. W. Kirsch and F. Martinelli, On the density of states of Schrödinger operators with random potential, J. Phys. A. (to appear).

117. H. Kitada, Scattering theory for Schrödinger operators with long range potential. I, II, J. Math. Soc. Japan 29 (1977), 655-691; 30 (1978), 603-632.

118. , On a construction of the fundamental solution for Schrödinger equations, J. Fac. Sci. Univ. Tokyo IA 27 (1980), 193-226.

119. H. Kitada and H. Kumanoago, A family of Fourier integral operators and the fundamental solution for a Schrödinger equation, Osaka J. Math. 18 (1981), 291-360. 
120. V. Korotkov, Integral operators with Carleman kernels, Dokl. Akad. Nauk SSSR 165 (1965), 748-757.

121. S. Kotani, On asymptotic behavior of the spectra of a one dimensional Hamiltonian with a certain random coefficient, Publ. Res. Inst. Math. Sci. 12 (1976), 447-476.

122. V. F. Kovalenko, M. A. Perelmuter and Y. Semenov, Schrödinger operators with $L_{w}^{l / 2}\left(R^{l}\right)$ potentials, J. Math. Phys. 22 (1981), 1033-1044.

123. V. Kovalenko and Yu. Semenov, Some problems on expansions in generalized eigenfunctions of the Schrödinger operator with strongly singular potentials, Russian Math. Surveys 33 (1978), 119-157.

124. M. G. Krein, On the trace formula in the theory of perturbation, Mat. Sb. 33 (1953), 597-626.

125. , On perturbation determinants and a trace formula for unitary and selfadjoint operators, Dokl. Akad. Nauk SSSR 144 (1962), 268-271.

126. S. Kuroda, Scattering theory for differential operators. I, II, J. Math. Soc. Japan 25 (1973), 75-104; 222-234.

127. R. Lavine, The local spectral density and its classical limit (preprint).

128. H. Leinfelder, Gauge invariance of Schrödinger operators and related spectral properties, J. Operator Theory (to appear).

129. H. Leinfelder and C. G. Simader, Schrödinger operators with singular magnetic vector potentials, Math. Z. 176 (1981), 1-19.

130. E. Lieb and B. Simon, Pointwise bounds on eigenfunctions and wave packets in $N$-body quantum systems. VI. Asymptotics in the two cluster region, Adv. Appl. Math. 1 (1980), 324-343.

131. L. Lithner, A theorem of Phragmón Lindelof type for second order elliptic operators, Ark. Mat. 5 (1965), 281-285.

132. V. Maslov, On the asymptotics of generalized functions of the Schrödinger equation, Uspehi Mat. Nauk. 16 (1961), 253-254.

133. H. McKean, $-\Delta$ plus a bad potential, J. Math. Phys. 18 (1977), 1277-1279.

134. S. Mercuriev, On the asymptotic form of three body wave functions for the discrete spectrum, Soviet J. Nuclear Phys. 19 (1974), 222-229.

135. S. Molcanov, The structure of eigenfunctions of one dimensional unordered structures, Math. USSR-Isv. 12 (1978), 69-101.

136. J. Morgan, The exponential decay of subcontinuum wave functions of two electron atoms, J. Phys. A 10 (1977), 291.

137. J. Moser, On Harnack's theorem for elliptic differential equations, Comm. Pure Appl. Math. 14 (1961), 577-591.

138. W. Moss and J. Piepenbrink, Positive solutions of elliptic equations, Pacific J. Math. 75 (1978), 219-226.

139. C. Muller, On the behavior of the solution of the differential equation $\Delta u=f(x, u)$ in the neighborhood of a point, Comm. Pure Appl. Math. 1 (1954), 505-515.

140. S. Nakao, On the spectral distribution of the Schrödinger operator with a random potential, Japan J. Math. 3 (1977), 111-139.

141. E. Nelson, A quartic interaction in two dimension, Mathematical Theory of Elementary Particles, MIT Press, Cambridge, Mass., 1966, pp. 69-73.

142. _ The free Markov field, J. Funct. Anal. 12 (1973), 211-227.

143. A. O'Connor, Exponential decay of bound state wave functions, Comm. Math. Phys. 32 (1973), 319-340.

144. L. Pastur, On the Schrödinger equation with a random potential, Teoret. Mat. Fiz. 6 (1971), 415-424. (Russian)

145. On the distribution of the eigenvalues of the Schrödinger equation with a random potential, Funkcional. Anal. i Priložen. 6 (1972), 93-94. (Russian)

146. __ Spectrum of random selfadjoint operators, Uspehi. Mat. Nauk. 28 (1973), 1-64.

147. Behavior of some Wiener integrals as $t \rightarrow+\infty$ and the density of states of Schrödinger equation with a random potential, Teoret. Mat. Fiz. 32 (1977), 88-95. (Russian)

148. $\ldots$ Spectral properties of disordered systems in one-body approximation, Comm. Math. Phys. 75 (1980), 179.

149. D. Pearson, Singular measures in scattering theory, Comm. Math. Phys. 60 (1978), 13.

150. M. Perelmuter, Perturbation of operators with integral resolvent, Funkcional. Anal. i Priložen 14 (1980), 75. (Russian) 
151. P. Perry, I. Sigal and B. Simon, Spectral analysis of multiparticle Schrödinger operators, Ann. of Math. (2) 114 (1981), 519-567.

152. A. Persson, Bounds for the discrete part of the spectrum of a semibounded Schrödinger operator, Math. Scand. 8 (1960), 143-153.

153. J. Piepenbrink, Nonoscillatory elliptic equations, J. Differential Equations 15 (1974), 541-550.

154. _ A conjecture of Glazman, J. Differential Equations 24 (1977), 173-177.

155. S. Port and C. Stone, Brownian motion and classical potential theory, Academic Press, New York, 1978.

156. N. Portenko, Diffusion processes with unbounded drift coefficient, Theory Probab. Appl. 20 (1976), 27-37.

157. A. Povzner, On the expansion of arbitrary functions in terms of the eigenfunctions of the operator $-\Delta u+c u$, Mat. Sb. (N.S.) 32 (1953), 109-156. (Russian)

158. On eigenfunction expansions in terms of scattering solutions, Dokl. Akad. Nauk SSSR 104 (1955).

159. J. Rauch, private communication.

160. M. Reed and B. Simon, Methods of modern mathematical physics. I. Functional analysis, Academic Press, New York, 1972.

161. Methods of modern mathematical physics. II. Fourier analysis, self-adjointness, Academic Press, New York, 1975.

162. __ Methods of modern mathematical physics. IV. Analysis of operators, Academic Press, New York, 1978.

163. J. Rosen, Sobolev inequalities for weighted spaces and supercontractive estimates, Trans. Amer. Math. Soc. 222 (1976), 367-376.

164. O. Rothhaus, Lower bounds for eigenvalues of regular Sturm-Liouville operators and the logarithmic Sobolev inequality, Duke Math. J. 45 (1978), 351-362.

165. Logarithmic Sobolev inequalities and the spectrum of Sturm-Liouville operators, J. Funct. Anal. 39 (1980), 42-56.

166. Diffusion on compact Riemann manifolds and logarithmic Sobolev inequalities,

J. Funct. Anal. 42 (1981), 102-109.

167. Logarithmic Sobolev inequalities and the spectrum of Schrödinger operators, J. Funct. Anal. 42 (1981), 110-120.

168. Y. Saito, Eigenfunctions for the Schrödinger operators with long range potentials $Q(y)=$ $O\left(|y|^{-\varepsilon}\right)(\varepsilon>0)$, Osaka J. Math. 14 (1977), 11-35.

169. J. Saut and B. Scheurer, Un théorème de prolongement unique pour des opérateurs elliptiques dont les coefficients ne sont pas localement bornés, C. R. Acad. Sci. Paris Sér. A 290 (1980), 598-599.

170. M. Schechter, Hamiltonians for singular potentials, Indian Math. J. 22 (1972), 483-503.

171. __ Essential self-adjointness of the Schrödinger operator with magnetic vector potential,

J. Funct. Anal. 20 (1975), 93-104.

172. __ Spectra of partial differential operators, North-Holland, Amsterdam, 1971.

173. M. Schechter and B. Simon, Unique continuation for Schrödinger operators with unbounded potential, J. Math. Anal. Appl. 77 (1980), 482-492.

174. I. Sch'nol, On the behavior of the Schrödinger equation, Mat. Sb. 42 (1957), 273-286. (Russian)

175. I. Segal, Construction of nonlinear local quantum processes. I, Ann. of Math. (2) 92 (1970), 462-481.

176. , Construction of nonlinear local quantum processes. II, Invent. Math. 14 (1971), 211-241.

177. I. Sigal, Geometric parametrices in the $Q M N$-body problem, Duke Math. J. (to appear).

178. B. Simon, Essential self-adjointness of Schrödinger operators with positive potentials, Math. Ann. 201 (1973), 211-220.

179. S_ Schrödinger operators with singular magnetic vector potentials, Math. Z. 131 (1973), 361-370.

180. Pointwise bounds on eigenfunctions and wave packets in $N$-body quantum systems. I, Proc. Amer. Math. Soc. 42 (1974), 395-401.

181. Pointwise bounds on eigenfunctions and wave packets in $N$-body quantum systems. II, Proc. Amer. Math. Soc. 45 (1974), 454-456. 
182. Pointwise bounds on eigenfunctions and wave packets in $\mathrm{N}$-body quantum systems. III, Trans. Amer. Math. Soc. 208 (1975), 317-329.

183. A remark on Nelson's best hypercontractive estimates, Proc. Amer. Math. Soc. 55 (1976), 376-378.

184. An abstract Kato's inequality for generators of positivity preserving semigroups, Indian Math. J. 26 (1977), 1067-1073.

185. __ Kato's inequality and the comparison of semigroups, J. Funct. Anal. 32 (1979), 97-101.

186. Phase space analysis of simple scattering systems. Extensions of some work of Enss, Duke Math. J. 46 (1979), 119-168.

187. __ Maximal and minimal Schrödinger forms, J. Operator Theory 1 (1979), 37-47.

188. The classical limit of quantum partition functions, Comm. Math. Phys. 71 (1980), 247-276.

189. Brownian motion, $L^{p}$ properties of Schrödinger operators and the localization of binding, J. Funct. Anal. 35 (1980), 215-229.

190. (1981), 66-83.

191. _ Large time behavior of the $L^{p}$ norm of Schrödinger semigroups, J. Funct. Anal. 40 (1981), 66-83.

192. __ Almost periodic Schrödinger operators: A review, Adv. Appl. Math. (to appear).

193. The $P(\phi)_{2}$ Euclidean (quantum) field theory, Princeton Univ. Press, Princeton, N.J., 1974.

194. _ Functional integration and quantum physics, Academic Press, New York, 1979.

195. __ Trace ideals and their applications, Cambridge Univ. Press, London, 1979.

196. B. Simon and R. Hoegh-Krohn, Hypercontractive semigroups and two dimensional selfcoupled Bose fields, J. Funct. Anal. 9 (1972), 121-180.

197. E. Slaggie and E. Wichmann, Asymptotic properties of the wave functions for a bound non-relativistic system, J. Math. Phys. 3 (1962), 946-968.

198. I. M. Slivnyak, Spectrum of the Schrödinger operator with a random potential, Z. Vyčisl. Mat. i Mat. Fiz. 6 (1966), 1104-1108. (Russian)

199. G. Stampacchia, Le problem de Dirichlet par les equations elliptiques du second order á coefficients discontinue, Ann. Inst. Fourier (Grenoble) 15 (1969), 189-258.

200. F. Stummel, Singulare elliptische differentialoperatoren in Hilbertschen Räumen, Math. Ann. 132 (1956), 150-176.

201. W. Thirring, A course in mathematical physics. Vol. 4. Quantum mechanics of large systems, Springer-Verlag, Berlin and New York (to appear).

202. F. Trèves, Topological vector spaces, distributions and kernels, Academic Press, New York, 1967.

203. N. Trudinger, Linear elliptic operators with measurable coefficients, Ann. Scuola Norm. Sup. Pisa Sci. Fis. Mat. 27 (1973), 255-308.

204. G. Velo and A. S. Wightman (eds.), Constructive quantum field theory, Springer-Verlag, Berlin and New York, 1973.

205. F. Weissler, Logarithmic Sobolev inequalities and hypercontractive estimates on the circle,

J. Funct. Anal. 37 (1980), 218-234.

206. C. Wilcox, Theory of Bloch waves, J. Analyse Math. 33 (1978), 146-167.

207. S. Zelditch, Reconstruction of singularities for solutions of Schrödinger's equation, Comm. Math. Phys. (to appear).

Departments of Mathematics and Physics, California Institute of Technology, Pasadena, California 91125 